\title{
Essays on pricing electricity and electricity derivatives in deregulated markets
}

Julia Popova

West Virginia University

Follow this and additional works at: https://researchrepository.wvu.edu/etd

\section{Recommended Citation}

Popova, Julia, "Essays on pricing electricity and electricity derivatives in deregulated markets" (2008). Graduate Theses, Dissertations, and Problem Reports. 2694.

https://researchrepository.wvu.edu/etd/2694

This Dissertation is protected by copyright and/or related rights. It has been brought to you by the The Research Repository @ WVU with permission from the rights-holder(s). You are free to use this Dissertation in any way that is permitted by the copyright and related rights legislation that applies to your use. For other uses you must obtain permission from the rights-holder(s) directly, unless additional rights are indicated by a Creative Commons license in the record and/ or on the work itself. This Dissertation has been accepted for inclusion in WVU Graduate Theses, Dissertations, and Problem Reports collection by an authorized administrator of The Research Repository @ WVU.

For more information, please contact researchrepository@mail.wvu.edu. 


\title{
ESSAYS ON PRICING ELECTRICITY AND ELECTRICITY DERIVATIVES IN DEREGULATED MARKETS
}

\author{
Julia Popova
}
Dissertation submitted to the College of Business and Economics at West Virginia University in partial fulfillment of the requirements for the degree of Doctor of Philosophy
in
Economics

Stratford M. Douglas, Ph.D., Chair

Alexei Egorov, Ph.D.

Jerald J. Fletcher, Ph.D.

Hyungna Oh, Ph.D.

Timothy T. Phipps, Ph.D.

Department of Economics

Morgantown, West Virginia 2008

Keywords: Electricity Prices, Electricity Markets, Forward Premium, Spatial Econometrics, Market Efficiency, Stationarity

Copyright 2008 Julia Popova 


\title{
Abstract \\ ESSAYS ON PRICING ELECTRICITY AND ELECTRICITY DERIVATIVES IN DEREGULATED MARKETS
}

\begin{abstract}
Julia Popova
This dissertation is composed of four essays on the behavior of wholesale electricity prices and their derivatives. The first essay provides an empirical model that takes into account the spatial features of a transmission network on the electricity market. The spatial structure of the transmission grid plays a key role in determining electricity prices, but it has not been incorporated into previous empirical models. The econometric model in this essay incorporates a simple representation of the transmission system into a spatial panel data model of electricity prices, and also accounts for the effect of dynamic transmission system constraints on electricity market integration. Empirical results using PJM data confirm the existence of spatial patterns in electricity prices and show that spatial correlation diminishes as transmission lines become more congested. The second essay develops and empirically tests a model of the influence of natural gas storage inventories on the electricity forward premium. I link a model of the effect of gas storage constraints on the higher moments of the distribution of electricity prices to a model of the effect of those moments on the forward premium. Empirical results using PJM data support the model's predictions that gas storage inventories sharply reduce the electricity forward premium when demand for electricity is high and space-heating demand for gas is low. The third essay examines the efficiency of PJM electricity markets. A market is efficient if prices reflect all relevant information, so that prices follow a random walk. The hypothesis of random walk is examined using empirical tests, including the Portmanteau, Augmented Dickey-Fuller, KPSS, and multiple variance ratio tests. The results are mixed though evidence of some level of market efficiency is found. The last essay investigates the possibility that previous researchers have drawn spurious conclusions based on classical unit root tests incorrectly applied to wholesale electricity prices. It is well known that electricity prices exhibit both cyclicity and high volatility which varies through time. Results indicate that heterogeneity in unconditional variance - which is not detected by classical unit root tests - may contribute to the appearance of non-stationarity.
\end{abstract}




\section{Acknowledgments}

I would like to express my gratitude to the following people for their support and assistance in completing this dissertation. First I would like to thank all the members of my dissertation committee: Stratford M. Douglas, Alexei Egorov, Hyungna Oh, Jerald Fletcher, and Timothy Phipps. Each person has been extremely helpful at various stages of my research. Their insights and support have been invaluable and I am honored to have every one of them on my committee. I especially would like to thank Dr. Douglas who provided numerous hours of his time over the past five years to me in the form of course work, appointments, proofreading, and support. I will be grateful forever for his advice and patience. I have also been fortunate to work with excellent colleagues in graduate school, so, I would like to express my gratitude to Serhiy Kotsan, Sudeshna Pal, Justin Ross, and Daniel Suh who have helped me significantly to go all through the process. 


\section{Table of Contents}

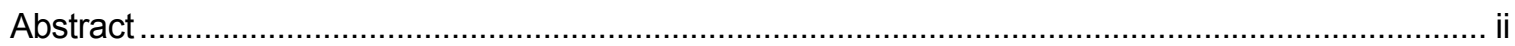

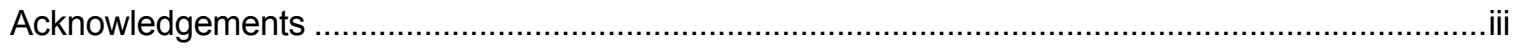

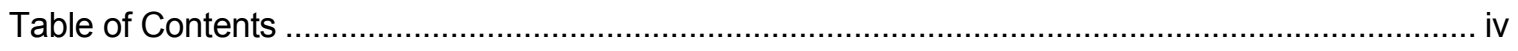

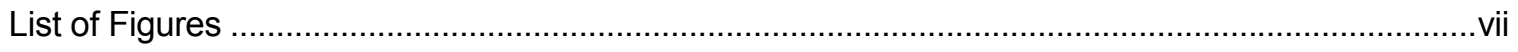

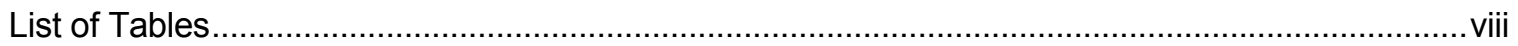

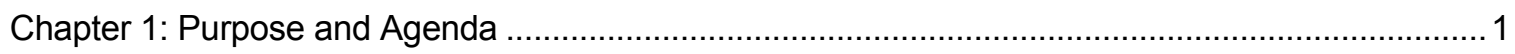

Chapter 2: Econometric Estimation of Spatial Patterns in Electricity Prices ...................................... 5

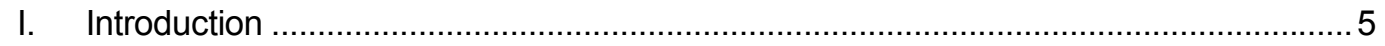

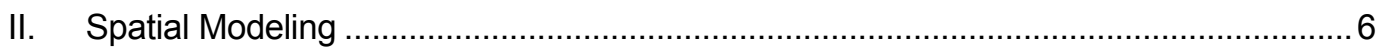

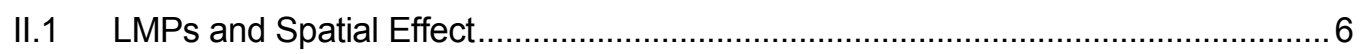

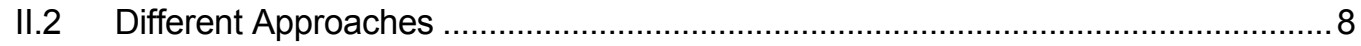

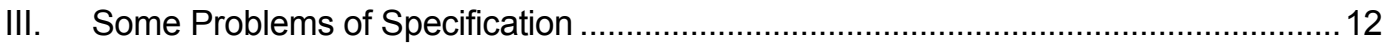

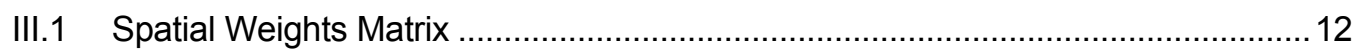

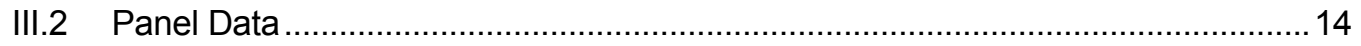

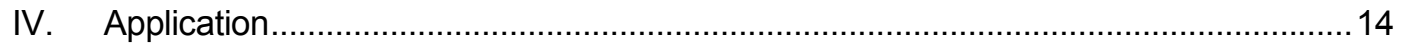

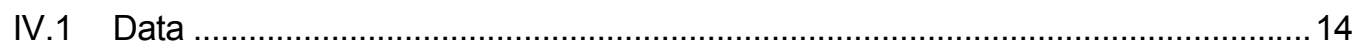

IV.2 Electricity Price Characteristics..................................................................... 16

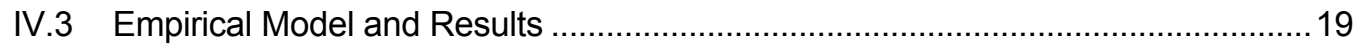

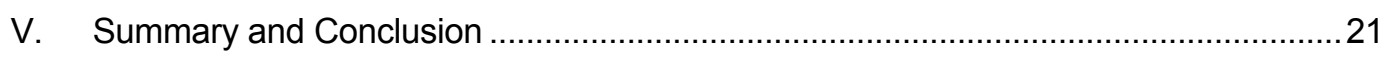

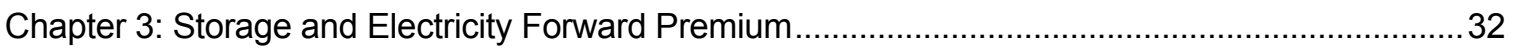

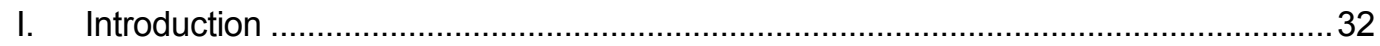

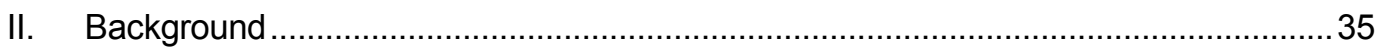

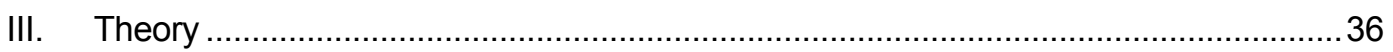

III.1 How the Distribution of Electricity Spot Prices Affects the Future

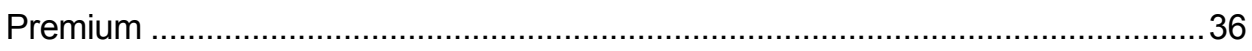


III.2 How Gas Storage Inventories Affect the Distribution of Electricity

Prices

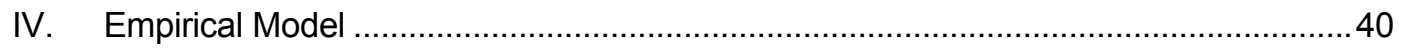

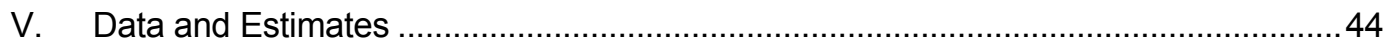

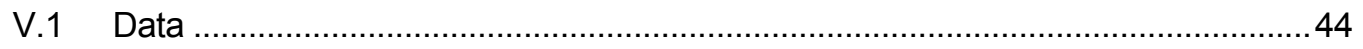

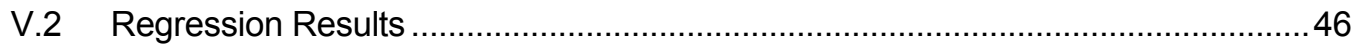

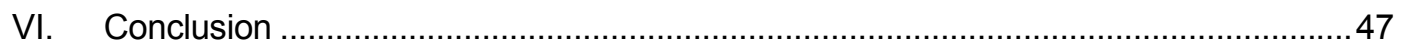

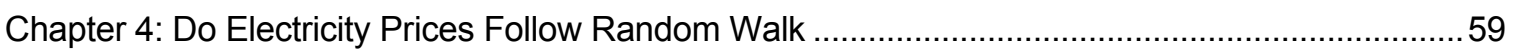

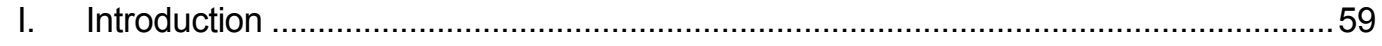

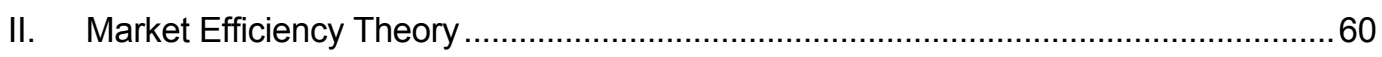

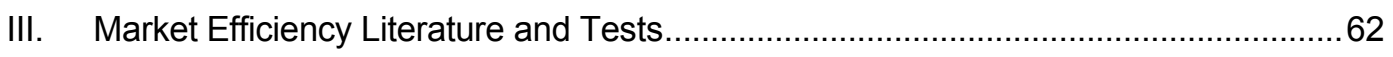

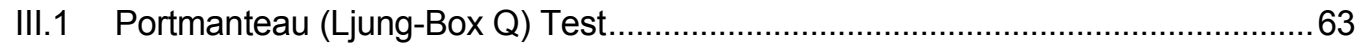

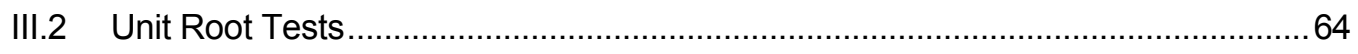

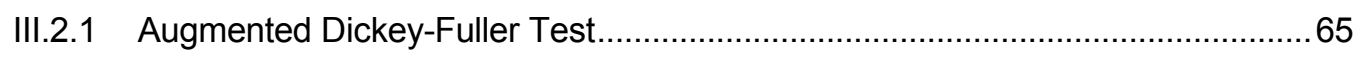

III.2.2 Dickey-Fuller Generalized Least Squares (DF-GLS) test..................................66

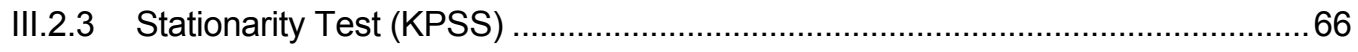

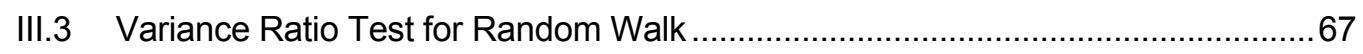

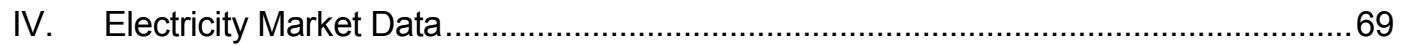

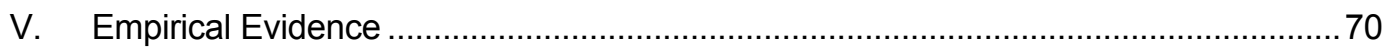

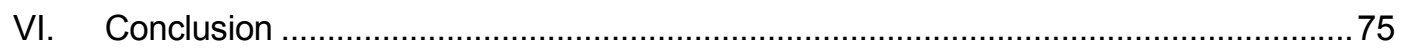

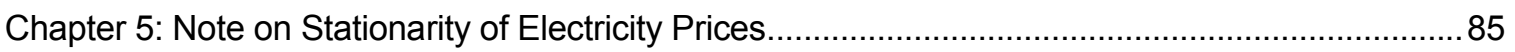

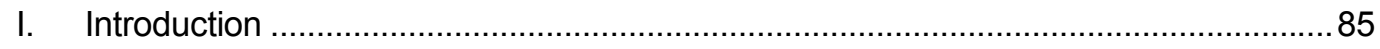

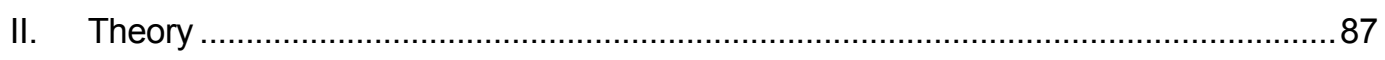

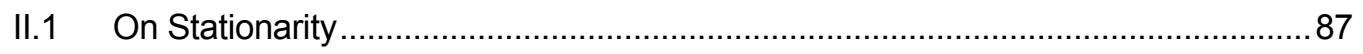

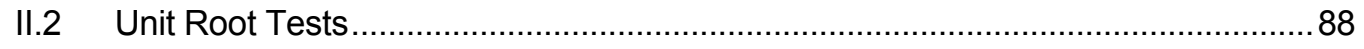

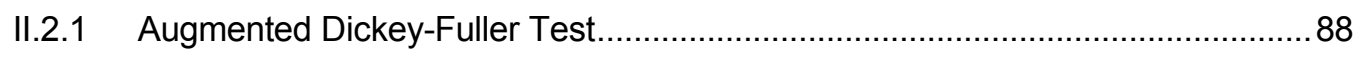

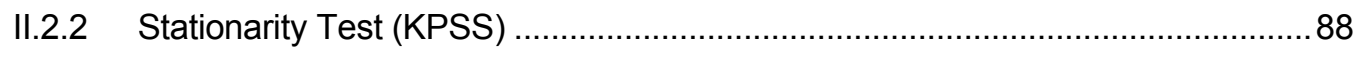

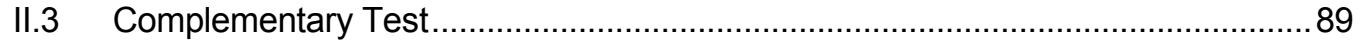


III. Electricity Market Data Description .................................................................. 90

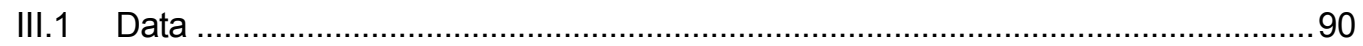

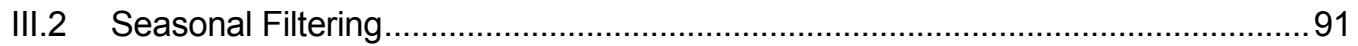

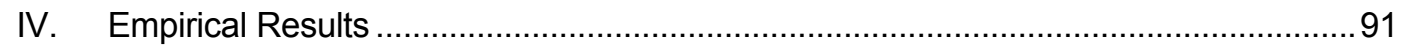

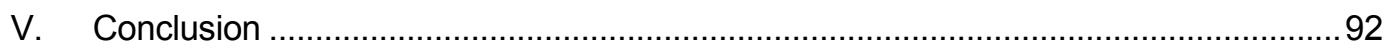

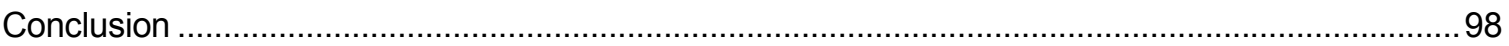

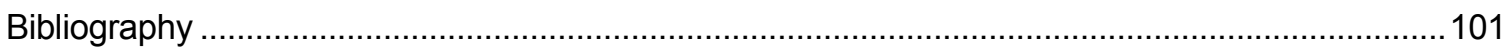

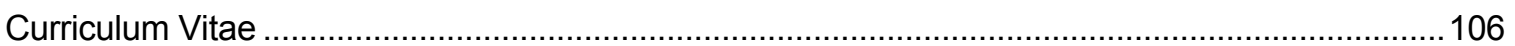




\section{List of Figures}

Figure 2-1: Seasonal Cycles for Real-Time and Day-Ahead Prices ................................................ 30

Figure 2-2: Estimated Spatial Coefficient for Electrical Contiguity Weight Matrix ............................. 31

Figure 2-3: Estimated Spatial Coefficient $\quad$ for Geographical Contiguity Weight Matrix ....................... 31

Figure 3-1: Simulated Effect of Natural Gas Availability on Electricity Price Skewness ....................... 52

Figure 3-2: Estimated Effects of Recent Spot Price Skewness, and Variance on the Electricity

Forward Premium, with 95\% Confidence Intervals ................................................................ 53

Figure 3-3: Estimated Marginal Effect of Temperature on the Forward Premium .............................. 54 


\section{List of Tables}

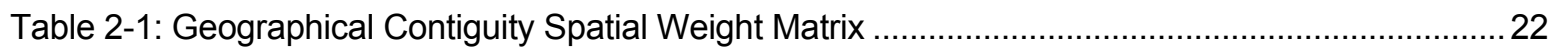

Table 2-2: Electrical Contiguity Spatial Weight Matrix ................................................................... 22

Table 2-3: Number of Constraint Contingencies by Time of a Day ................................................... 23

Table 2-4: Summary Statistics for Hourly Electricity Prices ……...................................................... 24

Table 2-5: Summary Statistics by Number of Constraints for Spot and Day-Ahead Prices Averaged

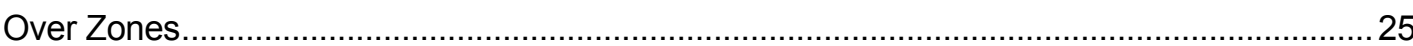

Table 2-6: Summary Statistics by Number of Constraints for Spot and Day-Ahead Prices Averaged

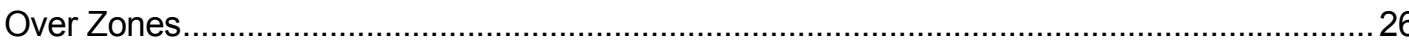

Table 2-7: Spatial Panel Econometric Estimation Results, Geographical Contiguity Weight Matrix

Random Effects Panel Estimation Dependent Variable: Spot Price .......................................2 27

Table 2-8: Spatial Panel Econometric Estimation Results, Electrical Contiguity Weight Matrix

Random Effects Panel Estimation Dependent Variable: Spot Price ....................................... 28

Table 2-9: Regression Results, Panel Data Random Effects (Non-Spatial Pooled Data) Dependent

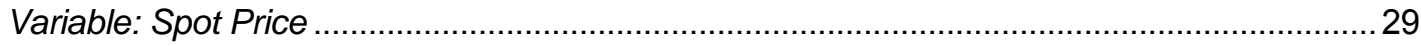

Table 3-1 Predicted Effects of Variables on Electricity Price Moments and the Forward Premium ...... 40

Table 3-2: Summary Statistics for PJM Real-Time Spot Electricity Price ...........................................49

Table 3-3: Summary Statistics for PJM Day-Ahead Forward Price.................................................... 50

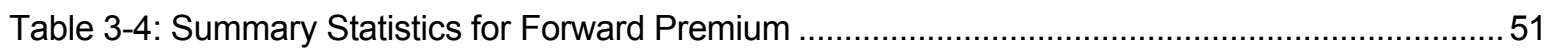

Table 3-5: Regression Results. Dependent Variable: Forward Premium ............................................55

Table 3-6: Regression Results. Dependent Variable: Forward Premium .........................................56

Table 3-7: Regression Results. Dependent Variable: Forward Premium ..........................................57

Table 3-8: Regression Results. Dependent Variable: Forward Premium .......................................... 58

Table 4-1: Summary Statistics for Real-Time Prices .................................................................... 77

Table 4-2: Summary Statistics for Day-Ahead Price ................................................................... 78 
Table 4-3: Ljung-Box (White Noise) Q-statistics for Day-Ahead Electricity Prices Returns. 79

Table 4-4: Ljung-Box (White Noise) Q-statistics for Real-Time Electricity Prices Returns. 80

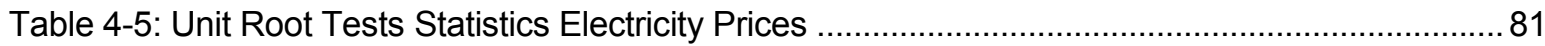

Table 4-6: Summary of Unit Root Tests Results for Electricity Prices.............................................. 82

Table 4-7: Multivariate Variance Ratio Test for Real-Time Prices...................................................... 83

Table 4-8: Multiple Variance Ratio Test for Day-Ahead Prices. ....................................................... 84

Table 5-1: Summary Statistics for Real-Time Prices ....................................................................... 94

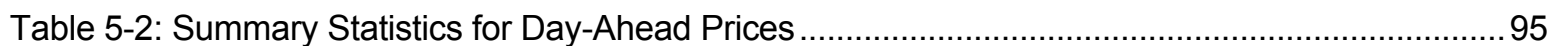

Table 5-3: ADF Level Unit Root and KPSS Level Stationarity Tests Results ....................................96

Table 5-4: Complementary Test for Stationarity for Electricity Prices …..........................................97 


\section{Chapter 1 : Purpose and Agenda}

Electricity is not a typical commodity since it must be produced at the same time it is consumed. Non-storability, inflexible supply, fluctuating and inelastic demand, transmission congestion and generation capacity issues - all these features of deregulated electricity markets distinguish them from other commodity and financial markets. To run a successful business in the deregulated power markets requires correct understanding of electricity prices evolution, behavior, and dynamics. This understanding is a key for analyzing investment and production decisions, building efficient hedging instruments and other financial products. The objective of this dissertation is; (1) to examine properties of electricity prices, (2) to investigate effect of transmission network structure and its congestion level on electricity prices formation; (3) to analyze the role of fuel storage inventories in mitigating economic risk in forward trading, (4) and to study the structure of forward and spot markets and evaluate degree of informational efficiency.

Chapter 2 utilizes spatial econometrics panel tools to take into account the features of a transmission lines network structure as well as estimate the effect of transmission lines congestion on electricity price setting ${ }^{1}$. In an electricity market, the pattern of prices in any location is strongly affected by the pattern of prices in other locations. The instantaneous and pervasive effects of transmission lines capacity constraints create unique challenges for modeling electricity prices relative to prices of other commodities. When transmission lines are congested, the energy from one area cannot be freely transported to another area. In this situation, prices in the two areas diverge and set based on marginal costs of production an additional unit of electricity in each location. Consequently, there are two risk components imbedded into the electricity prices. One comes from the basic pricing methodology in case of no congestion in the system, and another comes from the fact that congestion costs must be borne by producers. Therefore, the spatial structure of the market play important role in pricing the electricity and should be explicitly included

\footnotetext{
${ }^{1}$ This paper is under second revision in the Energy Journal. Dr. Douglas is the second author and he agreed that the paper could be included into my dissertation
} 
into the model This suggests that a spatial autoregressive in errors model - which incorporates the effect of space - may be appropriate for modeling prices. To our knowledge, no previous research has attempted to use the spatial econometrics techniques for electricity prices modeling, though they widely utilized in public economics, public finance, and regional economics. The model adds to the existing literature on electricity price study by analyzing transmission congestion and spatial correlation in addition to sounded electricity price characteristics such as seasonal fluctuations and volatility. The model is applied to equilibrium electricity spot prices of the Pennsylvania New-Jersey and Maryland (PJM) market. We show that spatial correlation between zonal prices is significant and diminishes as the system becomes bound by transmission lines congestion. Our empirical analysis indicates that both the constitution of the transmission lines network and the structure of the market are responsible for the spatial correlation in equilibrium electricity prices, which should not be ignored by careful research.

In Chapter 3, entitled "Storage and the Electricity Forward Premium," Stratford Douglas and I present an empirical model to study the effect of fuel storage on the electricity day-ahead forward premium $^{2}$. In perfectly competitive market at equilibrium forward premium should be non-significant on average. Due to the fact that electricity cannot be economically stored, classical theories of pricing forward contracts are not appropriate. Instead, the current theory of electricity forward contract pricing relies on a model of the supply and demand of hedges by risk-averse electricity producers and retail load serving entities (Bessembinder and Lemmon (2002)). Since power plant fuels may be stored (and power plant capacity reserved) arbitrage and conversion opportunities may still place limits upon electricity forward prices. The model constructed in this Chapter 3 , connects the effect of natural gas storage to the higher moments of the distribution of electricity prices and existing models of those moments on the forward premium in electricity markets. The main prediction is a sharply negative effect of gas storage on the electricity forward premium when electricity loads are high and demand for natural gas is low; but negative or insignificant when demand for electricity is light. Empirical results, based on hourly electricity price data from the PJM 
markets and weekly gas storage data from the Energy Information Agency, strongly support the model. The model built the paper links two theoretical models of Routledge et al (2001) and Bessembinder and Lemmon (2002). Empirical evidence supports Bessembinder and Lemmon's predictions in part while strongly confirms the Routledge et al theory.

In Chapter 4, I present thorough discussion on outlining and testing for electricity markets efficiency. A market is said to be informational efficient if it fully and correctly reflects all relevant information in setting prices. Following Roberts (1967) and Campbell et al (1997), an "efficient market" hypothesis is set as a hypothesis of whether equilibrium prices cab be represented by random walk. There is a number of econometric techniques developed to test for random walk. Among the most utilized are classical unit root test such as Augmented Dickey-Fuller (ADF) test and its numerous modifications, as well as Kwiatkowski, Phillips, Schmidt, and Shin (KPSS) stationarity test. These tests produce opposite results for some hours. Additional discussion on this effect is provided in the Chapter. Although theoretical distributions of both tests do not change when seasonal dummies included, both ADF and KPSS may produce spurious results due to ignored seasonal cycles, which are sounded characteristics of electricity prices. In addition I use random walk multiple variance ratio test developed by Lo and MacKinlay (1988), Chow and Denning (1993) to show that on-peak-hours electricity prices may be called random walk. In general, empirical results are mixed though evidence of some level of market efficiency is found.

The fourth essay, presented in Chapter 5, comments on bogus conclusions that may be drawn based on classical unit root tests applied to wholesale electricity prices. Using theory and following reasoning of Ahamada (2004) and Liew et al (2007), we show that heterogeneity in unconditional variance - while failed to be detected by classical unit root tests - may be a big contributor to non-stationarity in electricity prices. This Chapter comments on the fact that even seasonal fluctuations free, electricity prices are not stationary. Therefore, caution should be taken in using autoregressive time series techniques for modeling and forecasting.

2 This paper is published in Energy Economics, 30(4), Dr. Douglas is the first author and he agreed that the paper could be included in my dissertation 
This dissertation concludes Chapter 6 with a summary of major results from each of the essays. Furthermore, a discussion of possible future research is provided. 


\section{Chapter 2 : Econometric Estimation of Spatial Patterns in Electricity Prices}

\section{Introduction}

The electricity transmission system provides the framework for the constantly changing spatial pattern of prices in modern wholesale electricity markets. Locational marginal-cost prices (LMPs) in those markets reflect the full marginal cost of power delivery at each time and place, and the physical characteristics of transmission lines and the constantly changing state of transmission system constraints play a key role in determining their level and volatility, as discussed by Bohn, Caramanis, and Schweppe (1984) and Stoft (2002), inter alia. The importance of the transmission grid's structure in determining efficiency, reliability, and prices in electricity markets is emphasized in the electricity market literature, including Borenstein (2002), Borenstein, Bushnell, and Wolak (2002), De Vany and Walls (1999b), Hogan (1992, 1993), Jerko, Mjelde, and Bessler (2004), and Lucia and Schwartz (2002). Although transmission system conditions strongly influence the level and volatility of electricity prices, the transmission system generally has not been modeled in previous econometric studies of electric power markets. Standard time series and panel data econometric tools most commonly used by energy modelers can not readily estimate or control for the effects of spatial market structure. The adverse effects of ignoring spatial correlation on modeling and forecasting spatially correlated variables are well known, however, as discussed in Giacomini and Granger (2004).

The objective of this study is to introduce the use of spatial econometric tools to characterize and control for the effect of transmission network structure and conditions on electricity price dynamics. Previous researchers have recognized the importance of spatial relationships in determining electricity price levels and volatility. For example, Park, Mjelde, and Bessler (2006) look at econometric relationships among the spot markets in different parts of the United States, and find that transmission interconnections appear to drive contemporaneous correlations among 
markets and local price innovations have global effects. Worthington, Kay-Spratley, and Higgs (2005) emphasize the importance of the physical network between markets for information spillover and integration. Borenstein (2002) argues that transmission congestion is the primary reason for volatile price behavior.

Our model provides a first demonstration of how to conceive and construct a spatial econometric model that takes into account physical attributes of the transmission system. We use data from the PJM Interconnection from April 2002 to May 2006, and estimate the spatial autocorrelation among the twelve PJM regions that existed at that time. We also incorporate into our analysis real-time data from PJM on constraint contingencies for each hour, and allow for the transmission grid topology to affect formation of market prices. Our methods detect the effect of spatial influences in electricity price dynamics, and we argue that ignoring the spatial characteristics of the market and transmission network structure results in biased and inefficient estimates. We do not intend the models presented here to be the final word in spatial electricity price models; rather, we intend simply to demonstrate a way of thinking about modeling spatial structure that researchers and practitioners can build upon and modify to suit their own disparate modeling objectives. In particular, investigators with more detailed proprietary information about the real-time state of the transmission system information should be able to adapt our model to use that information to improve forecasts and empirical insights into the functioning of electricity markets.

This paper has five sections. Following this introductory section we discuss the origin of spatial patterns and explore alternative approaches for modeling the spatial structure of wholesale electricity prices. In section 3 we discuss econometric model specification problems including the structure of the spatial weights matrix, and dealing with non-normality, seasonality, and the panel aspect of electricity price data. In section 4 we discuss our data set and our empirical model, and then present and analyze the results of our estimation example. Section 5 concludes.

\section{Spatial Modeling}

\section{II.1 LMPs and Spatial Effects}


The PJM Interconnection, New York ISO, ISO New England, Texas, Australia, and most other modern electricity markets use a Locational Marginal Cost Price (LMP) methodology to set electricity prices at different nodes throughout the system. The conceptual basis for calculation of LMPs is explained in Bohn, Caramanis, and Schweppe (1984), and further developed and explained by many others in the trade press and academic literature, notably Hogan $(1992,1993)$ and Stoft (2002). Viewing the efficient operation of the electrical power system as a constrained maximization problem, Bohn, Caramanis, and Schweppe showed that the efficient price at any given node is the opportunity cost of producing and delivering an additional unit (MW) of power at that node. The efficient LMP then is the marginal running cost of the marginal plant, plus additional line losses associated with the delivery of additional power to the node in question, plus the shadow value of any constraints on the system, as represented by Lagrange multipliers associated with transmission and generation constraints. The PJM system's implementation of LMPs ignores line losses, and focuses on marginal plant operating costs plus costs associated with transmission line congestion.

The North American electrical grid is a vast unified network of synchronized generators and random consumers, sometimes referred to as the "world's biggest machine." A change in electricity injection or withdrawal at any node will in principle affect LMPs at every other node on the network. A change at any node will affect flows on all transmission lines through Kirchhoff's Laws, which will change line losses, and may cause one or more lines to reach its voltage or thermal constraint. If there are no transmission constraints and line losses are ignored, the LMP will be the same at all nodes and a price change at one node will instantaneously propagate to all other nodes. The effect is generally more noticeable for large and nearby changes, and the effect of a small change (such as switching on a single light bulb) may be imperceptible, but in principle any change at any node affects all nodes, and the effects propagate virtually instantaneously. If there are transmission constraints then prices will diverge at all points in amounts determined by the extent to which delivery to and from that node is affected by the constraint. Price will decline at nodes where 
additional consumption will help relieve the constraint, and price will rise at nodes where additional production will help relieve the constraint.

Thus, there are three key factors that should be taken into account in any spatial model of electricity price formation. First, disturbances to prices at one point affect prices at all points; that is, the correlation among prices is global. Second, the correlation of prices is mediated by the transmission system, so any model of the spatial structure of electricity prices must reflect in some way the topology of the transmission system. Third, the relationships change among prices at different points in the system when the system becomes constrained, and the details and extent of the constraints matter.

\section{II.2 Different Approaches}

The ideal spatial model of power prices would contain within it an engineering model of the transmission system whose topology would change as the spatial pattern of injections and withdrawals change and as contingencies occur. In short, the ideal model would be the same as the model used by the engineers operating the system and it would have the same voracious appetite for detailed real time information. Our purpose here is to explore a framework for modeling the spatial structure of prices that has fewer informational requirements, one that uses spatial information without requiring the full dynamic information set available to the system operator, but that can be adapted in appropriate ways by modelers with different information sets.

Spatial econometrics provides a promising set of tools for this task. As a field it began with the work of Cliff and Ord (1973), Ord (1975), and Paelinck and Klaassen (1979), and it has benefited in recent years from cross-fertilization and cooperation among researchers in Economics,

Geography, and Regional Science. Spatial econometric applications and theory have increasingly appeared in mainstream economics journals in recent years, e.g. Kelejian and Prucha $(1998,1999)$, Baltagi and Li (2001), LeSage and Pace (2004, 2007), and Kapoor, Kelejian, and Prucha (2007). Anselin (1988) is a standard work and provides a summary and analysis of the subject up to that date, while Anselin (2002) provides a useful taxonomy of spatial econometric models. LeSage 
(1999) provides further practical and theoretical information, along with the extensive set of welldesigned MatLab programs. A special issue of the Journal of Econometrics (Vol. 140, Issue 1) devoted to spatial econometrics appeared in October 2007.

An engineering-style representation of the spatial relationships between electricity prices in $\mathrm{N}$ different locations would constitute what Anselin (2002) refers to as a "direct" approach to spatial model specification, in which the full set of $N(N-1)$ spatial relationships is explicitly modeled. For example, Dubin (1988) uses an $N$ by $N$ matrix that specifies the correlations between each pair of observations. Much more common in the spatial literature, however, is the "indirect" approach, in which each relationship between neighbors is specified explicitly (often simply as a 0,1 indicator of contiguity) in a spatial weights matrix, $\mathrm{W}$, generally in combination with a scalar decay parameter $\rho$. The relationships among non-neighbors follow naturally as powers of $\rho \mathrm{W}$ : neighbors of neighbors are related as $\rho^{2} W^{2}$, and so on. Although $W$ is typically a sparse matrix, with $W_{i, j} \neq 0$ only if $i$ and $j$ are neighbors, higher powers of $\mathrm{W}$ become increasingly dense.

Specifically, given the standard linear regression model with panel data and borrowing notation from Kapoor, Kelejian, and Prucha (2007),

$$
y_{N}(t)=x_{N}(t) \beta+\varepsilon_{N}(t)
$$

where $\mathrm{y}_{N}(\mathrm{t})$ is an $N$ by 1 vector of prices observed at time $t, \mathrm{x}_{N}(\mathrm{t})$ is the $N$ by $k$ matrix of exogenous variables at time t and $\varepsilon_{N}(\mathrm{t})$ is the corresponding $N$ by 1 vector of errors. In a first order spatial autoregressive (SAR) error model, the error term in location $i$ is contemporaneously correlated with its neighbors, as specified in the $N$ by $N$ spatial weights matrix W. In its "reduced form" the SAR error vector

$$
\varepsilon_{N}(t)=\rho W \varepsilon_{N}(t)+u_{N}(t)
$$

contains a disturbance vector $\mathrm{u}$ (t) and the spatial autoregressive intensity parameter $\rho$, which is a scalar that measures the overall degree of spatial dependence in the system. To allow for cross time correlation Kapoor, Kelejian, and Prucha specify an error components model for u: 


$$
u_{N}(t)=\mu_{N}+v_{N}(t)
$$

where the $N$ by 1 vector $\mu_{\mathrm{N}}$ is a location-specific error component vector, components of which identically distributed with zero mean and variance $\sigma_{\mu}{ }^{2}$. The disturbance $v_{N}(t)$ is usually assumed to be normally distributed with zero mean and variance $\sigma_{v}{ }^{2}$, though normality is not required for consistency of GMM estimators. Following Anselin (2003), manipulate equation (2) to obtain

$$
\varepsilon_{N}(t)=(I-\rho W)^{-1} u_{N}(t)
$$

where I is the $N$ by $N$ identity matrix. Note that if $|\rho|<1$ using a Leontief expansion $(I-\rho W$

$$
\begin{array}{r}
)^{-1}=I+\sum_{j=1}^{\infty}(\rho W)^{j} \text {, so }(3) \text { can be rewritten as } \\
\varepsilon_{\mathrm{N}}(\mathrm{t})=\left(I+\rho \mathrm{W}+\rho^{2} \mathrm{~W}^{2}+\ldots\right) \mathrm{u}_{\mathrm{N}}(\mathrm{t}),
\end{array}
$$

which implies that the effect of a disturbance in any particular region propagates instantaneously throughout all regions in a manner that is mediated through the structure of the spatial weight matrix $\mathrm{W}$, and diminishes with distance at a rate determined by the magnitude of $\rho$. The transmission of the disturbance occurs first through the nearest neighboring regions $(\rho \mathrm{W})$, then through the neighbors of the neighbors, $\left(\rho^{2} W^{2}\right)$, and so on throughout the system. The variancecovariance matrix $\mathrm{E}\left(\varepsilon \varepsilon^{\prime}\right)$, then becomes

$$
\begin{aligned}
& \mathrm{E}\left(\varepsilon_{\mathrm{N}}(\mathrm{t}) \varepsilon_{\mathrm{N}}(\mathrm{t})^{\prime}\right)=\left(\sigma_{\mu}{ }^{2}+\sigma_{v}{ }^{2}\right)(\mathrm{I}-\rho \mathrm{W})^{-1}\left(\mathrm{I}-\rho \mathrm{W}^{\prime}\right)^{-1^{\prime}}= \\
& =\left(\sigma_{\mu}{ }^{2}+\sigma_{v}{ }^{2}\right)\left[\mathrm{I}+\rho\left(\mathrm{W}+\mathrm{W}^{\prime}\right)+\rho^{2}\left(\mathrm{~W}^{2}+\mathrm{WW}^{\prime}+\mathrm{W}^{{ }^{2}}\right)+\ldots\right]
\end{aligned}
$$

which means that the error terms in all regions are correlated with each other, but nearer neighbors are more correlated than more distant neighbors, so long as $|\rho|<1$. Thus, in the context of electricity prices, the effect of a disturbance anywhere on the grid is instantaneous, global, and dependent on the spatial structure of the network, as it should be. The rate of decay of the correlation with distance is driven by the parameter $\rho$; thus, for a well specified model of an 
unconstrained electrical grid we would expect $\rho$ to be only slightly less than 1 . As the severity of transmission constraints increases, however, we would expect $\rho$ to fall.

The SAR error model (2) implies a specific structural relationship among prices and their determinants across space that mirrors the relationship among the errors. To see this relationship, substitute (3) into (1), premultiply by $(I-\rho W)$, and rearrange terms to obtain the "structural form" expression for the SAR error model:

$$
y_{N}(t)=\rho W y_{N}(t)+(I-\rho W) x_{N}(t) \beta+u_{N}(t)
$$

which again emphasizes the "global" nature of the SAR errors model. The model can also be written for a particular observation of the price $y_{t i}$ at location $i$ and time $t$ as

$$
y_{t i}=\rho W_{i} y_{N}(t)+\left(I_{i} \cdot-\rho W_{i} \cdot\right) x_{N}(t) \beta+u_{t i}
$$

where $\rho, y_{t i}$, and $u_{t i}$ are scalars, and $\mathrm{I}_{i}$, and $\mathrm{W}_{i}$ are the $i$ th rows of the identity matrix and the spatial weights matrix $W$ respectively. As in $(5), y_{N}(t)$ is the $N$ by 1 vector of prices at time $t, x_{N}(t)$ is the $N$ by $k$ matrix of exogenous variables at time $t$, and $\mathrm{l}_{i} \cdot \mathrm{x}_{\mathrm{N}}(\mathrm{t})=\mathrm{x}_{\mathrm{N}}(\mathrm{t})_{i}$ is the $i$ th row of $\mathrm{x}_{\mathrm{N}}(\mathrm{t})$, which is the set of $\mathrm{k}$ exogenous variables that obtain at time $t$ and location $i$.

In other words, equations (5) and (5') demonstrate that the SAR model, despite the apparent limitation of the spatial correlation to the error term, relates the price $\left(y_{t i}\right)$ at each node in the network to prices in all neighboring nodes $\left(\rho \mathrm{W}_{i \cdot} \mathrm{y}_{\mathrm{N}}(\mathrm{t})\right)$ and conditions at all neighboring nodes $\left(\rho W_{i} \cdot x_{N}(t) \beta\right)$, as well as the observed and unobserved conditions at that node $\left(\mathrm{x}_{\mathrm{N}}(\mathrm{t})_{i} \cdot \beta+\mathrm{u}_{t i .}\right)$. This global model of price formation is consistent with the pervasive spatial correlation specified in Bohn, Caramanis, and Schweppe (1984), and in the calculations used by PJM and other systems in formulating LMP electricity prices.

An alternative approach is the SAR in variables model:

$$
\mathrm{y}_{N}(\mathrm{t})=\lambda W \mathrm{y}_{\mathrm{N}}(\mathrm{t})+\mathrm{x}_{\mathrm{N}}(\mathrm{t}) \beta+\varepsilon_{\mathrm{N}}(\mathrm{t})
$$

in which the error term may or may not follow an SAR process as well. Because of the bidirectionality of spatial correlation, if the estimation technique ignores the spatial correlation then 
the OLS estimator of $\beta$ in (6) is biased and inconsistent, which would imply that all of the previous econometric literature on electricity prices is fatally flawed (Anselin, 1988). Testing for SAR in variables is therefore a matter of considerable interest. As the spatial literature does not offer any appropriate tests in a panel data context, and development of such tests is beyond the scope of the present work, we defer addressing this question and follow the mainstream of the applied spatial econometric literature in using the SAR in errors model as expressed in (1) and (2).

\section{Some Problems of Specification}

Because of electricity's inelastic and constantly fluctuating demand, non-storability, and unique infrastructure, econometric modeling of wholesale electricity price data presents unique problems. Empirical distributions of electricity prices typically exhibit seasonality at daily, weekly, and yearly frequencies, mean reversion, volatility clustering, positive skewness, and excess kurtosis. Several papers have acknowledged the importance of transmission system congestion, but without employing spatial analysis. Hadsell et al. (2004), for instance, estimates conditional volatility in five deregulated electricity markets of the US employing GARCH models, stressing the importance of transmission system congestion in the generation of electricity price series. Similarly Borenstein (2002) emphasizes the key role of congestion and capacity constraints, as well as demand inelasticity and electricity non-storability as causes of severe volatility in electricity prices.

\section{III.1 Spatial Weights Matrix}

The spatial weight matrix $\mathrm{W}$ expresses the geographic structure of economic interactions between regions, which in our case are the twelve zones of the PJM interconnection during the time period covered by our dataset. Ideally, the scalar $\rho W_{i} \cdot \varepsilon_{N}(t)$ is a weighted sum of all of the errors in the system at time $t$, with the weights $W_{j i}$ corresponding to the amount of influence of the error $\varepsilon$ in node $\mathrm{j}$ on the error in node $i$. In the applied spatial econometrics literature the weights are commonly formulated as dummy variables indicating geographical contiguity, often normalized according to the number of contiguous regions. Two regions are defined as being geographically contiguous if they have a common border of non-zero length. Thus, if region $j$ is one of four regions 
that shares a common border with region $i$, then the $(i, j)$ element of $W$ would be $(1 / 4)$ in the normalized formulation. Such a geographical contiguity weight matrix is of questionable validity in the case of electricity markets because it does not take into account the size of the regions or the capacity of the transmission links between them. Despite these problems, for continuity and comparability with the empirical spatial econometric literature we estimate the model using a geographical weight matrix, without the row-normalization, as shown in Table 2-1. In our formulation of the geographical weight matrix, $W_{i j}=1$ if $i$ and $j$ have a common border of non-zero length. Clearly we would prefer a different formulation, one that is more attuned to the physical realities of the transmission system.

The "perfect" spatial weight matrix would contain a detailed engineering model of the entire system, and use all of the real-time engineering data available to the system operator, an informational burden that would be impracticable for virtually all researchers. Fortunately, the needs of the system operator far exceed the practical needs of the economic researchers and practitioners, who typically seek only to estimate parameters that express average relationships among variables over time. A reasonable compromise is the electrical contiguity weight matrix shown in Table 2-2 in which $\mathrm{W}_{i j}$ equals the transmission line capacity connecting region $i$ and $j$. Transmission line capacity is defined for purposes of Table 2-2 as the simple sum of the kilovolt ratings of all lines connecting nodes on two sides of the regional border, and was calculated by inspection of a system map provided by PJM. Various normalizations could be considered, such as division of each element in row $i$ by the row total, or perhaps by the maximum load or generation capacity in zone $i$, depending on the purposes and preferences of the researcher. For our own purpose (demonstrating the technique) we did not normalize the weights.

Anyone with even a cursory knowledge of the physics of electricity transmission systems knows that the system is dynamic and the patterns of constraints and interactions changes constantly. As transmission congestion becomes more severe and as transmission marginal costs grow, the electricity market becomes less and less integrated and the degree of spatial dependence falls. This means that during periods of high demand and/or periods of transmission congestion or 
failure, the spatial correlation has weaker effect on price formation than during periods of normal operation. A researcher should be uncomfortable, therefore, with the assumption of constant interactions embodied in the specification of a constant spatial interaction matrix $\rho \mathrm{W}$. To help address this concern, we stratified our sample of hourly price data according to the number of constraint contingencies occurring in the system in the hour in question, using data available online from PJM. As Table 2-3 shows, constraints are more numerous during peak demand hours. We expect the degree of spatial interaction to decline during periods with more constraint contingencies, a change that we accommodate by allowing the value of $\rho$ to change with the number of constraints. We expect $\rho$ to fall as the number of constraints rises.

\section{III.2 Panel Data}

Spatial panel data models are a recently developed but increasingly important tool in the econometric toolkit. Although they are methodologically challenging, spatial panel data models use more information. They therefore have a greater capacity for estimation of complex models than either the cross-section models that have predominated in the spatial literature or the time series models that have predominated in the empirical literature of electricity prices. The advantages of panel data include more accurate inference of model parameters due to more degrees of freedom and more sample variability, better predictive power, and greater capacity for uncovering dynamics. To our knowledge, spatial panel data methodology has not been applied previously to model electricity price dynamics. To demonstrate the use of these methods for electricity data, we estimate the parameters of our model using the spatial GMM panel data procedure described in Kapoor, Kelejian, and Prucha (2007). Many refinements to the techniques for various data sets and purposes are conceivable and desirable, but are beyond the scope of this article.

\section{Application}

\section{IV.1 Data}


The primary data set for this study consists of hourly real-time spot and day-ahead prices in the twelve zones (Allegheny Power, Atlantic City Electric, Baltimore Gas and Electric, Delmarva Power and Light, Jersey Central Power and Light, Metropolitan Edison, PECO Energy, PPL Electric Utilities, Pennsylvania Electric, Potomac Electric Power, Public Service Electric and Gas, and Rockland Electric) that existed in the PJM Interconnection from April 01, 2002 to May 31, 2006. Zone prices are weighted-averages of the LMPs at all nodes within the zone. For each of the 1522 days in the total sample, we have information on the prices (quoted in \$/MWh) for each of the 24 hours, downloaded from the PJM website.

Temperature data are hourly readings from 13 weather stations located in the PJM Interconnection area, weighted appropriately so as to characterize temperatures in the 12 zones. They are taken from the Global Summary of the Day (GSOD) database archived by the National Climatic Data Center (NCDC), and compiled and cleaned by researchers at ZedX, Inc, Atmospheric Sciences Division ${ }^{3}$.

Table 2-4 contains the summary statistics for the average hourly real-time and day-ahead prices (LMPs) for each of the twelve PJM zones. The importance of price spikes in electricity spot price distributions is confirmed by the high positive skewness, low median relative to mean prices, and high levels of excess kurtosis. All of these measures are lower for day-ahead forward prices, which are not sensitive to unexpected peaks in demand, failures of generation equipment, or realtime constraints in the transmission system. Minimum prices are negative; a consequence of the inability to freely dispose of electricity coupled with the costs of cycling baseload generators.

Besides LMPs by zone, PJM reports data on real-time transmission constraints, including the start hour, end hour, and duration of each contingency. Using this information we generated a data set containing hourly information on the number of constraint contingencies. These contingencies may be caused by lines reaching their thermal or voltage limits, or by other failures. Table 2-3 summarizes the distribution of number of congestion reports by time of day. The

\footnotetext{
${ }^{3}$ We would like to express our gratitude to Jerald Fletcher
} 
numbers in the table indicate the number of hours that the indicated number of transmission constraints was reported in PJM. Alternatively, the numbers may be thought of as the number of days (out of 1522 total) that the indicated number of transmission constraints was observed at the indicated time of day. Not surprisingly, the highest number of constraints is reported during the peak hours, especially noon through 10:00 PM, with a maximum at 3:00-6:00 PM. There were no transmission constraints reported in $29 \%$ of all hours during this period, and only one constraint in the system during an additional $27 \%$ of the hours. The fewest uncongested days are reported at noon, and the highest number of uncongested days was reported at 4:00 AM. Although the number of transmission contingencies is an inexact measure of system distress, it does provide information on the state of the system and the level of interconnectivity, and we use it to stratify our analysis.

Table 2-5 and Table 2-6 summarize key statistics of real-time and day-ahead hourly zonal prices for each number of constraint contingencies. The minimum, average, maximum, standard deviation, skewness, and kurtosis grow with the number of constraint contingencies. The coefficient of variation falls as the number of constraints increases, meaning that as the system becomes more constrained price volatility grows more slowly than the average price grows.

\section{IV.2 Electricity Prices Characteristics}

Electricity prices are also characterized by occasional abrupt and extreme changes or spikes over short periods of time. The spikes mostly occur during periods when consumption is high and the system is constrained. Skewness, variance, and kurtosis of electricity prices all increase during periods of system stress and high prices. The "spiky" nature of electricity prices is the result of the non-storability of electricity and limits to production and transmission capacity. These distributional peculiarities make it difficult to justify the use of maximum likelihood techniques that assume stable, and usually normal, error distributions.

One approach to making estimation more tractable is to pre-filter the price data using time series tools to account for the volatility clustering, skewness, and excess kurtosis that characterize electricity prices. Filtering could in principle be done simultaneously with the spatial modeling by 
specifying a sufficiently complex likelihood function, but as a practical matter it is preferable to perform sequential filtering using available software, if such a procedure can be done in a way that produces consistent estimators. Under the SAR error specification, pre-filtering is permissible. Because $x$ is exogenous, estimation of equation (1) using OLS, or GARCH or other methods that ignore spatial effects will provide an unbiased and consistent, but inefficient estimator of $\beta$, despite the SAR error structure. The spatial model can then be estimated by specifying a likelihood function that takes the estimated time series characteristics into account, if an argument can be made that the remaining errors conform to a normal or other identifiable distribution. As Anselin (1988) points out, the spatial parameter $\rho$ itself can not be estimated consistently using feasible GLS procedures, but there is nothing to preclude pre-filtering of the errors in (1) prior to estimating the spatial effects.

The use of time series filtering techniques to "whiten" electricity price series has met with limited success, however. There is a large recent literature on time series models of electricity prices (for a survey see Knittel and Roberts, 2005) that mostly focuses on market structure and market power, cost and engineering factors, impact of deregulation on markets, and modeling electricity price dynamics. These papers include Arciniegas et al. (2003), Bessembinder and Lemmon (2002), Bhanot (2000), Huisman and Mahieu (2003), Deng (1999), DeVany and Walls (1999a, 1999b), Feng et al. (2007), Higgs and Worthington (2003), Li and Flynn (2004), Longstaff and Wang (2004), Lucia and Schwartz (2002), Parket al. (2006), Shawky et al. (2003), Weron et al. (2004), Weron and Przybylowicz (2000), Weron (2006), and Worthington and Higgs (2004).

No time-series filtering technique has been developed that can take electricity prices and reliably generate residuals whose pattern resembles a normal distribution. This fact complicates or even precludes the valid use of traditional spatial maximum likelihood techniques when working with electricity prices. We therefore take a different approach and use a variant of the spatial generalized method of moments (GMM) estimator first derived by Kelejian and Prucha (1998, 1999). The distributional assumptions required for valid application of a GMM estimator are much less restrictive than those required for maximum likelihood. 
Although pre-filtering could be used to improve the properties of the GMM estimator, as described below we control for most of the peculiarities of electricity spot prices by using day-ahead forward market prices as a predetermined $\mathrm{x}$ variable, and use hourly temperature data to control for most of the rest of the variation. Although day-ahead prices are biased estimates of spot prices, as described by Bessembinder and Lemmon (2002) and Douglas and Popova (2008), it is reasonable to use them as exogenous variables, since their levels are driven by the same factors as the realtime prices and they are known to the short-term forecaster, but are predetermined with respect to real-time prices.

Seasonality, or predictable cyclical fluctuation over time, is one of the most pronounced features of electricity prices, and is driven primarily by the exogenous and regular patterns of demand shifts. There are three levels of seasonality in electricity prices depicted on Figure 2-1: diurnal, weekly and annual. Diurnal seasonality (which causes daily off-peak and on-peak price patterns) can be explained by the large change in consumption between daytime and nighttime hours as people go through their natural daily cycle of sleep and activity. Weekly seasonality arises due to differences in commercial and industrial activity between work days and weekends, and extends in a predictable manner to holidays as well. Annual seasonality is driven largely by cycles of space heating and cooling due to weather conditions such as temperature, humidity, and wind speed. Electricity prices are highest during summer when air conditioning is needed, and during winter when electricity is used for space heating.

There are a variety of ways of dealing with seasonality in time series in general, and electricity price data in particular. Some researchers use dummy variables; others control for the daily cycle by estimating each hour as a separate time series. We again assume that forward price formation includes a best estimate of the cyclical components, and we therefore use the day-ahead forward price as a predetermined variable. This assumption is supported by the close relationship between the seasonal patterns of forward (DA) and spot (RT) prices in Figure 2-1. Further control for seasonality is achieved through the use of the temperature variables. 


\section{IV.3 Empirical Model and Results}

To provide a concrete demonstration of the application of a spatial econometric model to electricity prices, we estimated a simple model of electricity prices. There are $N=12$ cross-sectional units, or zones, observed over $T$ time periods. Our empirical model is

$$
\text { Spot }_{t i}=\beta_{1}+\beta_{2} F_{t i}+\beta_{3} T e m p_{t i}+\beta_{4} T e m p^{2} t i+\varepsilon_{t i}
$$

where $F_{t i}$ is the day-ahead forward price for the hour, date, and region corresponding to Spot $_{t i}$, and Temp $t i$ is the temperature in degrees Fahrenheit at that time and place. The quadratic form allows for the marginal effect of temperature to change, as is generally observed. In setting up and estimating the spatial error model we followed the specification of Kapoor et al. (2007), which expresses the NT by 1 disturbance vector $\varepsilon$ using kronecker product notation:

$$
\varepsilon_{N}=\left[I_{T} \otimes\left(I_{N}-\rho W\right)^{-1}\right] v_{N}
$$

where $\mathrm{I}_{\mathrm{T}}$ is the $T$ by $T$ identity matrix, $\mathrm{I}_{\mathrm{N}}$ is the $N$ by $N$ identity matrix, the $N T$ by 1 column vector $\mathrm{u}_{\mathrm{N}}$ contains the spatially correlated errors, and $\mathrm{W}$ is the $N$ by $N$ spatial weight matrix. The data are stratified by the number of constraint contingencies, so $T$ varies from 83 to 10,672 .

The results of estimating the SAR in errors model are presented in Table 2-7 and Table 2-8. First, unsurprisingly but reassuringly, the results do indicate that real-time electricity prices exhibit spatial correlation. The estimates of $\rho$ vary from 0.92 to 0.71 for the geographical contiguity weight matrix, and from 0.85 to 0.61 for the electrical contiguity weight matrix, indicating strong spatial correlation that decreases as more transmission lines become congested. Asymptotic $t$ statistics indicate that $\rho$ is significantly less than one, and always greater than zero, at any reasonable significance level for all levels of constraints in the system. Spatial dependence of prices in the PJM interconnection market appears to be econometrically important, and it can not be ignored in accurate price modeling.

As expected, the spatial correlation generally weakens as the system becomes more congested. Figure 2-2 and Figure 2-3 indicate that the estimate of spatial coefficient $\rho$ is high for non-constrained hours and drops at a decreasing rate as the number of constraints increases. This 
can be explained by the fact that when more transmission lines are congested, it becomes more difficult or impossible to deliver power across the zonal boundaries. One consequence of the physics of transmission line congestion is that even though the lines that connect two transmission zones may be not constrained, severe congestion in other places in the system (including lines strictly interior to one of the zones) may prevent free power travel across boundaries, decreasing the degree to which zones are spatially correlated.

The value of estimated $\rho$ decreases monotonically up to seven contingencies and then levels off for higher levels of contingencies. The decline in $\rho$ for the case of eleven constraint contingencies may be due to the relatively few observations for this category. High numbers of constraints indicate a high level of system distress, and when the system is highly distressed prices tend to rise quickly in most zones at once, either because of extensive islanding and pervasive outof-merit dispatch, or because of increased opportunities to exercise market power. Thus, the slight upturn in $\rho$ for twelve or more constraints may reflect sampling error or extreme outliers.

For comparison to the spatial model results, we report the results of a random-effects panel estimation model estimated in the usual way, without spatial modeling, in Table 2-9. (A Hausman test supported the use of the random effects model rather than the less efficient fixed-effects.) Compare the patterns of coefficients on the forward price variable in tables Table 2-7, Table 2-8 , and Table 2-9. When the system has zero constraints there is relatively little variation in the forward price (cf. tables Table 2-4 and Table 2-5 and Table 2-6), and the spatial estimator attributes price formation to spatial correlation, as indicated by the relatively high value of $\hat{\rho}$ and the relatively low forward price coefficient for zero constraints. As the system becomes slightly more constrained and more variation occurs in forward and spot prices, the forward price coefficient in the spatial model rises to one, as would be expected if the forward price is reasonably accurate. The spatial model estimate stays closer to one than the non-spatial estimate until the number of constraints rises to nine. With more constraints on the system, spot prices become harder to predict, the spatial patterns of interaction begin to break down, and the spatial and non-spatial models' estimates appear to converge. 


\section{Summary and Conclusion}

There is no doubt that information about the characteristics of the transmission grid is relevant to understanding and predicting electricity prices at different points on the grid. The large and growing empirical literature on electricity prices, however, has largely ignored this information. In this article we have argued that spatial econometric methods provide a promising approach to incorporating this information into empirical models. We have discussed the formal characteristics of spatial econometric models in the context of the econometric problems endemic to electricity price data, and have provided an illustration of the application of a spatial model to PJM price data. Our empirical model is quite simple, yet it incorporates a crude model of the relevant transmission system, and provides some information about the effect of system constraints on the extent of electricity market integration.

Our empirical results confirm both the existence of spatial patterns in electricity prices and the ability of spatial econometric methods to detect those patterns, and they show that incorporating a spatial component into an econometric model of electricity prices can significantly alter parameter estimates. We discuss the flexibility of spatial econometric methods for modeling the structure that produces those patterns, and discuss how the time series filtering methods that have heretofore dominated the empirical electricity price literature might be incorporated into a spatial econometric model. We also illustrate the use of panel data methods that are at the cutting edge of spatial econometrics.

Future work in this area will be required to adapt spatial methods to the many and disparate purposes of the community of scholars and practitioners who are interested in electricity prices. Basic work in econometric theory needs to be done to adapt current spatial econometric panel data models so that they can take better advantage of the long time series component of electricity price data. We look forward to future advances in our ability to use the information inherent in spatial patterns in electricity prices. 
Table 2-1: Geographical Contiguity Spatial Weight Matrix

\begin{tabular}{|l|l|l|l|l|l|l|l|l|l|l|l|}
\hline 0 & 1 & 0 & 1 & 0 & 0 & 1 & 1 & 0 & 0 & 1 & 0 \\
\hline 1 & 0 & 0 & 1 & 0 & 0 & 0 & 1 & 0 & 0 & 0 & 0 \\
\hline 0 & 0 & 0 & 1 & 1 & 1 & 0 & 1 & 0 & 0 & 1 & 0 \\
\hline 1 & 1 & 1 & 0 & 0 & 1 & 0 & 1 & 0 & 1 & 1 & 0 \\
\hline 0 & 0 & 1 & 0 & 0 & 1 & 0 & 0 & 1 & 1 & 0 & 0 \\
\hline 0 & 0 & 1 & 1 & 1 & 0 & 0 & 1 & 1 & 1 & 1 & 0 \\
\hline 1 & 0 & 0 & 0 & 0 & 0 & 0 & 0 & 0 & 0 & 1 & 0 \\
\hline 1 & 1 & 1 & 1 & 0 & 1 & 0 & 0 & 1 & 1 & 1 & 0 \\
\hline 0 & 0 & 0 & 0 & 1 & 1 & 0 & 1 & 0 & 1 & 0 & 1 \\
\hline 0 & 0 & 0 & 1 & 1 & 1 & 0 & 1 & 1 & 0 & 0 & 1 \\
\hline 1 & 0 & 1 & 1 & 0 & 1 & 1 & 1 & 0 & 0 & 0 & 0 \\
\hline 0 & 0 & 0 & 0 & 0 & 0 & 0 & 0 & 1 & 1 & 0 & 0 \\
\hline
\end{tabular}

Zones corresponding to columns and rows are: Allegheny Power, Atlantic City Electric, Baltimore Gas and Electric, Delmarva Power and Light, Jersey Central Power and Light, Metropolitan Edison, PECO Energy, PPL Electric Utilities, Pennsylvania Electric, Potomac Electric Power, Public Service Electric and Gas, Rockland Electric

Table 2-2: Electrical Contiguity Spatial Weight Matrix

\begin{tabular}{|r|r|r|r|r|r|r|r|r|r|r|r|r|}
\hline 0 & 2472 & 0 & 0 & 0 & 0 & 1920 & 138 & 0 & 0 & 0 & 0 \\
\hline 2472 & 0 & 0 & 1460 & 0 & 0 & 0 & 615 & 0 & 0 & 0 & 0 \\
\hline 0 & 0 & 0 & 0 & 500 & 1170 & 0 & 0 & 250 & 0 & 0 & 0 \\
\hline 0 & 1460 & 0 & 0 & 0 & 0 & 0 & 5048 & 1000 & 960 & 230 & 0 \\
\hline 0 & 0 & 500 & 0 & 0 & 230 & 0 & 0 & 1420 & 0 & 0 & 0 \\
\hline 0 & 0 & 1170 & 0 & 230 & 0 & 250 & 1440 & 966 & 0 & 345 & 0 \\
\hline 1920 & 0 & 0 & 0 & 0 & 250 & 0 & 0 & 0 & 0 & 2420 & 0 \\
\hline 138 & 615 & 0 & 5048 & 0 & 1440 & 0 & 0 & 250 & 1150 & 0 & 0 \\
\hline 0 & 0 & 250 & 1000 & 1420 & 966 & 0 & 250 & 0 & 3726 & 0 & 1130 \\
\hline 0 & 0 & 0 & 960 & 0 & 0 & 0 & 1150 & 3726 & 0 & 0 & 1190 \\
\hline 0 & 0 & 0 & 230 & 0 & 345 & 2420 & 0 & 0 & 0 & 0 & 0 \\
\hline 0 & 0 & 0 & 0 & 0 & 0 & 0 & 0 & 1130 & 1190 & 0 & 0 \\
\hline
\end{tabular}

Zones corresponding to columns and rows are: Allegheny Power, Atlantic City Electric, Baltimore Gas and Electric, Delmarva Power and Light, Jersey Central Power and Light, Metropolitan Edison, PECO Energy, PPL Electric Utilities, Pennsylvania Electric, Potomac Electric Power, Public Service Electric and Gas, Rockland Electric 
Table 2-3: Number of Constraint Contingencies by Time of a Day

\begin{tabular}{|c|c|c|c|c|c|c|c|c|c|c|c|c|c|}
\hline Hour & None & One & Two & Three & Four & Five & Six & Seven & Eight & Nine & Ten & Eleven & Twelve+ \\
\hline $\mathbf{1}$ & 577 & 481 & 239 & 133 & 59 & 19 & 10 & 1 & 3 & 0 & 0 & 0 & 0 \\
\hline $\mathbf{2}$ & 602 & 501 & 238 & 109 & 43 & 17 & 7 & 3 & 2 & 0 & 0 & 0 & 0 \\
\hline $\mathbf{3}$ & 637 & 493 & 225 & 95 & 43 & 24 & 2 & 2 & 1 & 0 & 0 & 0 & 0 \\
\hline $\mathbf{4}$ & 657 & 489 & 218 & 92 & 37 & 20 & 7 & 1 & 1 & 0 & 0 & 0 & 0 \\
\hline $\mathbf{5}$ & 604 & 503 & 223 & 104 & 59 & 19 & 7 & 0 & 3 & 0 & 0 & 0 & 0 \\
\hline $\mathbf{6}$ & 544 & 488 & 229 & 133 & 62 & 38 & 21 & 4 & 2 & 1 & 0 & 0 & 0 \\
\hline $\mathbf{7}$ & 514 & 440 & 262 & 127 & 99 & 41 & 25 & 8 & 6 & 0 & 0 & 0 & 0 \\
\hline $\mathbf{8}$ & 506 & 424 & 270 & 158 & 88 & 41 & 20 & 7 & 6 & 2 & 0 & 0 & 0 \\
\hline $\mathbf{9}$ & 449 & 442 & 288 & 168 & 89 & 59 & 16 & 6 & 2 & 1 & 2 & 0 & 0 \\
\hline $\mathbf{1 0}$ & 371 & 415 & 298 & 216 & 113 & 52 & 34 & 12 & 5 & 2 & 3 & 1 & 0 \\
\hline $\mathbf{1 1}$ & 327 & 387 & 309 & 215 & 133 & 68 & 39 & 19 & 9 & 7 & 4 & 5 & 0 \\
\hline $\mathbf{1 2}$ & 301 & 389 & 293 & 207 & 139 & 77 & 38 & 35 & 14 & 13 & 8 & 3 & 5 \\
\hline $\mathbf{1 3}$ & 304 & 379 & 289 & 208 & 132 & 75 & 49 & 25 & 14 & 23 & 14 & 4 & 6 \\
\hline $\mathbf{1 4}$ & 313 & 350 & 293 & 203 & 126 & 77 & 56 & 30 & 21 & 16 & 14 & 10 & 13 \\
\hline $\mathbf{1 5}$ & 334 & 364 & 261 & 186 & 135 & 79 & 48 & 29 & 25 & 20 & 14 & 8 & 19 \\
\hline $\mathbf{1 6}$ & 330 & 382 & 256 & 187 & 127 & 70 & 48 & 35 & 20 & 22 & 11 & 13 & 21 \\
\hline $\mathbf{1 7}$ & 304 & 346 & 268 & 210 & 129 & 88 & 44 & 40 & 32 & 19 & 13 & 12 & 17 \\
\hline $\mathbf{1 8}$ & 305 & 297 & 278 & 195 & 158 & 113 & 44 & 47 & 25 & 15 & 15 & 10 & 20 \\
\hline $\mathbf{1 9}$ & 333 & 343 & 272 & 196 & 140 & 90 & 42 & 32 & 19 & 16 & 15 & 10 & 14 \\
\hline $\mathbf{2 0}$ & 383 & 392 & 261 & 192 & 117 & 72 & 29 & 34 & 11 & 15 & 6 & 3 & 7 \\
\hline $\mathbf{2 1}$ & 400 & 415 & 280 & 185 & 102 & 66 & 32 & 18 & 7 & 7 & 4 & 1 & 5 \\
\hline $\mathbf{2 2}$ & 464 & 418 & 293 & 150 & 103 & 44 & 22 & 12 & 7 & 4 & 1 & 3 & 1 \\
\hline $\mathbf{2 3}$ & 560 & 416 & 272 & 145 & 71 & 38 & 12 & 3 & 2 & 2 & 1 & 0 & 0 \\
\hline $\mathbf{2 4}$ & 553 & 468 & 263 & 131 & 67 & 24 & 10 & 6 & 0 & 0 & 0 & 0 & 0 \\
\hline Total & $\mathbf{1 0 6 7 2}$ & $\mathbf{1 0 0 2 2}$ & $\mathbf{6 3 7 8}$ & $\mathbf{3 9 4 5}$ & $\mathbf{2 3 7 1}$ & $\mathbf{1 3 1 1}$ & $\mathbf{6 6 2}$ & $\mathbf{4 0 9}$ & $\mathbf{2 3 7}$ & $\mathbf{1 8 5}$ & $\mathbf{1 2 5}$ & $\mathbf{8 3}$ & $\mathbf{1 2 8}$ \\
\hline
\end{tabular}


Table 2-4: Summary Statistics for Hourly Electricity Prices

\begin{tabular}{|c|c|c|c|c|c|c|c|c|c|c|c|c|}
\hline \multicolumn{13}{|c|}{ Real-Time Prices, 01 Apr 2002 -- 31 May 2006} \\
\hline & Zone1 & Zone2 & Zone3 & Zone4 & Zone5 & Zone6 & Zone7 & Zone8 & Zone9 & Zone10 & Zone11 & Zone12 \\
\hline Min & -90.92 & -19.73 & -20.75 & -49.36 & -24.84 & -21.32 & -14.41 & -19.31 & -25.71 & -5.21 & -18.72 & -180.84 \\
\hline Mean & 48.69 & 47.69 & 47.35 & 47.28 & 45.96 & 46.61 & 43.15 & 48.28 & 44.94 & 49.28 & 42.43 & 48.13 \\
\hline Median & 40.06 & 38.20 & 39.11 & 38.58 & 37.93 & 38.71 & 36.73 & 38.25 & 37.48 & 40.32 & 35.51 & 40.10 \\
\hline Max & 764.01 & 1162.70 & 873.76 & 701.71 & 760.61 & 762.06 & 372.58 & 1293.61 & 713.12 & 702.19 & 499.03 & 639.40 \\
\hline $\begin{array}{l}\text { Standard } \\
\text { Deviation }\end{array}$ & 34.72 & 36.66 & 33.83 & 34.72 & 32.74 & 33.03 & 27.40 & 38.24 & 31.64 & 35.00 & 28.43 & 33.22 \\
\hline $\begin{array}{l}\text { Coefficient of } \\
\text { Skewness }\end{array}$ & 2.34 & 3.37 & 2.70 & 2.27 & 2.49 & 2.59 & 1.93 & 3.71 & 2.45 & 2.31 & 2.19 & 2.17 \\
\hline Kurtosis & 18.32 & 40.76 & 25.50 & 15.73 & 18.86 & 21.52 & 0.07 & 50.25 & 18.07 & 16.33 & 2.66 & 14.32 \\
\hline $\begin{array}{c}\text { Coefficient of } \\
\text { Variation }\end{array}$ & 0.71 & 0.77 & 0.71 & 0.73 & 0.71 & 0.71 & 0.64 & 0.79 & 0.70 & 0.71 & 0.67 & 0.69 \\
\hline \multicolumn{13}{|c|}{ Day-Ahead Prices, 01 Apr 2002 -- 31 May 2006} \\
\hline & Zone1 & Zone2 & Zone3 & Zone4 & Zone5 & Zone6 & Zone7 & Zone8 & Zone9 & Zone10 & Zone11 & Zone12 \\
\hline Min & -0.21 & 0.00 & -0.14 & -4.48 & -1.34 & -0.26 & 0.00 & 0.00 & -1.31 & 0.00 & 0.00 & 0.00 \\
\hline Mean & 48.70 & 46.63 & 47.64 & 47.23 & 46.00 & 47.08 & 43.40 & 47.18 & 45.23 & 48.68 & 42.31 & 47.46 \\
\hline Median & 43.65 & 40.93 & 42.96 & 42.26 & 40.74 & 42.45 & 39.43 & 41.09 & 40.36 & 44.00 & 37.88 & 43.33 \\
\hline Max & 347.16 & 256.54 & 326.08 & 258.21 & 257.30 & 458.87 & 453.88 & 279.16 & 250.00 & 419.92 & 225.60 & 231.05 \\
\hline $\begin{array}{l}\text { Standard } \\
\text { Deviation }\end{array}$ & 28.81 & 28.12 & 27.75 & 27.52 & 27.08 & 27.63 & 22.95 & 29.07 & 26.46 & 27.83 & 23.77 & 26.20 \\
\hline $\begin{array}{c}\text { Coefficient of } \\
\text { Skewness }\end{array}$ & 1.61 & 1.70 & 1.67 & 1.46 & 1.69 & 1.79 & 1.93 & 1.81 & 1.68 & 1.55 & 1.76 & 1.46 \\
\hline Kurtosis & 7.69 & 7.59 & 8.03 & 6.53 & 7.68 & 9.94 & 15.60 & 8.26 & 7.64 & 7.57 & 8.24 & 6.65 \\
\hline $\begin{array}{c}\text { Coefficient of } \\
\text { Variation }\end{array}$ & 0.59 & 0.60 & 0.58 & 0.58 & 0.59 & 0.59 & 0.53 & 0.62 & 0.58 & 0.57 & 0.56 & 0.55 \\
\hline
\end{tabular}

Note: Coefficient of Variation is the standard deviation divided by the mean

Zones 1-12 consist of: Allegheny Power, Atlantic City Electric, Baltimore Gas and Electric, Delmarva Power and Light, Jersey Central

Power and Light, Metropolitan Edison, PECO Energy, PPL Electric Utilities, Pennsylvania Electric, Potomac Electric Power, Public Service Electric and Gas, and Rockland Electric 
Table 2-5: Summary Statistics by Number of Constraints for Spot and Day-Ahead Prices Averaged Over Zones

\begin{tabular}{|c|c|c|c|c|c|c|}
\hline \multicolumn{7}{|c|}{ Real-Time Prices, 01 Apr 2002 - 31 May 2006 } \\
\hline Number of Constraints & None & One & Two & Three & Four & Five \\
\hline Min & -4.35 & -12.21 & -40.93 & -16.54 & -3.18 & -6.43 \\
\hline Mean & 33.05 & 39.23 & 48.08 & 55.87 & 65.18 & 74.67 \\
\hline Median & 25.99 & 33.82 & 41.63 & 48.04 & 55.57 & 65.48 \\
\hline Max & 171.94 & 226.76 & 288.77 & 265.73 & 437.53 & 337.49 \\
\hline Standard Deviation & 22.51 & 23.62 & 27.93 & 30.88 & 37.13 & 40.08 \\
\hline Coefficient of Skewness & 1.54 & 1.69 & 1.92 & 1.63 & 1.99 & 1.62 \\
\hline Kurtosis & 6.10 & 7.71 & 9.72 & 7.12 & 11.57 & 7.33 \\
\hline Coefficient of Variation & 0.68 & 0.60 & 0.58 & 0.55 & 0.57 & 0.54 \\
\hline \multicolumn{6}{|c|}{ Day-Ahead Prices, 01 Apr 2002 --31 May 2006 } & \\
\hline Number of Constraints & None & One & Two & Three & Four & Five \\
\hline Min & -0.65 & 0.00 & 5.24 & 10.83 & 13.66 & 15.06 \\
\hline Mean & 35.11 & 40.18 & 47.47 & 53.84 & 60.92 & 69.30 \\
\hline Median & 30.82 & 36.87 & 44.01 & 49.00 & 55.40 & 62.34 \\
\hline Max & 157.35 & 167.25 & 215.94 & 206.06 & 217.09 & 230.15 \\
\hline Standard Deviation & 19.66 & 20.24 & 22.22 & 24.32 & 26.47 & 30.43 \\
\hline Coefficient of Skewness & 1.20 & 1.27 & 1.38 & 1.40 & 1.34 & 1.30 \\
\hline Kurtosis & 5.23 & 5.75 & 6.40 & 5.90 & 5.69 & 5.69 \\
\hline Coefficient of Variation & 0.56 & 0.50 & 0.47 & 0.45 & 0.43 & 0.44 \\
\hline
\end{tabular}

Note: Coefficient of Variation is the standard deviation divided by the mean. Electricity Prices are averaged over twelve zones 
Table 2-6: Summary Statistics by Number of Constraints for Spot and Day-Ahead Prices Averaged Over Zones

\begin{tabular}{|c|c|c|c|c|c|c|c|}
\hline \multicolumn{8}{|c|}{ Real-Time Prices, 01 Apr 2002 -- 31 May 2006} \\
\hline Number of Constraints & Six & Seven & Eight & Nine & Ten & Eleven & Twelve+ \\
\hline Min & 9.85 & 14.67 & 26.60 & 26.01 & 34.45 & 27.15 & 23.63 \\
\hline Mean & 81.92 & 90.03 & 106.43 & 103.04 & 113.84 & 138.38 & 137.91 \\
\hline Median & 73.00 & 78.33 & 90.67 & 91.79 & 102.31 & 111.65 & 132.20 \\
\hline Max & 437.39 & 290.70 & 463.51 & 259.51 & 364.44 & 770.40 & 334.13 \\
\hline Standard Deviation & 43.53 & 45.22 & 58.33 & 46.65 & 56.01 & 108.18 & 60.01 \\
\hline Coefficient of Skewness & 2.08 & 1.26 & 1.97 & 0.98 & 1.49 & 3.51 & 0.45 \\
\hline Kurtosis & 12.33 & 4.84 & 9.65 & 3.61 & 6.43 & 19.40 & 3.12 \\
\hline Coefficient of Variation & 0.531 & 0.50 & 0.55 & 0.45 & 0.49 & 0.77 & 0.44 \\
\hline \multicolumn{8}{|c|}{ Day-Ahead Prices, 01 Apr 2002 -- 31 May 2006} \\
\hline Number of Constraints & Six & Seven & Eight & Nine & Ten & Eleven & Twelve+ \\
\hline Min & 15.23 & 21.40 & 33.02 & 41.39 & 47.70 & 59.12 & 62.15 \\
\hline Mean & 76.97 & 85.39 & 97.46 & 105.94 & 110.96 & 116.61 & 134.49 \\
\hline Median & 70.59 & 75.76 & 92.33 & 102.39 & 106.50 & 107.31 & 127.57 \\
\hline Max & 237.33 & 252.41 & 222.29 & 243.56 & 224.11 & 251.03 & 296.68 \\
\hline Standard Deviation & 31.49 & 35.55 & 35.49 & 35.68 & 36.24 & 39.57 & 46.06 \\
\hline Coefficient of Skewness & 1.06 & 1.27 & 0.73 & 0.80 & 0.82 & 1.34 & 1.12 \\
\hline Kurtosis & 4.68 & 4.95 & 3.52 & 3.94 & 4.35 & 6.65 & 5.40 \\
\hline Coefficient of Variation & 0.41 & 0.42 & 0.36 & 0.34 & 0.33 & 0.34 & 0.34 \\
\hline
\end{tabular}

Note: Coefficient of Variation is the standard deviation divided by the mean. Electricity Prices are averaged over twelve zones 
Table 2-7: Spatial Panel Econometric Estimation Results, Geographical Contiguity Weight Matrix

Random Effects Panel Estimation

Dependent Variable: Spot Price

\begin{tabular}{|c|c|c|c|c|c|c|}
\hline \# Constraints & $\rho$ & $\mathbf{F}$ & $T$ & $\mathrm{~T}^{2}$ & Adj-R ${ }^{2}$ & \# Obs \\
\hline None & $\begin{array}{c}\mathbf{0 . 9 1 8} \\
33.655\end{array}$ & $\begin{array}{c}\mathbf{0 . 2 3 8} \\
130.093\end{array}$ & $\begin{array}{l}-0.035 \\
-7.428\end{array}$ & $\begin{array}{c}\mathbf{0 . 0 0 0 1} \\
1.931\end{array}$ & $28.97 \%$ & 10672 \\
\hline One & $\begin{array}{c}\mathbf{0 . 8 5 6} \\
24.433\end{array}$ & $\begin{array}{c}\mathbf{0 . 6 5 9} \\
187.948\end{array}$ & $\begin{array}{c}-0.272 \\
-20.546\end{array}$ & $\begin{array}{c}\mathbf{0 . 0 0 3} \\
20.889\end{array}$ & $50.01 \%$ & 10022 \\
\hline Two & $\begin{array}{c}\mathbf{0 . 8 3 6} \\
21.216\end{array}$ & $\begin{array}{c}0.94 \\
241.631\end{array}$ & $\begin{array}{c}-0.256 \\
-11.605\end{array}$ & $\begin{array}{c}\mathbf{0 . 0 0 3} \\
13.484\end{array}$ & $47.64 \%$ & 6378 \\
\hline Three & $\begin{array}{l}\mathbf{0 . 8 1 9} \\
16.44\end{array}$ & $\begin{array}{c}1.086 \\
243.958\end{array}$ & $\begin{array}{l}-0.134 \\
-4.285\end{array}$ & $\begin{array}{l}\mathbf{0 . 0 0 2} \\
7.323\end{array}$ & $43.70 \%$ & 3945 \\
\hline Four & $\begin{array}{c}\mathbf{0 . 8 4} \\
13.293\end{array}$ & $\begin{array}{c}1.216 \\
228.075\end{array}$ & $\begin{array}{l}-0.098 \\
-2.274\end{array}$ & $\begin{array}{c}\mathbf{0 . 0 0 2} \\
4.14\end{array}$ & $39.39 \%$ & 2371 \\
\hline Five & $\begin{array}{l}\mathbf{0 . 8 0 2} \\
8.527\end{array}$ & $\begin{array}{c}1.209 \\
143.667\end{array}$ & $\begin{array}{l}-0.196 \\
-2.913\end{array}$ & $\begin{array}{l}\mathbf{0 . 0 0 3} \\
5.105\end{array}$ & $38.52 \%$ & 1311 \\
\hline Six & $\begin{array}{l}\mathbf{0 . 8 2 1} \\
6.811\end{array}$ & $\begin{array}{c}1.2 \\
98.044\end{array}$ & $\begin{array}{l}-0.497 \\
-4.389\end{array}$ & $\begin{array}{l}\mathbf{0 . 0 0 7} \\
8.263\end{array}$ & $32.62 \%$ & 662 \\
\hline Seven & $\begin{array}{l}\mathbf{0 . 7 9 8} \\
5.216\end{array}$ & $\begin{array}{l}1.122 \\
65.96\end{array}$ & $\begin{array}{c}-1.22 \\
-5.753\end{array}$ & $\begin{array}{l}\mathbf{0 . 0 1 4} \\
9.003\end{array}$ & $42.44 \%$ & 409 \\
\hline Eight & $\begin{array}{l}\mathbf{0 . 8 4 4} \\
6.950\end{array}$ & $\begin{array}{c}0.949 \\
29.302\end{array}$ & $\begin{array}{l}-1.265 \\
-4.389\end{array}$ & $\begin{array}{l}\mathbf{0 . 0 1 5} \\
7.122\end{array}$ & $35.46 \%$ & 237 \\
\hline Nine & $\begin{array}{c}\mathbf{0 . 8 3} \\
34.113\end{array}$ & $\begin{array}{l}1.077 \\
43.89\end{array}$ & $\begin{array}{c}-1.203 \\
-1.97\end{array}$ & $\begin{array}{l}\mathbf{0 . 0 1 5} \\
3.973\end{array}$ & $30.02 \%$ & 185 \\
\hline Ten & $\begin{array}{l}0.824 \\
4.931\end{array}$ & $\begin{array}{c}\mathbf{0 . 7 1 8} \\
20.042\end{array}$ & $\begin{array}{c}-2.101 \\
-1.08\end{array}$ & $\begin{array}{l}\mathbf{0 . 0 2 1} \\
1.807\end{array}$ & $29.13 \%$ & 125 \\
\hline Eleven & $\begin{array}{l}0.705 \\
9.614\end{array}$ & $\begin{array}{l}1.407 \\
26.91\end{array}$ & $\begin{array}{l}-0.309 \\
-0.104\end{array}$ & $\begin{array}{l}0.02 \\
1.095\end{array}$ & $13.27 \%$ & 83 \\
\hline Twelv & $\begin{array}{c}\mathbf{0 . 8 3 1} \\
55.125\end{array}$ & $\begin{array}{c}\mathbf{0 . 8 2 1} \\
25.042\end{array}$ & $\begin{array}{l}-\mathbf{0 . 5 3 1} \\
-0.186\end{array}$ & $\begin{array}{l}\mathbf{0 . 0 2 2} \\
1.316 \\
\end{array}$ & $12.81 \%$ & 128 \\
\hline
\end{tabular}

Note: $t$ statistics are in italic 
Table 2-8: Spatial Panel Econometric Estimation Results, Electrical Contiguity Weight Matrix

Random Effects Panel Estimation

Dependent Variable: Spot Price

\begin{tabular}{|c|c|c|c|c|c|c|}
\hline \# Constraints & $\rho$ & $F$ & $T$ & $\mathrm{~T}^{2}$ & Adj- $R^{2}$ & \# Obs \\
\hline None & $\begin{array}{c}\mathbf{0 . 8 5 0} \\
40.543\end{array}$ & $\begin{array}{c}\mathbf{0 . 3 0 2} \\
173.660\end{array}$ & $\begin{array}{c}-0.093 \\
-17.219\end{array}$ & $\begin{array}{l}\mathbf{0 . 0 0 0 8} \\
13.388\end{array}$ & $35.22 \%$ & 10672 \\
\hline One & $\begin{array}{c}\mathbf{0 . 7 4 1} \\
36.300\end{array}$ & $\begin{array}{c}\mathbf{0 . 7 0 5} \\
246.294 \\
\end{array}$ & $\begin{array}{c}-0.367 \\
-28.017 \\
\end{array}$ & $\begin{array}{c}\mathbf{0 . 0 0 4} \\
29.840 \\
\end{array}$ & $51.16 \%$ & 10022 \\
\hline Two & $\begin{array}{c}\mathbf{0 . 6 8 9} \\
29.410\end{array}$ & $\begin{array}{c}\mathbf{0 . 9 0 7} \\
299.800\end{array}$ & $\begin{array}{c}-0.313 \\
-13.790\end{array}$ & $\begin{array}{c}\mathbf{0 . 0 0 4} \\
17.978\end{array}$ & $47.34 \%$ & 6378 \\
\hline Three & $\begin{array}{c}\mathbf{0 . 6 6 6} \\
22.961 \\
\end{array}$ & $\begin{array}{c}1.021 \\
291.939 \\
\end{array}$ & $\begin{array}{l}-0.024 \\
-0.772 \\
\end{array}$ & $\begin{array}{l}\mathbf{0 . 0 0 2} \\
6.910 \\
\end{array}$ & $43.50 \%$ & 3945 \\
\hline Four & $\begin{array}{c}\mathbf{0 . 6 9 7} \\
18.247\end{array}$ & $\begin{array}{c}1.089 \\
229.320\end{array}$ & $\begin{array}{l}\mathbf{0 . 0 9 6} \\
2.071 \\
\end{array}$ & $\begin{array}{l}0.002 \\
3.451\end{array}$ & $38.84 \%$ & 2371 \\
\hline Five & $\begin{array}{c}\mathbf{0 . 6 5 2} \\
12.339 \\
\end{array}$ & $\begin{array}{c}1.213 \\
166.631 \\
\end{array}$ & $\begin{array}{l}-0.167 \\
-2.377 \\
\end{array}$ & $\begin{array}{l}\mathbf{0 . 0 0 4} \\
6.000 \\
\end{array}$ & $35.90 \%$ & 1311 \\
\hline Six & $\begin{array}{l}\mathbf{0 . 6 8 9} \\
9.331 \\
\end{array}$ & $\begin{array}{c}1.037 \\
82.744 \\
\end{array}$ & $\begin{array}{l}-0.484 \\
-4.209 \\
\end{array}$ & $\begin{array}{l}\mathbf{0 . 0 0 9} \\
9.664 \\
\end{array}$ & $31.88 \%$ & 662 \\
\hline Seve & $\begin{array}{l}\mathbf{0 . 6 4 3} \\
6.569 \\
\end{array}$ & $\begin{array}{c}\mathbf{0 . 9 7 5} \\
57.962 \\
\end{array}$ & $\begin{array}{c}-1.960 \\
-10.538 \\
\end{array}$ & $\begin{array}{c}\mathbf{0 . 0 2 0} \\
14.312 \\
\end{array}$ & $45.73 \%$ & 409 \\
\hline Eight & $\begin{array}{c}\mathbf{0 . 7 2 1} \\
27.048 \\
\end{array}$ & $\begin{array}{c}\mathbf{0 . 9 3 8} \\
34.166 \\
\end{array}$ & $\begin{array}{l}-1.418 \\
-5.275 \\
\end{array}$ & $\begin{array}{l}\mathbf{0 . 0 1 9} \\
9.410 \\
\end{array}$ & $33.83 \%$ & 237 \\
\hline Nine & $\begin{array}{c}\mathbf{0 . 6 9 8} \\
74.403\end{array}$ & $\begin{array}{c}\mathbf{0 . 7 7 7} \\
32.109\end{array}$ & $\begin{array}{l}-3.196 \\
-6.107 \\
\end{array}$ & $\begin{array}{l}\mathbf{0 . 0 3 2} \\
9.438\end{array}$ & $34.89 \%$ & 185 \\
\hline Ten & $\begin{array}{c}\mathbf{0 . 6 9 2} \\
62.237 \\
\end{array}$ & $\begin{array}{c}\mathbf{0 . 6 3 2} \\
19.147 \\
\end{array}$ & $\begin{array}{l}6.642 \\
3.277 \\
\end{array}$ & $\begin{array}{l}-0.026 \\
-2.097 \\
\end{array}$ & $27.10 \%$ & 125 \\
\hline Eleven & $\begin{array}{c}\mathbf{0 . 6 1 1} \\
16.266 \\
\end{array}$ & $\begin{array}{c}\mathbf{0 . 8 7 7} \\
14.867 \\
\end{array}$ & $\begin{array}{c}17.304 \\
6.234 \\
\end{array}$ & $\begin{array}{l}-0.075 \\
-4.480 \\
\end{array}$ & $13.96 \%$ & 83 \\
\hline ve+ & $\begin{array}{c}\mathbf{0 . 6 7 5} \\
13.352\end{array}$ & $\begin{array}{c}\mathbf{0 . 6 0 3} \\
15.149\end{array}$ & $\begin{array}{c}17.368 \\
4.608 \\
\end{array}$ & $\begin{array}{l}-0.078 \\
-3.480 \\
\end{array}$ & $12.77 \%$ & 128 \\
\hline
\end{tabular}

Note: t statistics are in italic 
Table 2-9: Regression Results, Panel Data Random Effects (Non-Spatial Pooled Data)

Dependent Variable: Spot Price

\begin{tabular}{|c|c|c|c|c|c|}
\hline \# Constraints & $\mathbf{F}$ & $T$ & $T^{2}$ & Adj-R ${ }^{2}$ & \# Obs. \\
\hline None & $\begin{array}{c}\mathbf{0 . 8 5} \\
401.95\end{array}$ & $\begin{array}{c}-0.82 \\
-57.33\end{array}$ & $\begin{array}{l}\mathbf{0 . 0 0 8} \\
53.01\end{array}$ & $62.39 \%$ & 10672 \\
\hline One & $\begin{array}{c}\mathbf{0 . 8 2} \\
337.20\end{array}$ & $\begin{array}{c}-0.64 \\
-40.65\end{array}$ & $\begin{array}{l}0.006 \\
41.76\end{array}$ & $52.48 \%$ & 10022 \\
\hline Two & $\begin{array}{c}\mathbf{0 . 8 4} \\
251.17 \\
\end{array}$ & $\begin{array}{c}-0.79 \\
-35.80 \\
\end{array}$ & $\begin{array}{l}\mathbf{0 . 0 0 7} \\
33.15 \\
\end{array}$ & $48.90 \%$ & 6378 \\
\hline Three & $\begin{array}{c}0.85 \\
22.64\end{array}$ & $\begin{array}{c}-0.75 \\
-24.91 \\
\end{array}$ & $\begin{array}{c}\mathbf{0 . 0 0 6} \\
196.11 \\
\end{array}$ & $48.03 \%$ & 3945 \\
\hline Four & $\begin{array}{c}\mathbf{0 . 9 2} \\
147.39\end{array}$ & $\begin{array}{c}-0.72 \\
-16.62\end{array}$ & $\begin{array}{l}\mathbf{0 . 0 0 5} \\
13.51\end{array}$ & $45.35 \%$ & 2371 \\
\hline Five & $\begin{array}{c}\mathbf{0 . 8 8} \\
112.88\end{array}$ & $\begin{array}{c}-0.80 \\
-13.80\end{array}$ & $\begin{array}{l}\mathbf{0 . 0 0 6} \\
11.15\end{array}$ & $47.18 \%$ & 1311 \\
\hline Six & \begin{tabular}{|c|}
$\mathbf{0 . 8 8}$ \\
72.72 \\
\end{tabular} & $\begin{array}{c}-1.15 \\
-11.87 \\
\end{array}$ & $\begin{array}{l}\mathbf{0 . 0 0 9} \\
10.71 \\
\end{array}$ & $42.81 \%$ & 662 \\
\hline Seven & $\begin{array}{c}\mathbf{0 . 8 5} \\
63.08 \\
\end{array}$ & $\begin{array}{c}-1.67 \\
-11.67 \\
\end{array}$ & $\begin{array}{l}\mathbf{0 . 0 1 4} \\
11.71 \\
\end{array}$ & $48.66 \%$ & 409 \\
\hline Eight & $\begin{array}{c}\mathbf{0 . 8 6} \\
32.49\end{array}$ & $\begin{array}{l}-2.38 \\
-9.75\end{array}$ & $\begin{array}{l}\mathbf{0 . 0 2 4} \\
10.73\end{array}$ & $36.19 \%$ & 237 \\
\hline Nine & $\begin{array}{c}\mathbf{0 . 7 8} \\
34.97\end{array}$ & $\begin{array}{l}-3.05 \\
-9.48\end{array}$ & $\begin{array}{c}\mathbf{0 . 0 2 3} \\
9.50\end{array}$ & $39.08 \%$ & 185 \\
\hline Ten & $\begin{array}{c}0.72 \\
20.78\end{array}$ & $\begin{array}{c}-21.42 \\
-6.13\end{array}$ & $\begin{array}{c}\mathbf{0 . 1 4 0} \\
6.63\end{array}$ & $30.71 \%$ & 125 \\
\hline Eleven & $\begin{array}{c}\mathbf{0 . 8 8} \\
10.43 \\
\end{array}$ & $\begin{array}{c}-37.87 \\
-4.46 \\
\end{array}$ & $\begin{array}{c}\mathbf{0 . 2 4 3} \\
4.80 \\
\end{array}$ & $18.55 \%$ & 83 \\
\hline Twelve+ & $\begin{array}{c}\mathbf{0 . 5 8} \\
18.57\end{array}$ & $\begin{array}{l}5.63 \\
1.20\end{array}$ & $\begin{array}{r}-0.027 \\
-0.97\end{array}$ & $22.29 \%$ & 128 \\
\hline
\end{tabular}

Note: $t$ statistics are in italic 
Figure 2-1: Seasonal Cycles for Real-Time and Day-Ahead Prices
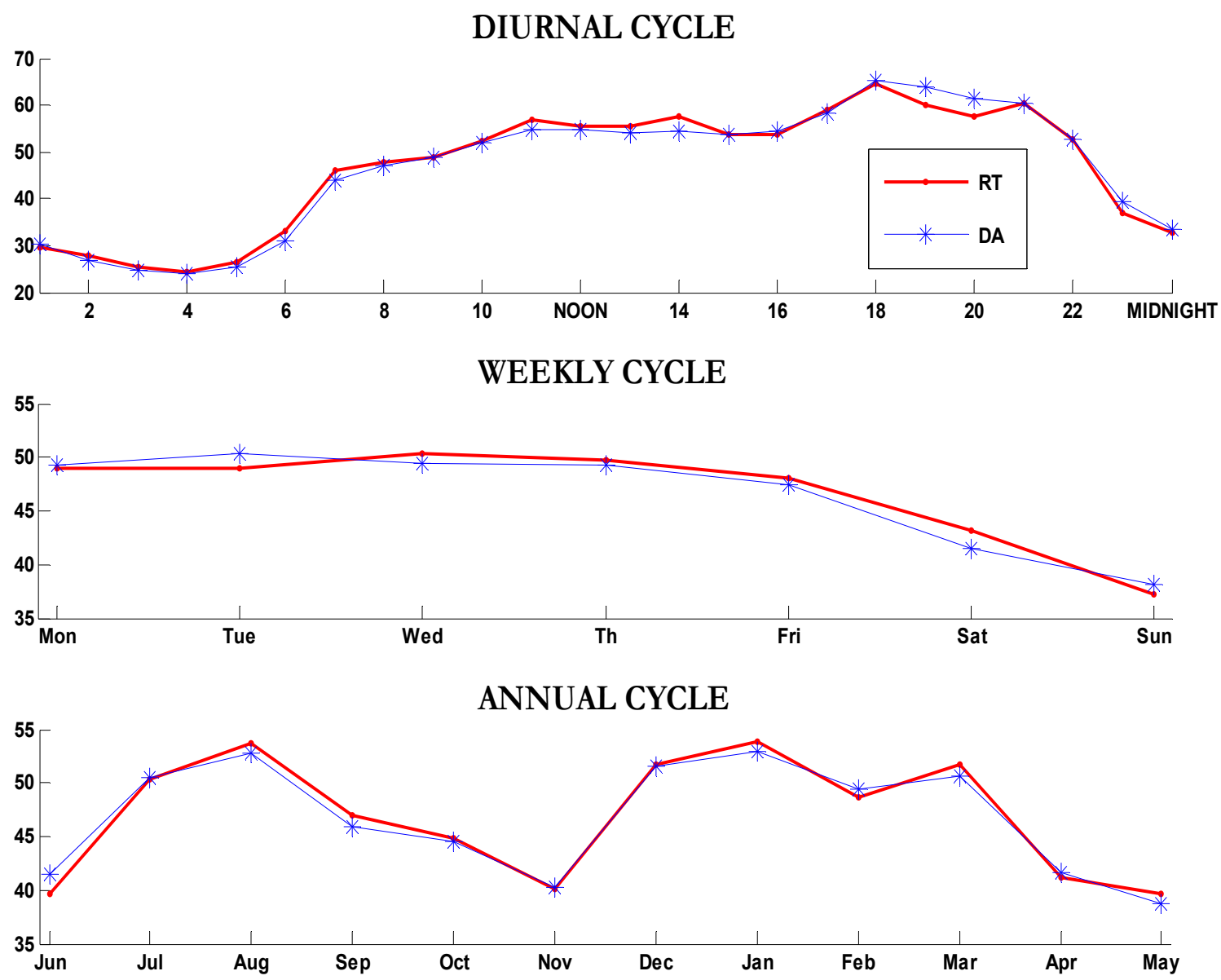
Figure 2-2: Estimated Spatial Coefficient for $\rho$ Electrical Contiguity Weight Matrix

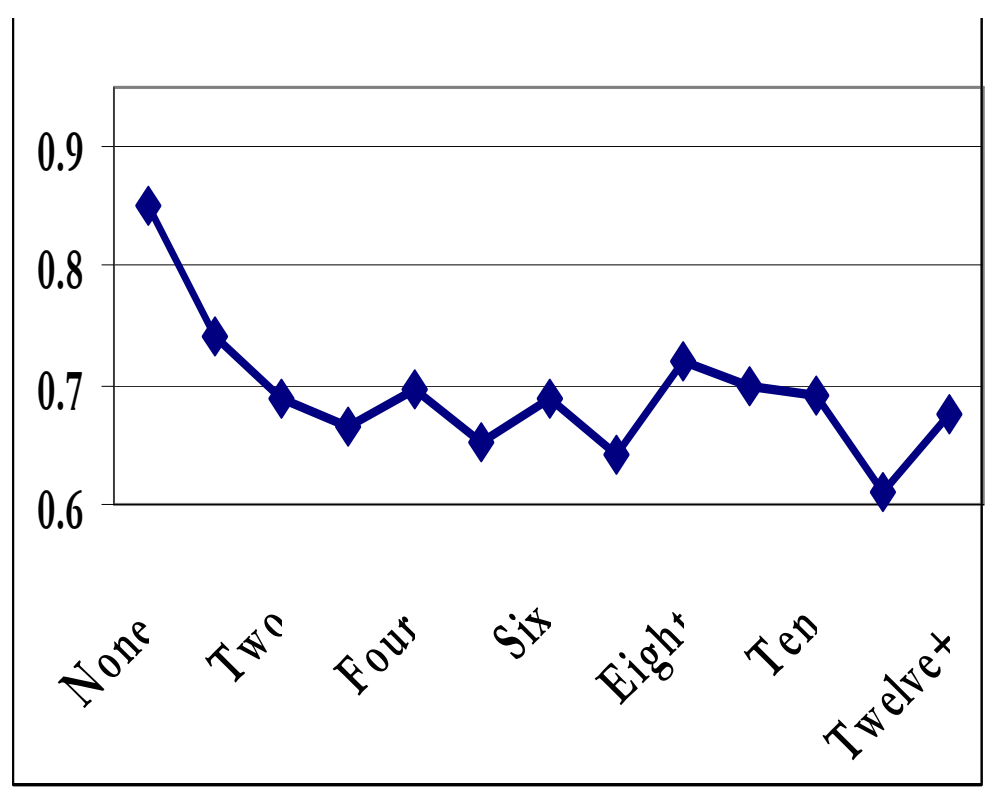

Figure 2-3: Estimated Spatial Coefficient $\rho$ for Geographical Contiguity Weight Matrix

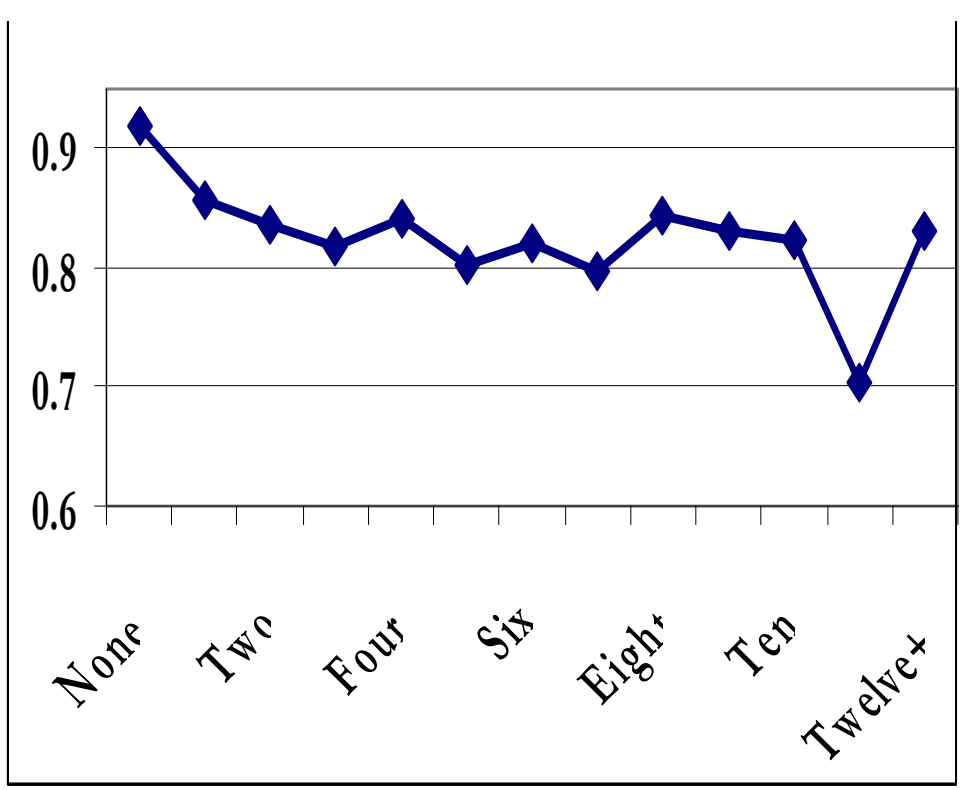




\section{Chapter 3 : Storage and the Electricity Forward Premium}

\section{Introduction}

The classical theory of pricing for commodity forward contracts relies on traders' ability to arbitrage the forward contract by purchasing and storing the commodity (e.g., Working 1948; Kaldor 1939). Electricity storage is too expensive and limited for effective arbitrage, so the current theory of electricity forward contract pricing relies instead upon a model of the supply and demand of hedges by risk-averse electricity producers and retail load serving entities (Bessembinder and Lemmon, 2002). Because, however, power plant fuels may be stored (as well as power plant capacity reserved), arbitrage and conversion opportunities may still place limits upon electricity forward prices. We find empirical evidence that in fact the electricity forward premium is limited by the availability of gas storage inventories, but only in times of relatively high demand for electricity and low space-heating demand for gas.

Our empirical model provides a better fit for the premium and a better test of current theory than is found in the literature to date. The model provides a theoretical basis for practitioners who are seeking to model and predict the forward premium in real time, and it has implications for the prediction of electricity price spikes and the valuation of gas storage facilities. The paper is organized into four sections, as follows. Following this introduction, section II provides a brief description of electricity markets and gas storage. Section III provides an analysis of the current theoretical literature on this subject and the theoretical foundation for our empirical work. Section IV describes our empirical model and discusses the expected signs of the coefficient estimates. Section V describes the data we used and our empirical results. Section VI concludes, and suggests some directions for future research.

\section{Background}


Wholesale electricity markets have undergone rapid and sometimes rocky development worldwide over the past fifteen or twenty years. There are three broad classes of participants in markets for electrical energy: wholesale-market generators, retail-market final consumers, and the load-serving entities (LSEs) that link the two by purchasing in the wholesale market and selling in the retail market. (Traditional electric utilities integrate the generation and marketing functions within a single firm.) In the United States, retail electricity markets have time-invariant prices that are heavily regulated by state authorities, while wholesale electricity prices are set in regional markets, are lightly regulated by the Federal government, and vary rapidly and significantly on a daily pattern.

Wholesale electricity prices are characterized by positive skewness, heteroskedasticity with volatility clustering, and mean reversion. Price spikes are a major issue in electricity markets. Dramatic spikes are relatively common in electricity markets because demand is highly inelastic and subject to sudden shifts, the supply of electricity is also highly inelastic when production is near capacity, and storage of electricity is very expensive. Price spikes occur when demand outstrips supply, which may happen because of an extreme demand shift, equipment failure, transmission congestion, or strategic withholding of capacity by generators. Wholesale price spikes are the biggest source of market risk for LSEs because LSEs purchase power on the wholesale market at a price that may be orders of magnitude higher than the fixed, regulated price at which they sell it on the retail market. The potentially devastating effects of this wholesale/retail market price variability mismatch for LSEs were demonstrated forcefully during the California market crisis in 2000-01. Generators, on the other hand, face their greatest risk of loss when prices and consumption fall, largely because they incur significant costs when they must shut down and restart baseload power plants.

In most wholesale power markets a day-ahead forward market is available to aid participants in planning, price discovery, and hedging. Electricity forward market prices are less volatile than real-time spot market prices, but their distributions are also skewed and 
heteroskedastic. The day-ahead forward price for delivery on day $t$, designated $F_{t}$, need not be an unbiased predictor of the future spot price $\left(S_{t}\right)$ at time $t$, particularly when market participants are risk-averse. Therefore, the forward premium, defined as the excess of the forward price over the expected spot price, Premium $_{t}=F_{t}-\mathrm{E}\left[S_{t}\right]$, may differ systematically from zero.

Classically, the theory of the forward premium begins with the observation that a physical hedge is available for the forward contract; specifically, the flows (of cash and commodity) from the purchase of a forward contract can be duplicated by purchasing the commodity on the spot market at time t-1 and storing it until time t. The existence of this physical hedge provides an arbitrage opportunity that limits the variability of the forward price of storable commodities. Unfortunately, the high expense and technical inefficiency of electricity storage methods preclude construction of a physical electricity hedge of the kind contemplated in classical forward-pricing theory.

Although electricity can not be stored economically, natural gas can be stored in salt caverns, aquifers, and depleted gas wells. In the United States, natural gas storage is owned by pipelines, local gas distribution companies (LDCs), and independent storage operators. See EIA (2004, 2007a, 2007b) and NPC (2003) for information and analysis of North American natural gas storage. In the mid-Atlantic region that is covered by the empirical section of this paper there is an extensive network of natural gas storage facilities consisting mostly of depleted gas wells linked by pipelines. This network serves the sometimes conflicting needs of space heating for residential and commercial consumers, industrial chemical and fertilizer plants, and gas-fired power plants. Although the three sectors are nearly equal in their consumption, for obvious reasons space heating applications traditionally take precedence over other applications during cold weather (EIA 2006).

Despite their historical role as appendages of the gas pipelines and local distribution companies, since the implementation of "open access" under FERC Order 636 (1992) gas storage facilities increasingly operate in response to market signals. EIA $(2004$, p. 9) asserts,

"Open access has allowed storage to be used other than simply as backup inventory or a supplemental seasonal supply source. For example, marketers and other third parties may move 
gas into and out of storage. . . as changes in price levels present arbitrage opportunities. Further, storage is used in conjunction with various financial instruments ... in ever more creative and complex ways in an attempt to profit from market conditions."

Most of these changes have been driven by the growing importance of gas-fired electricity generation. Since 1992 gas-fired electricity generation capacity has more than doubled, and gas consumption by electricity generation has grown from $17 \%$ to $29 \%$ of total U.S. natural gas consumption (EIA 2006, 2007). Because gas-fired turbines produce relatively little pollution, and are quick and easy to start and ramp up, they are often the technology of choice for load-following and peak generation. Supplying the electric power industry is therefore an increasingly important aspect of the natural gas business, and the supply of natural gas is increasingly crucial to the efficient operation of the electric power industry, particularly during peak and shoulder hours.

Broadly, natural gas storage is filled during the spring, fall, and summer, and emptied during the winter. Net injections into storage occur over the daily cycle, even during the peak summer electricity demand months of July and August. However, according to NPC (2003), the "summer [electricity demand] peak impacts the summer season gas storage injection period, primarily allowing for injections only in the off-peak electric demand hours of the day. . . ." Thus, because of the diurnal cyclicity of electricity hourly demand there is some threat of hourly pipeline constraints even in summer months.

\section{Theory}

Because the cost of fuel composes most of the marginal cost of electricity, and gas is often the marginal fuel, a limited hedge for electricity prices may be constructed by purchasing and storing natural gas. The effectiveness of this hedge depends on the availability of generation capacity, emission rights, and transmission capacity needed to convert stored gas to electricity and deliver the power when and where it is needed. Because of these operational difficulties, current models of the electricity forward premium ignore the effect of stored natural gas. Still, the lack of available natural gas in storage can increase the price in thin natural gas spot markets (Borenstein 
et al, 2007). It can also increase the probability of a shortage of power availability from gas-fired plants, which will increase the probability of an electricity price spike. The availability of gas storage inventories therefore affects the day-ahead perception of the distribution of spot electricity prices, which will affect the forward premium if buyers and sellers are risk-averse. We describe the theory behind this two-stage process below, taking the second stage first.

\section{III.1 How the Distribution of Electricity Spot Prices Affects the Future Premium}

The effect of the anticipated distribution of electricity prices on the forward premium is relatively well-established in the Finance literature. The leading paper on the subject, Bessembinder and Lemmon (2002), hereafter referred to simply as "BL," derives the premium in a model of risk-averse generation firms who sell electricity, and risk-averse LSEs who buy electricity, in a wholesale spot market. Both parties hedge their risk in the day-ahead forward market. The generators and LSEs use the forward contracts as hedges to help maximize objective functions that take into account both profit and uncertainty. Generators (who are ordinarily net suppliers of forward contracts) seek to minimize their exposure to low prices or demand shortfalls, and also seek to stabilize their revenues generally. LSEs (who are ordinarily net purchasers of forward contracts) seek to reduce their exposure to low sales and stabilize their procurement costs, and they especially want to hedge their exposure to price spikes.

In BL's model, the forward premium rises and falls to maintain equilibrium between supply and demand for forward contracts. The possibility of price spikes creates positive skewness in the perceived distribution of the electricity spot price. To avoid losses, LSEs react to increased spot price skewness by demanding more forward contracts, driving the forward price upward relative to the expected spot price and increasing the forward premium (BL Hypothesis 2, page 1362). Electricity sales and the wholesale price are positively correlated, and this positive correlation creates a positive profit exposure for LSEs as long as the retail price of electricity exceeds the expected spot price. Thus, when the expected spot price is below the retail price (which should, on 
average, be the case) an increase in spot price variance will reduce LSEs' net downside risk, and thereby reduce their demand for forward contracts. There should, therefore, be a negative relationship between the variance of the spot price and the forward premium (BL Hypothesis 1, p.1362).

The overall relationship between the moments of the spot price distribution and the forward premium is summarized by equation (1) (from BL equation 13, page 1358):

$$
\operatorname{Prem}_{t}=\alpha \mathrm{E}\left[\operatorname{Var}\left(S_{t}\right)\right]+\gamma \mathrm{E}\left[\operatorname{Skew}\left(S_{t}\right)\right]
$$

where $\alpha<0$ and $\gamma>0$ are signed parameters in the model. Both parameters increase in absolute value with risk aversion and with the convexity of the production cost function.

A secondary result obtained in the BL model, and emphasized in Ullrich (2006), is that the coefficients $\alpha$ and $\gamma$ in equation (1) decrease in absolute value as the wholesale spot price of electricity increases relative to the fixed retail price of electricity. Expressions in BL (p. 1359) indicate

$$
\alpha=\Omega E\left[S_{t}\right]^{x-1}\left(E\left[S_{t}\right]-P_{R}\right) \text { and } \gamma=\frac{1}{2} \Omega x E\left[S_{t}\right]^{x-2}\left\{x E\left[S_{t}\right]-(x-1) P_{R}\right\}
$$

where $P_{R}$ is the retail price of electricity, $\Omega$ is a parameter that does not depend on prices, and $x$ is a parameter whose value increases as the convexity of the production function decreases. $\mathrm{BL}$ assert on theoretical grounds that $0<x<1$, and they estimate its value to be about 0.33 .

\section{III.2 How Gas Storage Inventories Affect the Distribution of Electricity Prices}

The BL model as summarized in equations (1) and (2) does not address the question of what factors cause the variance and skewness of the anticipated distribution of the spot price to vary, but both common sense and the literature give ample reason to believe that the moments of the electricity price distribution are affected by the availability of gas storage. Skewness in the distribution of electricity spot prices is driven primarily by the potential for price spikes. Price spikes 
arise from the "hockey-stick" or backward-L shape of the electricity supply curve, together with the volatility and near-perfect inelasticity of electricity demand. When demand peaks (or supply falls sufficiently), the near-vertical demand curve reaches or exceeds the near-vertical segment of the supply curve, and small changes in demand give rise to orders-of-magnitude changes in price.

Gas-fired turbines occupy a crucial location at the "bend" in the hockey-stick electricity generation supply curve. At current gas prices, efficient combined-cycle (CC) gas-fired plants occupy the high shoulder segment of the load curve, and combustion turbines (CT) dominate the segment above that. As the gas-fired CCs and CTs reach capacity prices spike upward, driven by the cost of diesel generators and the value of lost load. As constraints on the potential gas supply increase, price spikes become more likely, which will skew the distribution of the electricity spot price.

A highly simplified example illustrates the point. Suppose hourly demand follows a uniform distribution, normalized to fall between 0 and 1 (i.e., $Q^{D} \sim U(0,1)$ ), so that the probability $\operatorname{Pr}\left(Q^{D}<q\right)=$ $q$ for $0<q<1$. For simplicity, assume that the electricity market is competitive, so that price equals marginal cost. On the supply side, there are three types of plants with three levels of costs, dispatched in merit order. Baseload generators have marginal cost $P_{0}$ and provide generation capacity equal to $\pi_{0}$, so $\operatorname{Pr}\left(P=P_{0}\right)=\pi_{0}$. Gas-fired turbines have higher marginal cost $P_{1}$ and provide generation capacity equal to $\pi_{1}$, so they are the marginal producers whenever $\pi_{0}<Q^{D}<\pi_{0}+\pi_{1}$; hence $\operatorname{Pr}\left(P=P_{1}\right)=\pi_{1}$. The highest-cost resources (e.g. diesel generators and demand response) serve the rest of the load $\left(\pi_{2}=1-\pi_{0}-\pi_{1}\right)$ at a marginal cost of $P_{2}$. Thus, the electricity price follows a trinomial distribution, with prices $P_{0}, P_{1}$, and $P_{2}$ occurring with probabilities $\pi_{0}, \pi_{1}$, and $\pi_{2}=$ $1-\pi_{0}-\pi_{1}$, respectively.

Available gas-fired generation, $\pi_{1}$, will fall when there is a binding constraint on the supply of natural gas, as when storage stocks out or is required for space heating. As viewed from one day ahead, a low level of gas in storage will decrease the probability $\pi_{1}$ that any given unit of 
electricity will cost $P_{1}$, and increase the probability that it will cost $P_{2}$. As $\pi_{1}$ falls, the probability $\pi_{2}$ of a price spike increases, which will increase the skewness and variance of the electricity price distribution. Figure 3-1 shows the effect of natural gas storage constraints on the skewness of the distribution of electricity prices in a simulation in which $P_{0}=1, P_{1}=5, P_{2}=10, \pi_{0}=0.7$, and $\pi_{1}$ falls from 0.3 to 0 while $\pi_{2}$ rises from 0 to 0.3 .

A paper by Routledge, Seppi, and Spatt (2001), hereafter "RSS," uses a relatively elaborate general equilibrium model to obtain the same insight about the effect of gas storage constraints on the price of electricity. The RSS model, an extension of Routledge, Seppi, and Spatt (2000), captures cross-commodity price and physical conversion linkages, including the conversion of natural gas to electricity. Prices of inputs, outputs, and input substitutes are linked through the chain of production and by prices set in competitive markets, but the prices become unlinked in the RSS model when constraints on conversion or storage bind. Like the simple simulation here, the RSS model's key result is that symmetric demand shocks to the electricity market can generate an asymmetrical distribution of electricity prices because of the shape of the supply curve, and that the asymmetry can increase when there are constraints on access to natural gas storage inventories. For tractability in a general equilibrium setting, RSS assume that agents are risk-neutral, but as the simulation above illustrates, risk-neutrality is not required to generate the testable hypothesis that natural gas storage constraints increase electricity spot price skewness.

Our simulation also illustrates RSS's result that constraints upon the supply of natural gas will affect the electricity price only if the marginal plant would be gas-fired in the absence of the constraint. Thus, demand must be greater than $\pi_{0}$ and less than the unconstrained $\pi_{1}$ in our model for the reduction in $\pi_{1}$ to affect the price. Electricity price skewness will increase when temperatures are low because increased space-heating demand for gas makes gas storage inventories unavailable to electricity producers. However, RSS note that the impact of storage inventory fluctuations on electricity price skewness should be small when temperatures are low because the inventory gas would not have been used to generate electricity anyway. 
To summarize, according to our model and RSS an increase in demand or decrease in supply of electricity will positively skew the electricity price distribution, especially if electricity demand is unusually high. An increase in gas storage inventories will reduce the skewness of the electricity price distribution if gas is the marginal fuel for electricity production and the space-heating demand for gas is low. The BL model finds that the forward premium increases with the positive skewness of the electricity distribution, and decreases as the variance of the electricity spot price increases if the expected wholesale spot price is less than the retail price. The marginal effects of variance and skewness will both diminish as the expected spot price rises, according to BL. The combined model therefore predicts that: (1) the effect of a change in electricity demand on the future premium will rise as demand rises; (2) an increase in gas storage inventories will decrease the electricity forward premium if gas is the marginal fuel and the space-heating demand for gas is low, and (3) the marginal effects of skewness and variance will both fall as the expected spot price rises.

\section{Empirical Model}

The current paper is the first to provide an empirical test of these predictions. RSS do not provide any empirical tests of their model, nor do any empirical tests of their model appear elsewhere in the literature, so far as we are aware. BL provide simulation results, but no empirical results, to support the expected signs of the parameters $\alpha$ and $\gamma$ in equation (1). Longstaff and Wang (2004) provide some empirical support for the BL model, but their results are based on a regression that employs only 24 observations (one for each hour of the day). A working paper by Ullrich (2006) refines the BL model and provides some simulations and empirical estimates of the production function's convexity, but does not estimate $\alpha$ or $\gamma$.

\section{Table 3-1 Predicted Effects of Variables on Electricity Price Moments and the Forward Premium}




\begin{tabular}{|c|c|c|c|c|}
\hline Variable & Description & $\begin{array}{l}\text { Effect on } \\
\text { Variance }\end{array}$ & $\begin{array}{l}\text { Effect on } \\
\text { Skewness (RSS) }\end{array}$ & $\begin{array}{l}\text { Effect on } \\
\text { Premium (BL) }\end{array}$ \\
\hline $\operatorname{Var}_{t-1}(S)$ & $\begin{array}{l}\text { Recent Variance of } \\
\text { Spot Price }\end{array}$ & + & 0 & \\
\hline $\operatorname{Skew}_{t-1}(S)$ & $\begin{array}{l}\text { Recent Skewness of } \\
\text { Spot Price }\end{array}$ & 0 & + & + \\
\hline $\mathrm{CDH}_{t}$ & Cooling Degree Hours & + or 0 & ++ for large $\mathrm{CDH}$ & + for large $\mathrm{CDH}$ \\
\hline $\mathrm{HDH}_{t}$ & Heating Degree Hours & + or 0 & + or 0 & + or 0 \\
\hline $\mathrm{GS}_{t-1}$ & $\begin{array}{l}\text { Gas Storage } \\
\text { Inventories }\end{array}$ & $?$ & or 0 & or 0 \\
\hline $\mathrm{CDH}_{t}^{*} \mathrm{GS}_{t-1}$ & $\begin{array}{l}\text { Interaction of Storage } \\
\text { and CDH }\end{array}$ & $?$ & Largẽ & Largé \\
\hline $\mathrm{HDH}_{t}^{*} \mathrm{GS}_{t-1}$ & $\begin{array}{l}\text { Interaction of Storage } \\
\text { and } \mathrm{HDH}\end{array}$ & $?$ & Smali or 0 & Smali or 0 \\
\hline \multicolumn{5}{|c|}{$\begin{array}{l}\text { Effects of } \mathrm{Var}_{t-1} \text { and } \mathrm{Skew}_{t-1} \text { on the premium will decline in absolute value as } \mathrm{E}[\mathrm{S}] \text { rises } \\
\text { ** The predicted partial derivative } \partial \text { Premium/ } \partial \mathrm{GS}_{t-1}<0 \text { only if gas is the marginal fuel and } \\
\mathrm{HDH} \text { is low. }\end{array}$} \\
\hline
\end{tabular}

$$
\begin{aligned}
\text { Premium }_{t}=\beta_{0}+\beta_{1} \operatorname{Var}_{t-1}(\mathrm{~S})+\beta_{2} \text { Skew }_{t-1}(\mathrm{~S})+\beta_{3} G S_{t-1} \\
\\
+\beta_{4 a} C D H_{t}+\beta_{4 b} C D H_{t}^{2}+\beta_{5 a} H D H_{t}+\beta_{5 b} H D H_{t}^{2} \\
+\beta_{6} G S_{t-1}{ }^{*} C D H_{t}+\beta_{7} \mathrm{GS}_{t-1}{ }^{*} H D H_{t}+\varepsilon_{t}
\end{aligned}
$$

Our empirical model, along with the expected signs of derivatives, is summarized in equation (3) and Table 3-1. In our empirical work we do not attempt to model transmission or generation outages, or any other factors that are not related to weather or gas inventories. Instead, we assume that agents will derive some information about the moments of the future distribution of electricity prices by observing the moments of its immediate past distribution, $\operatorname{Var}_{t-1}$ and $S k e w_{t-1}$. Our measures of the second and third central moments of the expected spot price distribution $\left(\mathrm{Var}_{t}\right.$ ${ }_{1}(S)$ and $S_{k e w}(S)$ in equation 3$)$ are calculated for each hourly observation using the previous week's spot price data from the same hour. Thus, $E_{t-1}\left(S_{t}\right)=\frac{1}{7} \sum_{i=2}^{8} S_{t-i} ; \operatorname{Var}_{t-1}=\hat{\sigma}^{2}=$ $\frac{1}{6} \sum_{i=2}^{8}\left(S_{t-i}-E_{t-1}\left(S_{t}\right)\right)^{2}$, and $S_{k e w}=\frac{1}{6} \sum_{i=2}^{8}\left(S_{t-i}-E_{t-1}\left(S_{t}\right)\right)^{3}$, where $S_{t-i}$ is the spot price from the same hour, $i$ days in the past.

Including backward-looking measures of the moments of the spot price distribution helps control for changes in perceptions that occur for reasons (such as equipment outages) that are more persistent than weather-related shifts. Thus, the empirical parameters $\beta_{1}$ and $\beta_{2}$ in equation (3) differ from the theoretical parameters $\alpha$ and $\gamma$ in equations (1) and (2), which measure the effect 
of all information about the distribution of $S_{t}$ available in time $t-1$, including the weather forecast for time $t$. The effect of the backward-looking empirical moments should be smaller, during peak demand hours, when forward-looking factors (in particular the temperature forecast) are more relevant to day-ahead expectation formation. Hence, the empirical parameters $\beta_{1}$ and $\beta_{2}$ should drift nearer to zero during peak demand hours, reinforcing the theoretical relationship shown in equation (2).

The temperature is the biggest single determinant of the position of the electricity demand curve, but its effect depends on several factors. High temperatures increase the demand for electricity, as air conditioning is almost entirely powered by electricity. Cold temperatures increase gas demand for space heating, but they have less of an effect on the demand for electricity because electricity does not dominate the heating market. Because of these asymmetrical effects of high and low temperatures on electricity demand and gas inventory availability, in our empirical model we measure temperature using separate variables for Heating Degree Hours $(\mathrm{HDH})=$ $\max \{0$, Temperature -65$\}$ and Cooling Degree Hours $(C D H)=\max \{0,65-$ Temperature $\}$ In the data, the $\mathrm{CDH}$ variable is always zero when $\mathrm{HDH}$ is positive, and vice versa, and temperatures are measured in degrees Fahrenheit.

Periods of high heating and cooling load (and particularly the latter) coincide with the periods of greatest risk of price spikes, and therefore the greatest skewness of the anticipated electricity price distribution. The theoretical predictions summarized in Table 3-1 therefore indicate nonnegative effects of $\mathrm{HDH}$ and $\mathrm{CDH}$ on spot price variance and skewness and the forward premium, and that $\mathrm{HDH}$ will have a smaller effect than $\mathrm{CDH}$. To allow for nonlinear effects, we use a quadratic form (see Engel et al, 1986) because the shape of the supply curve suggests a positive second derivative, so we expect $\beta_{4 b}>\beta_{5 b}>0$.

Gas storage inventory availability mitigates the risk of electricity price spikes, which, according to $\mathrm{BL}$, reduces the forward premium. As discussed above and emphasized in RSS, however, inventories can affect the electricity spot price distribution only if stored gas would fuel 
marginal generators, and only if the gas inventories are not required for space heating - i.e., only when electricity demand is high and gas demand for other purposes is not high. Therefore, the effect of gas storage inventories $G_{t-1}$ on the moments of the electricity spot price and on the forward premium will vary with temperature, and the partial derivative

$$
\partial \text { Premium }_{t} / \partial \mathrm{GS}_{t-1}=\beta_{3}+\beta_{6} \mathrm{CDH}_{t}+\beta_{7} \mathrm{HDH}_{t} \leq 0
$$

will be non-positive. The derivative $\partial$ Premium $_{t} / \partial G S_{t-1}$ should be strictly negative when electricity demand for cooling is high and space-heating demand for gas is low $(\mathrm{CDH}>0, \mathrm{HDH}=0)$. The derivative will also be negative when electricity demand for heating $(\mathrm{HDH})$ is high, unless gas heating demand precludes the use of gas storage inventories for electricity generation. Thus, our model predicts $\beta_{6}<0, \beta_{7} \leq 0$, and $\left|\beta_{6}\right|>\left|\beta_{7}\right|$. The sign of $\beta_{3}$ will depend on the data values, but overall $\partial$ Premium $_{t} / \partial \mathrm{GS}_{t-1} \leq 0$. By the same token, $\beta_{6}<0$ implies that $\partial$ Premium $_{t} / \partial C D H_{t}=\beta_{4 a}+$ $2 \beta_{4 b} C D H_{t}+\beta_{6} \mathrm{GS}_{t-1}$ will rise as gas storage inventories fall.

The predictions of our model with respect to the interaction coefficients $\beta_{6}$ and $\beta_{7}$ provide the central testable hypotheses in our research. The estimate of $\beta_{6}$ should be greater than the estimate of $\beta_{7}$ in absolute value for at least three reasons. First, the value of gas for space heating is greater when $\mathrm{HDH}$ is high, so gas storage inventories may not be available to serve electricity production when $\mathrm{HDH}$ is high. Second, because our data are from the PJM system, which peaks in the summer, the skewness of peak-summer prices is greater than the skewness of peak-winter prices. Therefore the scope for skewness reduction is greater when $\mathrm{CDH}$ is high than when $\mathrm{HDH}$ is high, implying a relatively greater marginal effect of gas storage on skewness when $\mathrm{CDH}$ is high. Third, as Ullrich (2006) emphasizes, when high expected CDH drives the expected wholesale spot price above the retail price the sign of the coefficient on expected variance $\alpha$ in equation (1) flips from negative to positive. Therefore, on very hot days available gas storage inventories will reduce 
the premium by reducing both variance and skewness, which will further increase the magnitude of $\beta_{7}$

\section{Data and Estimates}

\section{V.1 Data}

We estimate the empirical model in equation (3) using market data from the PJM Interconnection. PJM is a regional transmission organization established in 1997 as the first auction-based electricity market in the U.S., and the largest wholesale power market in the world. It coordinates the continuous buying and selling of energy in real-time spot and day-ahead forward markets. PJM electricity prices use a locational marginal-cost pricing (LMP) methodology, which means that they reflect not only generation supply and demand, but also physical constraints and other characteristics of the system.

Since June 1, 2000 PJM has offered two types of electricity markets. The day-ahead market is a forward market in which hourly prices are calculated for the next operating day based on demand bids, and generation supply offers and bids. By 4:00 PM each day, the PJM announces the 24 clearing prices, production schedules, and trades for the next day's operation. In the next day's real-time spot market PJM runs an electronic auction by matching bids and offers, determining market-clearing prices every five minutes. Transactions are settled hourly for both real time and day-ahead markets.

The primary data set for this study consists of four years of hourly real-time spot prices and hourly day-ahead forward prices from January 1, 2001 to December 31, 2004. For each of the 1461 days in the total sample, we have information on the PJM market average spot and forward prices (quoted in dollars per megawatt hour, $\$ / M W h$ ) for each of the 24 hours. The market average prices are load-weighted averages over all of the nodes in the PJM Interconnection, and were downloaded from the PJM website www.pjm.com. Because the coefficients in the model can be expected to change throughout the day, the data set is divided into 24 separate time series, and 
separate regressions are estimated for each hour. Separate treatment of each hour is common in the literature (e.g., Ramanathan et al, 1997, Fay et al, 2003, Longstaff and Wang, 2004), allows us to finesse the issue of controlling for intraday price fluctuations, and allows us to examine how the regression coefficients change during the day.

Table 3-2, Table 3-3, and Table 3-4 display summary statistics for electricity spot and forward prices, and forward premia for each hour. Both spot and forward prices display significant positive skewness and leptokurtosis (fat tails), reflecting the incidence of price spikes. Electricity price kurtosis, skewness, variance, and mean all vary in a daily cycle, and all are much higher during the afternoon peak hours. Skewness is positive for both series and all hours. Forward prices have lower variance, skewness, and kurtosis than spot prices, although their means are nearly equal. The forward premium is much less skewed (it is in fact negatively skewed during afternoon hours) and less leptokurtic than either price distribution. The mean and median forward premium are both slightly negative in most hours, but the mean is less than 0.2 standard errors from zero in all cases.

We use weekly gas storage data on the Eastern Consumption Region from the Energy Information Administration. Before March 15, 2002, the storage data are estimated by the EIA based on both EIA monthly survey data and AGA weekly survey data; after that date, they are from the EIA weekly survey, EIA-912, "Weekly Underground Natural Gas Storage Report.” Although these storage data are reported at a different frequency than the price data (weekly versus daily), we use them because (1) they are the only data available, and (2) they are reasonably accurate. Because the stock amount in storage is very large relative to the daily flow of injections and withdrawals, and weekly inventory changes are on the order of two to four percent, the amounts in storage do not change significantly from day to day. For a robustness check, we estimated the regressions using smoothed and interpolated gas storage data, and the regression results were qualitatively the same. 
Heating Degree Hour (HDH) and Cooling Degree Hour (CDH) data are hourly averages of 13 weather stations located in the PJM Interconnection. They are taken from the Global Summary of the Day (GSOD) database archived by the National Climatic Data Center (NCDC), and compiled and cleaned by researchers at ZedX, Inc, Atmospheric Sciences Division. Note that hourly temperature in degrees Fahrenheit equals $(65-\mathrm{HDH})$ when $\mathrm{HDH}>0$, and it equals $(65+\mathrm{CDH})$ when $\mathrm{CDH}>0$.

\section{V.2 Regression Results}

We estimate equation (3) using ordinary least squares and report the results in Table 3-5,

Table 3-6 Table 3-7, and Table 3-8. Hypothesis tests use the Newey-West covariance matrix, which is robust to heteroskedasticity and serial correlation. Adjusted R2 statistics suggest a reasonably good fit of the regression over the entire period and for most hours, particularly during the crucial afternoon and early hours when price spikes become more likely and our model is therefore more relevant.

Regression results generally support the model's important predictions as to coefficient sign, daily pattern, and statistical significance. For example, as figure 2 illustrates, the estimated effect of increased recent spot price variance is negative and statistically significant in almost all hours, and the effect of increased recent spot price skewness is generally positive. Both the coefficient estimates for $\beta_{1}$ and $\beta_{2}$ show the expected daily cyclical pattern, falling in magnitude in the afternoon as the expected spot price rises toward the level of the retail price.

Figure 3-3 shows that the estimated effect of temperature on the forward premium $(2$ Premium/ $/ 2 C D H)$ is positive for most hours of the day and for temperatures above 75 degrees Fahrenheit $(\mathrm{CDH}>10)$. The marginal effect of $\mathrm{CDH}$ on the premium increases rapidly as the temperature rises, as measured by the positive and significant coefficients on $\mathrm{CDH}^{2}$ in Table 3-5 and. The effect of higher temperatures (increased $\mathrm{CDH}$ ) rises in the afternoon hours as the demand curve approaches the convex portion of the supply curve. The marginal effect of HDH 
increases with system load as well, but it is much smaller than that of $\mathrm{CDH}$ and does not exhibit a strong daily pattern, all of which is also consistent with the predictions of the model.

The principal novel predictions of the model in this paper, however, concern the effect of natural gas storage inventories on the forward premium. Again, the empirical results strongly support the theoretical predictions. The estimated coefficient of the interaction term between CDH and storage, $\beta_{6}$ in equation (3), is negative in all 24 hours (and is statistically significant in 23 of the 24 hours), supporting the hypothesis that increased gas storage inventories significantly reduce the forward premium. The $\mathrm{HDH}^{*}$ Storage interaction coefficient estimates, $\beta_{7}$ in equation (3), are also negative but much smaller than the $\beta_{6}$ interaction coefficient estimates, supporting predictions of a smaller impact of gas inventories on electricity price skewness when heating demand is high.

Overall, the total effect of gas storage inventories on the electricity forward premium is greater for $\mathrm{CDH}$ than $\mathrm{HDH}$, and increases more steeply with load, regardless of the hour of the day.

Figure 3-3 provides a graphic illustration of the ability of storage to reduce the skewness of the electricity price distribution. Recall that $\beta_{6}<0$ implies that $\partial$ Premium $_{t} / \partial C D H_{t}=\beta_{4 a}+2 \beta_{4 b} C D H_{t}+$ $\beta_{6} \mathrm{GS}_{t-1}$ will rise as gas storage inventories fall. Comparing the left and right panels of figure 3-3, the vertical shift of the surface indicates that the effect of changes in temperature (i.e., CDH) on the forward premium is greater when gas storage inventories are low.

\section{Conclusion}

This paper provides evidence for the proposition that any complete model of the electricity forward premium must include information about natural gas storage inventories. Our model of the effect of gas storage on the electricity forward premium augments the model of Bessembinder and Lemmon (2002) by incorporating insights about the source of electricity price skewness and variance similar to those of Routledge, Seppi, and Spatt (2001). Our empirical results reinforce and confirm the results reported in Longstaff and Wang (2004), while offering a methodological improvement. Furthermore, they go beyond Bessembinder and Lemmon and Longstaff and Wang 
by showing strong support for the proposition that the availability of stored gas has a measurable effect on the electricity forward premium, and that information about gas storage inventories adds information to electricity price analysis beyond the information contained in the temperature data by itself.

Improving our understanding of the electricity forward premium requires improving our understanding of the electricity price spikes that drive that premium. We have provided evidence that gas storage inventories are related to electricity price spikes, described a theory of the nature of that relationship, and tested its empirical implications using data from the largest wholesale power market in the world. As data series from other power markets with significant gas storage (for example, the U.S. Midwest and Europe) lengthen and improve, further tests of the model in this paper will be possible. Because of the financial importance of electricity price spikes, improving our understanding of the role of gas storage inventories in their occurrence should be of value to practitioners. Understanding the link between gas storage and electricity market price risk may also improve our ability to understand and quantify the value of gas storage facilities, both to the markets and to society at large.

Further research is required on practical applications of our model to the models currently in use by utilities, power producers, gas storage managers, and other practitioners. For practical applications to other markets and to the needs of practitioners, the empirical model will require modifications depending upon the availability of information and the characteristics of demand and supply in other electricity markets. Still, the basic principles that follow from our work are fairly clear: the value of gas inventories in reducing electricity price risk depends upon the level of gas demand and the expected spot price of electricity in a predictable way. Because the electricity forward premium is intimately related to the likelihood of electricity price spikes, the explicit incorporation of natural gas storage information into electricity spot and forward market models should improve our ability to forecast electricity price spikes, prices, and forward premia. 
Table 3-2: Summary Statistics for PJM Real-Time Spot Electricity Price

\begin{tabular}{|c|c|c|c|c|c|c|c|c|}
\hline Hour & Min & Max & Mean & Median & $\begin{array}{c}\text { Standard } \\
\text { Deviation } \\
\sigma\end{array}$ & $\begin{array}{c}3^{\text {rd }} \text { Moment } \\
\mu_{3} \mathbf{1 0}^{-4}\end{array}$ & $\begin{array}{c}\text { Skewness } \\
\text { coefficient } \\
\mu_{3} / \sigma^{3}\end{array}$ & Kurtosis \\
\hline $\mathbf{1}$ & 1.29 & 100.12 & 22.23 & 18.61 & 11.65 & 0.37 & 2.31 & 10.95 \\
\hline $\mathbf{2}$ & -5.80 & 128.94 & 21.08 & 17.48 & 12.08 & 0.44 & 2.52 & 13.30 \\
\hline $\mathbf{3}$ & -3.75 & 94.58 & 19.17 & 16.51 & 11.37 & 0.41 & 2.77 & 14.46 \\
\hline $\mathbf{4}$ & -4.62 & 106.33 & 18.53 & 16.10 & 11.11 & 0.42 & 3.06 & 17.68 \\
\hline $\mathbf{5}$ & -1.94 & 125.07 & 19.81 & 16.76 & 12.00 & 0.57 & 3.31 & 18.87 \\
\hline $\mathbf{6}$ & 0.56 & 139.70 & 24.03 & 19.62 & 13.96 & 0.63 & 2.33 & 11.27 \\
\hline $\mathbf{7}$ & 0.93 & 152.13 & 33.21 & 26.70 & 22.31 & 1.92 & 1.73 & 6.81 \\
\hline $\mathbf{8}$ & 0.71 & 166.40 & 35.92 & 28.75 & 23.32 & 2.24 & 1.76 & 7.00 \\
\hline $\mathbf{9}$ & 6.20 & 180.12 & 35.92 & 31.04 & 19.36 & 1.32 & 1.81 & 8.15 \\
\hline $\mathbf{1 0}$ & 13.83 & 142.46 & 39.15 & 35.02 & 19.26 & 1.07 & 1.50 & 6.30 \\
\hline $\mathbf{1 1}$ & 13.72 & 210.99 & 43.54 & 39.81 & 21.68 & 1.56 & 1.54 & 7.80 \\
\hline $\mathbf{1 2}$ & 12.89 & 623.01 & 43.23 & 38.18 & 30.26 & 24.69 & 8.91 & 151.50 \\
\hline $\mathbf{1 3}$ & 12.90 & 916.68 & 42.91 & 36.25 & 40.31 & 87.61 & 13.38 & 262.45 \\
\hline $\mathbf{1 4}$ & 9.42 & 931.43 & 45.73 & 38.61 & 46.50 & 113.28 & 11.27 & 180.20 \\
\hline $\mathbf{1 5}$ & 8.53 & 931.53 & 42.34 & 33.49 & 47.38 & 126.42 & 11.89 & 198.14 \\
\hline $\mathbf{1 6}$ & 10.23 & 931.93 & 41.57 & 32.23 & 45.61 & 109.23 & 11.51 & 187.90 \\
\hline $\mathbf{1 7}$ & 11.83 & 932.27 & 45.58 & 37.83 & 47.54 & 123.36 & 11.48 & 186.88 \\
\hline $\mathbf{1 8}$ & 6.23 & 932.25 & 49.77 & 42.77 & 43.49 & 97.07 & 11.80 & 224.53 \\
\hline $\mathbf{1 9}$ & 6.81 & 692.92 & 45.05 & 38.75 & 31.21 & 21.95 & 7.22 & 131.77 \\
\hline $\mathbf{2 0}$ & 5.49 & 375.45 & 42.47 & 37.64 & 23.95 & 4.07 & 2.96 & 29.87 \\
\hline $\mathbf{2 1}$ & 13.47 & 909.08 & 46.06 & 41.63 & 32.63 & 47.02 & 13.53 & 340.81 \\
\hline $\mathbf{2 2}$ & 13.50 & 325.04 & 39.65 & 35.09 & 20.88 & 2.52 & 2.76 & 27.65 \\
\hline $\mathbf{2 3}$ & 9.47 & 112.53 & 28.43 & 24.66 & 13.25 & 0.42 & 1.80 & 7.73 \\
\hline $\mathbf{2 4}$ & 0.78 & 109.91 & 24.45 & 20.43 & 11.82 & 0.35 & 2.13 & 9.94 \\
\hline
\end{tabular}

Note: $n=1461$ 
Table 3-3: Summary Statistics for PJM Day-Ahead Forward Price

\begin{tabular}{|c|c|c|c|c|c|c|c|}
\hline Hour & Min & Max & Mean & Median & $\begin{array}{c}\text { Standard } \\
\text { Deviation } \\
\sigma\end{array}$ & $\begin{array}{c}\text { Skewness } \\
\text { coefficient } \\
\mu_{3} / \sigma^{3}\end{array}$ & Kurtosis \\
\hline $\mathbf{1}$ & 5.00 & 81.68 & 22.79 & 20.27 & 9.56 & 2.33 & 10.82 \\
\hline $\mathbf{2}$ & 1.76 & 77.07 & 19.81 & 17.72 & 8.42 & 2.47 & 12.73 \\
\hline $\mathbf{3}$ & 0.68 & 72.45 & 18.34 & 16.58 & 8.17 & 2.46 & 13.37 \\
\hline $\mathbf{4}$ & 0.62 & 74.16 & 17.81 & 16.09 & 8.38 & 2.51 & 13.88 \\
\hline $\mathbf{5}$ & 0.18 & 80.04 & 18.77 & 16.62 & 9.17 & 2.55 & 13.32 \\
\hline $\mathbf{6}$ & 0.57 & 100.82 & 23.25 & 20.13 & 11.92 & 2.40 & 11.89 \\
\hline $\mathbf{7}$ & 0.91 & 154.69 & 33.01 & 27.42 & 19.65 & 1.82 & 8.03 \\
\hline $\mathbf{8}$ & 1.99 & 155.71 & 35.74 & 30.33 & 18.51 & 1.58 & 6.88 \\
\hline $\mathbf{9}$ & 11.01 & 153.00 & 36.84 & 33.83 & 15.79 & 1.57 & 7.92 \\
\hline $\mathbf{1 0}$ & 14.20 & 152.94 & 39.46 & 37.54 & 15.11 & 1.37 & 7.23 \\
\hline $\mathbf{1 1}$ & 15.07 & 177.68 & 41.90 & 40.24 & 16.35 & 1.80 & 11.56 \\
\hline $\mathbf{1 2}$ & 15.32 & 320.01 & 42.00 & 40.18 & 18.97 & 4.67 & 55.39 \\
\hline $\mathbf{1 3}$ & 15.12 & 384.11 & 41.35 & 38.75 & 22.05 & 6.45 & 82.91 \\
\hline $\mathbf{1 4}$ & 14.02 & 550.01 & 42.16 & 38.23 & 28.03 & 8.87 & 132.24 \\
\hline $\mathbf{1 5}$ & 13.93 & 700.00 & 41.85 & 35.92 & 33.98 & 10.63 & 171.04 \\
\hline $\mathbf{1 6}$ & 14.00 & 701.03 & 42.19 & 35.24 & 34.96 & 9.92 & 153.86 \\
\hline $\mathbf{1 7}$ & 15.04 & 650.01 & 44.90 & 39.38 & 32.75 & 9.08 & 136.68 \\
\hline $\mathbf{1 8}$ & 15.03 & 500.01 & 50.55 & 45.64 & 28.28 & 6.18 & 79.00 \\
\hline $\mathbf{1 9}$ & 14.91 & 450.01 & 49.07 & 45.01 & 24.37 & 4.88 & 64.21 \\
\hline $\mathbf{2 0}$ & 15.12 & 356.98 & 46.45 & 43.92 & 21.08 & 4.15 & 47.41 \\
\hline $\mathbf{2 1}$ & 17.09 & 296.58 & 45.37 & 43.80 & 18.56 & 3.01 & 30.98 \\
\hline $\mathbf{2 2}$ & 15.10 & 178.78 & 39.26 & 37.71 & 15.55 & 1.99 & 14.03 \\
\hline $\mathbf{2 3}$ & 13.87 & 106.70 & 30.09 & 28.26 & 11.11 & 1.60 & 7.87 \\
\hline $\mathbf{2 4}$ & 3.00 & 96.89 & 25.26 & 22.90 & 9.82 & 1.88 & 8.99 \\
\hline
\end{tabular}

Note: $n=1461$ 
Table 3-4: Summary Statistics for Forward Premium

\begin{tabular}{|c|c|c|c|c|c|c|c|}
\hline Hour & Min & Max & Mean & Median & $\begin{array}{c}\text { Standard } \\
\text { Deviation } \\
\sigma\end{array}$ & $\begin{array}{c}\text { Skewness } \\
\text { coefficient } \\
\mu_{3} / \sigma^{3}\end{array}$ & Kurtosis \\
\hline $\mathbf{1}$ & -31.89 & 47.49 & 0.56 & 0.33 & 6.77 & 1.22 & 9.81 \\
\hline $\mathbf{2}$ & -39.21 & 38.17 & -1.25 & -0.77 & 6.23 & -0.40 & 9.23 \\
\hline $\mathbf{3}$ & -30.38 & 36.84 & -0.82 & -0.46 & 5.76 & -0.01 & 8.94 \\
\hline $\mathbf{4}$ & -36.65 & 39.11 & -0.72 & -0.33 & 5.93 & 0.15 & 12.34 \\
\hline $\mathbf{5}$ & -38.23 & 42.91 & -1.05 & -0.71 & 6.29 & 0.29 & 11.08 \\
\hline $\mathbf{6}$ & -36.45 & 68.62 & -0.79 & -0.91 & 8.68 & 1.49 & 11.47 \\
\hline $\mathbf{7}$ & -60.60 & 72.02 & -0.27 & -0.66 & 13.84 & 0.50 & 5.69 \\
\hline $\mathbf{8}$ & -50.96 & 65.06 & -0.23 & 0.13 & 12.93 & 0.18 & 5.63 \\
\hline $\mathbf{9}$ & -37.90 & 64.89 & 0.84 & 0.85 & 11.35 & 0.58 & 5.59 \\
\hline $\mathbf{1 0}$ & -44.41 & 69.68 & 0.25 & -0.06 & 10.90 & 0.51 & 6.35 \\
\hline $\mathbf{1 1}$ & -74.79 & 113.38 & -1.69 & -1.64 & 12.71 & 0.94 & 16.54 \\
\hline $\mathbf{1 2}$ & -211.54 & 231.24 & -1.24 & -0.73 & 19.60 & -1.28 & 63.74 \\
\hline $\mathbf{1 3}$ & -333.67 & 253.69 & -1.58 & -0.74 & 25.96 & -4.67 & 89.43 \\
\hline $\mathbf{1 4}$ & -416.37 & 327.33 & -3.59 & -2.13 & 31.24 & -4.33 & 98.89 \\
\hline $\mathbf{1 5}$ & -405.12 & 412.66 & -0.50 & 0.02 & 32.11 & -1.36 & 101.92 \\
\hline $\mathbf{1 6}$ & -256.92 & 528.35 & 0.60 & 0.50 & 31.29 & 5.44 & 117.42 \\
\hline $\mathbf{1 7}$ & -307.94 & 424.34 & -0.75 & -0.17 & 30.67 & 1.43 & 77.76 \\
\hline $\mathbf{1 8}$ & -327.21 & 277.88 & 0.67 & 0.62 & 27.31 & -2.21 & 70.18 \\
\hline $\mathbf{1 9}$ & -159.41 & 348.57 & 3.89 & 2.61 & 20.95 & 3.54 & 76.25 \\
\hline $\mathbf{2 0}$ & -82.05 & 291.28 & 3.88 & 2.45 & 17.43 & 5.11 & 76.70 \\
\hline $\mathbf{2 1}$ & -221.36 & 170.14 & -0.79 & -0.55 & 18.44 & -4.42 & 67.73 \\
\hline $\mathbf{2 2}$ & -71.50 & 127.09 & -0.45 & -0.83 & 11.74 & 1.66 & 24.27 \\
\hline $\mathbf{2 3}$ & -34.71 & 68.74 & 1.64 & 0.94 & 8.38 & 1.29 & 9.84 \\
\hline $\mathbf{2 4}$ & -24.85 & 64.19 & 0.75 & 0.35 & 6.88 & 1.31 & 11.76 \\
\hline
\end{tabular}

Note: $n=1453$ 
Figure 3-1: Simulated Effect of Natural Gas Availability on Electricity Price Skewness

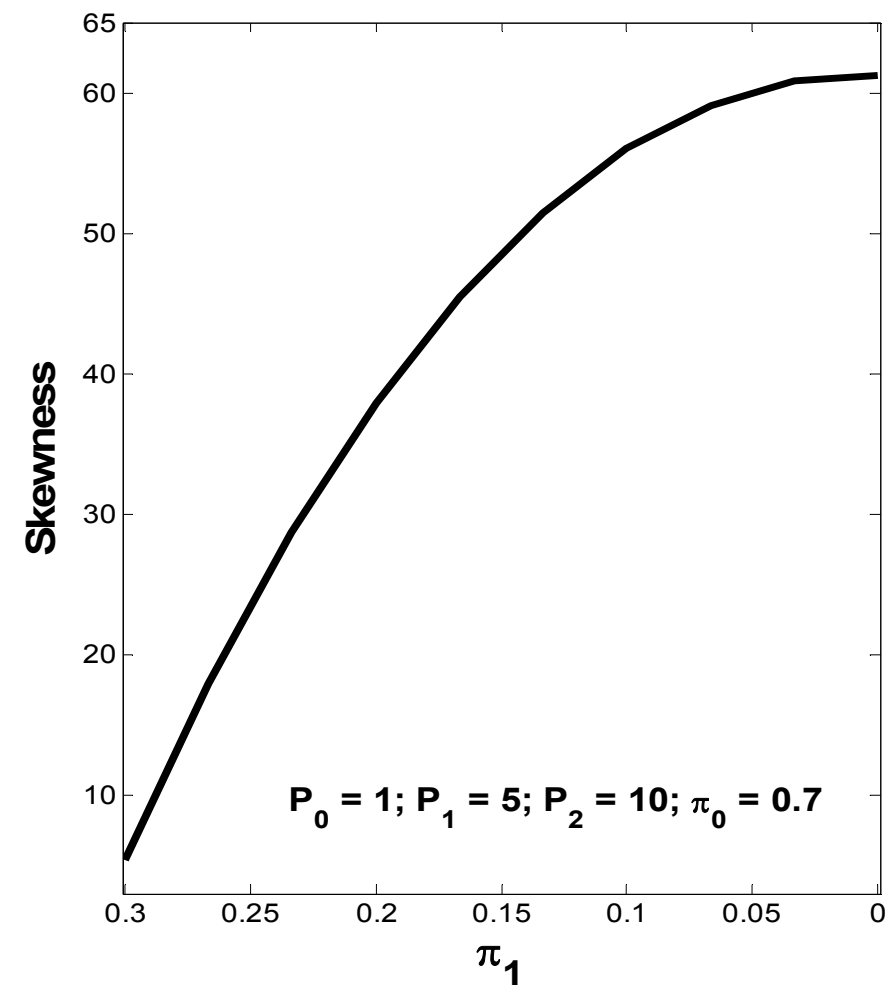


Figure 3-2: Estimated Effects of Recent Spot Price Skewness, and Variance on the Electricity Forward Premium, with $95 \%$ Confidence Intervals

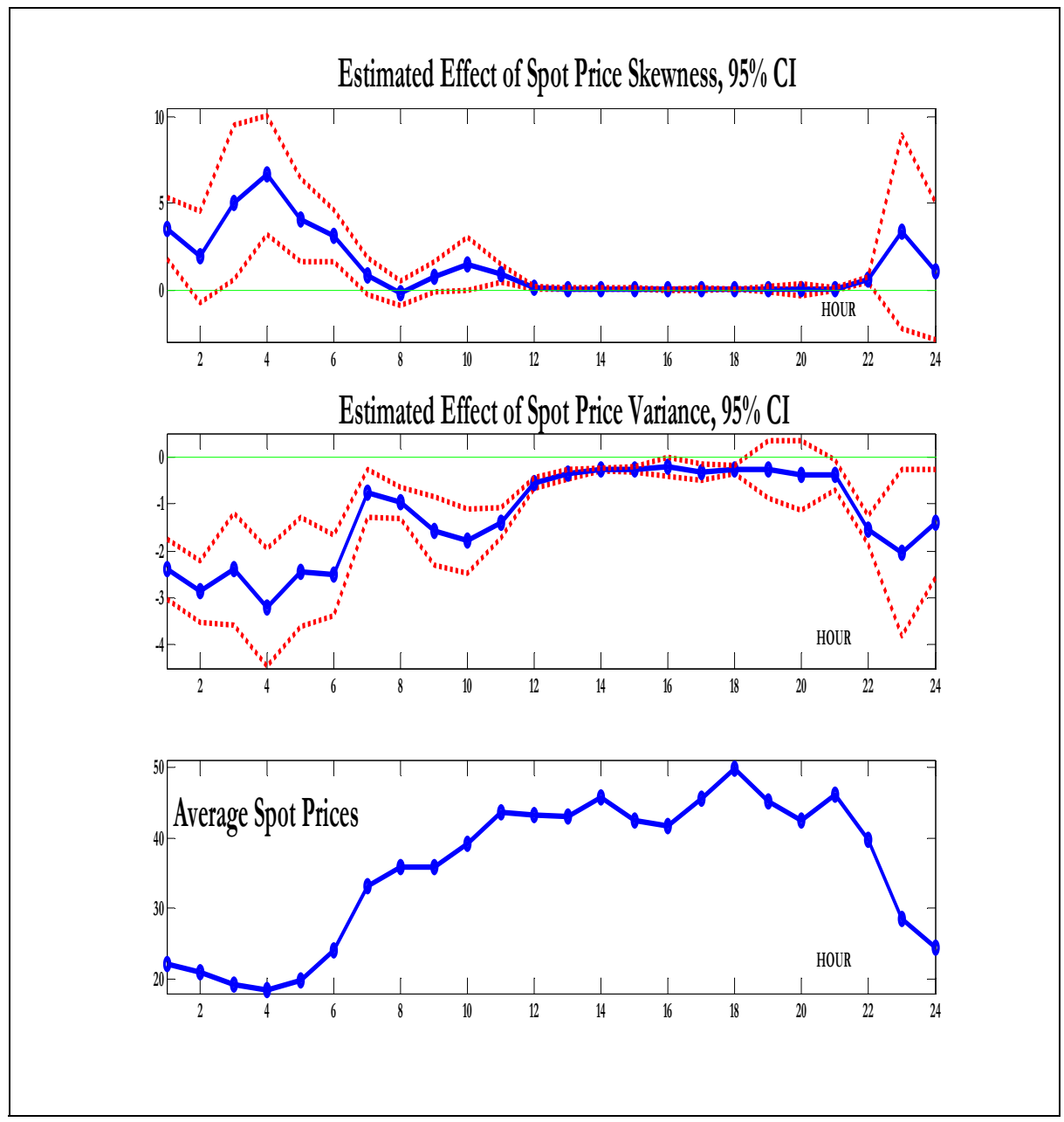



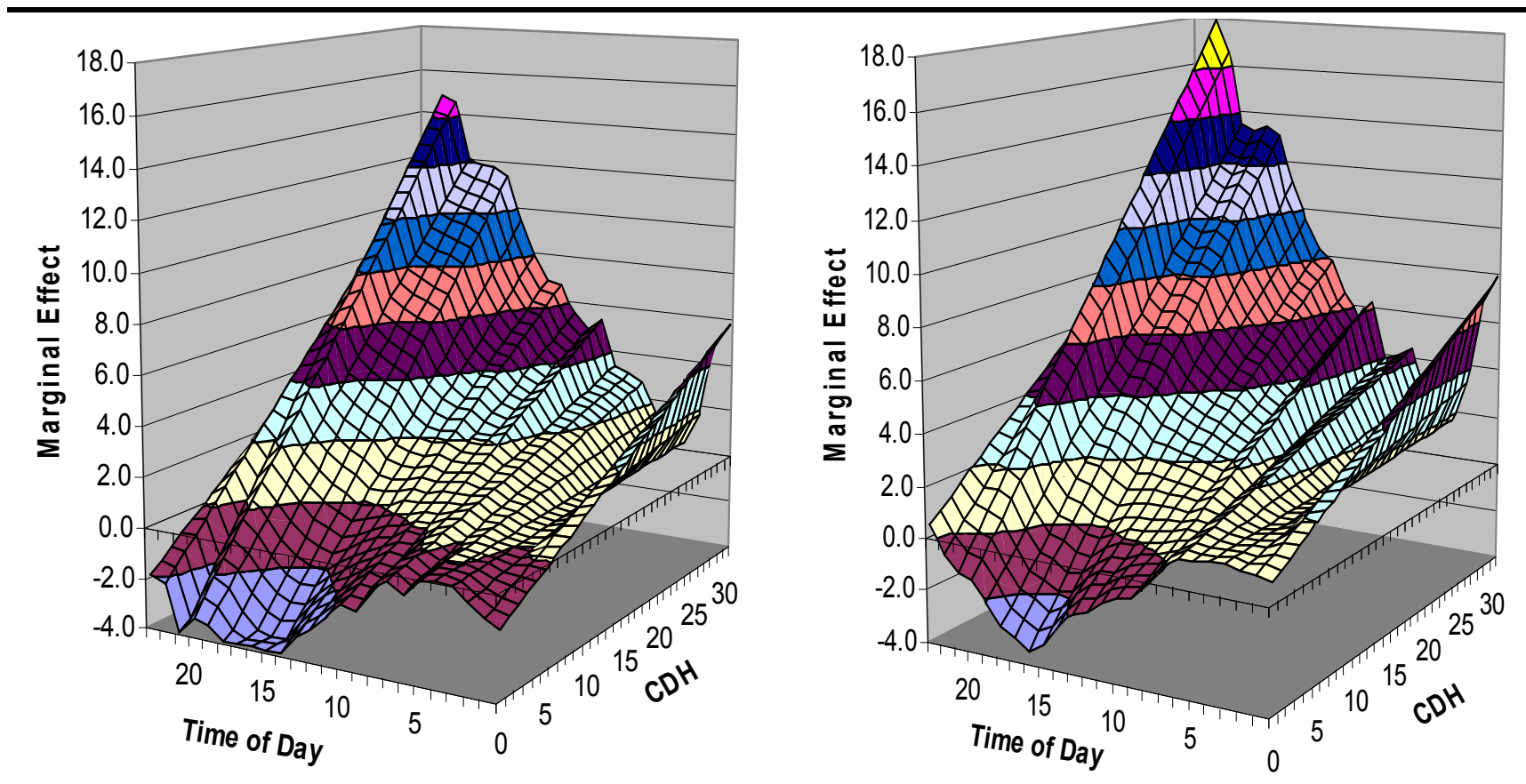

High Gas Storage Inventories (GS = 1900)

Low Gas Storage Inventories (GS $=600$ )

Figure 3-3: Estimated Marginal Effect of Temperature on the Forward Premium

$\left(\beta_{4 a}+2 \beta_{4 b} \mathrm{CDH} t+\beta_{6} \mathrm{GSt}-1\right)$, by Levels of Gas Storage, Temperature, and Time of Day

(Note: Temperature Fahrenheit $=\mathrm{CDH}+65$ ) 
Table 3-5: Regression Results. Dependent Variable: Forward Premium

Premium $_{\mathrm{t}}=\beta_{0}+\beta_{1} \operatorname{Var}_{\mathrm{t}-1}(\mathrm{~S})+\beta_{2} \operatorname{Skew}_{\mathrm{t}-1}(\mathrm{~S})+\beta_{3} \mathrm{GS} \mathrm{t-1}+\beta_{4} C D H_{\mathrm{t}}+\beta_{5} H D H_{\mathrm{t}}+\beta_{6} G S_{\mathrm{t}-1}{ }^{*} C D H_{\mathrm{t}}+\beta_{7} G S_{\mathrm{t}-1}{ }^{*} H D H_{\mathrm{t}}+\varepsilon_{\mathrm{t}}$

\begin{tabular}{|c|c|c|c|c|c|c|}
\hline & Midnite & $1 \mathrm{am}$ & 2am & 3am & 4am & 5 am \\
\hline \multirow{2}{*}{$\beta_{0}:$ Constant } & -0.62 & -1.54 & $-1.87^{* *}$ & $-1.57^{*}$ & -1.25 & $-1.86^{\star \star}$ \\
\hline & -0.61 & -1.6 & -2.44 & -1.86 & -1.59 & -2.1 \\
\hline \multirow{2}{*}{ 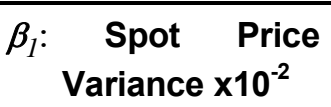 } & $-1.41^{* *}$ & $-2.40^{* * *}$ & $-2.87^{* * *}$ & $-2.40^{\star * *}$ & -3.21 & $-2.46^{* * *}$ \\
\hline & -2.42 & -7.45 & -8.49 & -3.95 & -5.04 & -4.14 \\
\hline \multirow{2}{*}{ 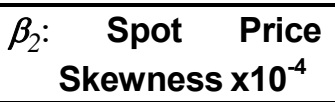 } & 1.06 & $3.54^{* \star \star}$ & 1.92 & $5.03^{\star \star}$ & $6.64^{\star \star \star}$ & $4.04^{\star \star \star}$ \\
\hline & 0.52 & 3.93 & 1.42 & 2.2 & 3.79 & 3.28 \\
\hline \multirow{2}{*}{$\begin{array}{c}\beta_{4 a}: \text { CDH (Cooling } \\
\text { Degree Hours) }\end{array}$} & $1.76^{\star \star \star}$ & $1.84^{* \star *}$ & $1.68^{* \star \star}$ & $1.72^{\star \star \star}$ & $1.57^{\star \star *}$ & $1.54^{* \star \star}$ \\
\hline & 3.32 & 3.5 & 3.28 & 3.73 & 3.16 & 3.55 \\
\hline \multirow{2}{*}{$\beta_{4 b}:$ CDH Squared } & $0.12^{\star \star \star}$ & $0.10^{* * *}$ & 0.04 & 0.01 & 0.01 & 0.03 \\
\hline & 4.58 & 3.8 & 1.47 & 0.52 & 0.42 & 0.92 \\
\hline \multirow{2}{*}{$\begin{array}{c}\beta_{5 a}: \text { HDH (Heating } \\
\text { Degree Hours) }\end{array}$} & -0.06 & $-0.19^{\star *}$ & $-0.13^{*}$ & -0.11 & $-0.16^{* *}$ & -0.08 \\
\hline & -0.7 & -2.01 & -1.82 & -1.58 & -2.18 & -1.05 \\
\hline \multirow{2}{*}{$\beta_{5 b}:$ HDH Squared } & $0.01^{* \star *}$ & $0.01^{* * *}$ & $0.01^{* * *}$ & $0.01^{* * *}$ & $0.01^{* * *}$ & $0.01^{* * *}$ \\
\hline & 5.03 & 7.33 & 6.47 & 5.27 & 6.24 & 4.77 \\
\hline \multirow{2}{*}{$\begin{array}{c}\beta_{3}: \underset{t-1}{\text { Storage } \times 10^{-3}} \text { Gas } \\
\end{array}$} & 0.49 & $1.77^{\star \star}$ & $1.20^{*}$ & 0.63 & 0.77 & 0.51 \\
\hline & 0.59 & 2.23 & 1.86 & 0.92 & 1.1 & 0.69 \\
\hline \multirow{2}{*}{$\beta_{6}: \mathrm{CDH} \times \mathbf{G S}_{t-1} \times 10^{-4}$} & $-16.5^{\star \star \star}$ & $-15.6^{* * *}$ & $-12.1^{* * *}$ & $-9.81^{* * *}$ & $-8.57^{* *}$ & $-9.03^{* * *}$ \\
\hline & -3.79 & -3.8 & -3.08 & -2.74 & -2.21 & -2.68 \\
\hline \multirow{2}{*}{$\beta_{7}: \mathrm{CDH} \mathbf{G S}_{t-1} \times 10^{-4}$} & $-1.05^{\star}$ & $-1.15^{\star *}$ & $-0.75^{\star}$ & -0.59 & -0.66 & $-0.85^{\star}$ \\
\hline & -1.7 & -2.1 & -1.85 & -1.41 & -1.34 & -1.81 \\
\hline$R^{2}$-adj & 0.25 & 0.34 & 0.39 & 0.27 & 0.27 & 0.24 \\
\hline
\end{tabular}

Note: t-statistics in parentheses. ${ }^{*}$ significant at $10 \%$ level; ${ }^{* *}$ significant at $5 \%$ level; *** significant at $1 \%$ level. 
Table 3-6: Regression Results. Dependent Variable: Forward Premium

Premium $_{\mathrm{t}}=\beta_{0}+\beta_{1} \operatorname{Var}_{\mathrm{t}-1}(\mathrm{~S})+\beta_{2} \operatorname{Skew}_{\mathrm{t}-1}(\mathrm{~S})+\beta_{3} \mathrm{GS} \mathrm{S}_{\mathrm{t}-1}+\beta_{4} C D H_{\mathrm{t}}+\beta_{5} H D H_{\mathrm{t}}+\beta_{6} \mathrm{GS} \mathrm{S}_{\mathrm{t}-1}{ }^{\star} C D H_{\mathrm{t}}+\beta_{7} G S_{\mathrm{t}-1}{ }^{*} H D H_{\mathrm{t}}+\varepsilon_{\mathrm{t}}$

\begin{tabular}{|c|c|c|c|c|c|c|}
\hline & 6am & $7 \mathrm{am}$ & 8am & 9am & $10 \mathrm{am}$ & $11 \mathrm{am}$ \\
\hline \multirow{2}{*}{$\beta_{0}:$ Constant } & -1.05 & 3.17 & 1.74 & $5.45^{\star * *}$ & $5.45^{\star \star \star}$ & 0.42 \\
\hline & -0.95 & 1.56 & 0.97 & 3.03 & 3.13 & 0.25 \\
\hline \multirow{2}{*}{$\beta_{1}: \begin{array}{c}\text { Spot } \\
\text { Variance } \\
\times 10^{-2}\end{array}$} & $-2.52^{\star * *}$ & $-0.77^{\star \star \star}$ & $-0.98^{\star \star \star}$ & $-1.58^{\star \star \star}$ & $-1.79 * * *$ & $-1.41^{* * *}$ \\
\hline & -5.83 & -2.94 & -5.89 & -4.27 & -5.15 & -8.54 \\
\hline \multirow{2}{*}{ 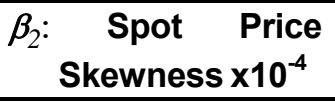 } & $3.10^{* \star \star}$ & 0.82 & -0.17 & $0.73^{*}$ & $1.50^{*}$ & $0.93^{* *}$ \\
\hline & 4.02 & 1.5 & -0.47 & 1.67 & 1.93 & 3.74 \\
\hline \multirow{2}{*}{$\begin{array}{c}\beta_{4 a}: \text { CDH (Cooling } \\
\text { Degree Hours) }\end{array}$} & $1.72^{\star \star \star}$ & $1.04^{*}$ & 0.02 & -0.38 & -0.56 & -0.73 \\
\hline & 3.24 & 1.85 & 0.05 & -0.91 & -1.27 & -1.1 \\
\hline \multirow{2}{*}{$\beta_{4 b}:$ CDH Squared } & 0.06 & $0.06^{*}$ & $0.07^{* * \star}$ & $0.12^{\star \star *}$ & $0.11^{* \star *}$ & 0.13 \\
\hline & 1.51 & 1.84 & 3.18 & 5.36 & 4.96 & 3.67 \\
\hline \multirow{2}{*}{$\begin{array}{c}\beta_{5 a}: \text { HDH (Heating } \\
\text { Degree Hours) }\end{array}$} & -0.03 & -0.06 & 0.11 & 0.07 & -0.03 & $-0.10^{*}$ \\
\hline & -0.31 & -0.49 & 0.84 & 0.49 & -0.22 & -0.67 \\
\hline \multirow{2}{*}{$\beta_{5 b}:$ HDH Squared } & $0.01^{* \star *}$ & $0.01^{\star \star *}$ & $0.01^{\star \star \star}$ & $0.01^{\star *}$ & $0.01^{* * *}$ & $0.01^{* * *}$ \\
\hline & 5.91 & 3.74 & 2.77 & 2.23 & 3.53 & 4 \\
\hline \multirow{2}{*}{$\begin{array}{c}\beta_{3}: \mathbf{G S}_{t-1} \text { Gas } \\
\text { Storage } \times 10^{-3}\end{array}$} & -0.21 & $-4.64^{\star \star \star}$ & -1.17 & $-2.79^{\star * *}$ & $-2.48^{\star \star}$ & 0.57 \\
\hline & -0.23 & -2.98 & -0.85 & -2.17 & -2.15 & 0.54 \\
\hline \multirow{2}{*}{$\beta_{6}: \mathrm{CDH} \times \mathrm{GS}_{t-1} \times 10^{-4}$} & $-13.2^{\star * *}$ & $-7.69^{*}$ & -4.14 & $-7.58^{\star \star *}$ & $-5.81^{* *}$ & $-7.99 * * *$ \\
\hline & -3.27 & -1.73 & -1.16 & -2.59 & -2.27 & -3.34 \\
\hline \multirow{2}{*}{$\beta_{7}: \mathrm{CDH} \times \mathbf{G S}_{t-1} \times 10^{-4}$} & -1.12 & -0.26 & -1.35 & -1.01 & -1.13 & $-1.32^{*}$ \\
\hline & -1.6 & -0.28 & 0.24 & -1.21 & -1.27 & -1.68 \\
\hline$R^{2}$-adj & 0.28 & 0.16 & 0.14 & 0.24 & 0.25 & 0.33 \\
\hline
\end{tabular}

Note: t-statistics in parentheses. ${ }^{*}$ significant at $10 \%$ level; ${ }^{* *}$ significant at $5 \%$ level;

${ }^{* * *}$ significant at $1 \%$ level. 
Table 3-7: Regression Results. Dependent Variable: Forward Premium

Premium $_{\mathrm{t}}=\beta_{0}+\beta_{1} \operatorname{Var}_{\mathrm{t}-1}(\mathrm{~S})+\beta_{2} \operatorname{Skew}_{t-1}(\mathrm{~S})+\beta_{3} \mathrm{GS} \mathrm{S}_{\mathrm{t}-1}+\beta_{4} C D H_{\mathrm{t}}+\beta_{5} H D H_{\mathrm{t}}+\beta_{6} G S_{\mathrm{t}-1}{ }^{*} C D H_{\mathrm{t}}+\beta_{7} G S_{\mathrm{t}-1}{ }^{*} H D H_{\mathrm{t}}+\varepsilon_{\mathrm{t}}$

\begin{tabular}{|c|c|c|c|c|c|c|}
\hline & Noon & $1 \mathrm{pm}$ & $2 \mathrm{pm}$ & $3 p m$ & $4 \mathrm{pm}$ & $5 \mathrm{pm}$ \\
\hline \multirow{2}{*}{$\beta_{0}:$ Constant } & -1.27 & -4.07 & $-7.21^{* *}$ & -0.09 & -2.46 & $-5.96^{*}$ \\
\hline & -0.59 & -1.47 & -2.27 & -0.03 & -0.60 & -1.69 \\
\hline \multirow{2}{*}{$\begin{array}{l}\beta_{1}: \text { Spot Price } \\
\text { Variance } \times 10^{-2}\end{array}$} & $-0.57^{* \star *}$ & $-0.37^{* * *}$ & $-0.27^{\star \star *}$ & $-0.26^{\star \star *}$ & $-0.21^{\star \star}$ & $-0.32^{* \star *}$ \\
\hline & -10.35 & -6.77 & -19.97 & -8.53 & -1.99 & -3.72 \\
\hline \multirow{2}{*}{$\begin{array}{l}\beta_{2}: \text { Spot Price } \\
\text { Skewness } \times 10^{-4}\end{array}$} & $0.12^{* * *}$ & $0.06^{\star * *}$ & $0.06^{* *}$ & $0.06^{* \star}$ & 0.02 & $0.06^{*}$ \\
\hline & 5.25 & 3.13 & 2.30 & 2.40 & 1.03 & 1.94 \\
\hline \multirow{2}{*}{$\begin{array}{l}\beta_{4 a}: \text { CDH (Cooling } \\
\text { Degree Hours) }\end{array}$} & -1.19 & -1.19 & -1.67 & $-2.46^{*}$ & -2.87 & -2.47 \\
\hline & -1.12 & -0.99 & -1.19 & -1.89 & -1.34 & -1.47 \\
\hline \multirow{2}{*}{$\beta_{4 b}:$ CDH Squared } & $0.16^{\star \star \star}$ & $0.17^{\star \star \star *}$ & $0.20^{\star \star \star}$ & $0.24^{* * *}$ & $0.28^{* *}$ & $0.28^{* * *}$ \\
\hline & 2.85 & 2.85 & 2.89 & 3.61 & 2.57 & 3.11 \\
\hline \multirow{2}{*}{$\begin{array}{ll}\beta_{5 a}: \quad \text { HDH } & \text { (Heating } \\
\text { Degree Hours) } & \\
\end{array}$} & -0.19 & -0.25 & -0.23 & $-0.37^{*}$ & -0.30 & -0.01 \\
\hline & -0.99 & -1.18 & -0.99 & -1.69 & -0.97 & -0.04 \\
\hline \multirow{2}{*}{$\beta_{5 b}:$ HDH Squared } & $0.01^{* * *}$ & $0.01^{* * *}$ & $0.01^{* \star \star}$ & $0.02^{\star \star \star}$ & $0.02^{\star \star \star}$ & $0.02^{* * *}$ \\
\hline & 3.03 & 3.36 & 2.72 & 4.21 & 3.30 & 4.04 \\
\hline \multirow{2}{*}{$\begin{array}{l}\beta_{3}: \mathbf{G S}_{t-1} \text { Gas Storage } \\
\mathbf{x} 10^{-3}\end{array}$} & 1.58 & $3.52^{* *}$ & $4.51^{* * *}$ & 1.82 & $4.19^{* *}$ & $5.48^{* * *}$ \\
\hline & 1.28 & 2.42 & 2.91 & 1.31 & 2.37 & 3.57 \\
\hline \multirow{2}{*}{$\beta_{6}: \mathbf{C D H} \times \mathrm{GS}_{t-1} \times 10^{-4}$} & $-8.75^{\star \star \star}$ & $-11.0^{* * *}$ & $-12.5^{\star * *}$ & $-13.0^{\star \star *}$ & $-12.9^{* * *}$ & $-13.4^{* * *}$ \\
\hline & -3.28 & -3.56 & -3.80 & -4.11 & -3.38 & -3.37 \\
\hline \multirow{2}{*}{$\beta_{7}: \mathrm{CDH} \times \mathrm{GS}_{t-1} \times 10^{-4}$} & -1.06 & -1.10 & -0.64 & -0.53 & -1.34 & $-3.40^{* \star *}$ \\
\hline & -1.09 & -1.03 & -0.75 & -0.68 & -1.34 & -3.60 \\
\hline$R^{2}$-adj & 0.57 & 0.67 & 0.61 & 0.49 & 0.37 & 0.47 \\
\hline
\end{tabular}

Note: t-statistics in parentheses. ${ }^{*}$ significant at $10 \%$ level; ${ }^{* *}$ significant at $5 \%$ level;

${ }^{* * *}$ significant at $1 \%$ level. 
Table 3-8: Regression Results. Dependent Variable: Forward Premium

Premium $_{\mathrm{t}}=\beta_{0}+\beta_{1} \operatorname{Var}_{\mathrm{t}-1}(\mathrm{~S})+\beta_{2} \mathrm{Skew}_{\mathrm{t}-1}(\mathrm{~S})+\beta_{3} \mathrm{GS} \mathrm{t}-1+\beta_{4} C D H_{\mathrm{t}}+\beta_{5} H D H_{\mathrm{t}}+\beta_{6} \mathrm{GS}_{\mathrm{t}-1}{ }^{*} C D H_{\mathrm{t}}+\beta_{7} \mathrm{GS} \mathrm{S}_{\mathrm{t}-1}{ }^{*} H D H_{\mathrm{t}}+\varepsilon_{\mathrm{t}}$

\begin{tabular}{|c|c|c|c|c|c|c|}
\hline & $6 \mathrm{pm}$ & $7 \mathrm{pm}$ & 8pm & 9pm & $10 \mathrm{pm}$ & $11 \mathrm{pm}$ \\
\hline \multirow{2}{*}{$\beta_{0}$ : Constant } & 0.10 & 3.74 & $5.30^{\star * *}$ & -1.39 & 1.69 & 0.73 \\
\hline & 0.04 & 1.44 & 2.73 & -0.72 & 1.10 & 0.50 \\
\hline Spot & $-0.26^{\star \star \star}$ & -0.27 & -0.39 & $-0.39^{* *}$ & $-1.55^{* \star *}$ & $-2.04^{\star \star}$ \\
\hline Variance $\times 10^{-2}$ & -6.11 & -0.88 & -1.04 & -2.29 & -10.00 & -2.27 \\
\hline \multirow{2}{*}{ 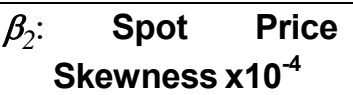 } & $0.04^{* *}$ & 0.02 & 0.00 & 0.04 & $0.56^{* * *}$ & 3.33 \\
\hline & 2.32 & 0.24 & 0.01 & 1.17 & 6.84 & 1.17 \\
\hline \multirow{2}{*}{ 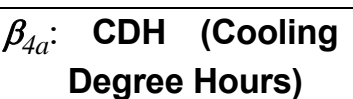 } & -2.31 & -1.46 & -0.45 & 0.23 & 0.18 & $1.56^{* * *}$ \\
\hline & -1.58 & -0.94 & -0.29 & 0.20 & 0.18 & 2.70 \\
\hline \multirow{2}{*}{$\beta_{4 b}:$ CDH Squared } & $0.27^{* \star *}$ & $0.26^{\star \star \star}$ & $0.30^{\star *}$ & $0.32^{\star *}$ & $0.24^{* *}$ & $0.18^{\star \star \star}$ \\
\hline & 3.21 & 2.77 & 2.42 & 3.04 & 2.52 & 3.81 \\
\hline \multirow{2}{*}{$\begin{array}{c}\beta_{5 a}: \text { HDH (Heating } \\
\text { Degree Hours) }\end{array}$} & 0.04 & $-0.66^{\star *}$ & -0.09 & 0.00 & -0.20 & -0.06 \\
\hline & 0.16 & -2.43 & -0.37 & 0.02 & -1.48 & -0.50 \\
\hline \multirow{2}{*}{$\beta_{5 b}:$ HDH Squared } & $0.02^{\star * *}$ & $0.02^{\star \star \star *}$ & $0.01^{* * *}$ & $0.01^{* * *}$ & $0.01^{* * *}$ & $0.01^{* \star *}$ \\
\hline & 3.58 & 4.42 & 2.90 & 3.50 & 3.56 & 3.46 \\
\hline \multirow{2}{*}{$\begin{array}{c}\beta_{3}: \underset{t-1}{\mathbf{G S}_{t-1}}{ }^{\text {Storagex } 10^{-3}} \\
\end{array}$} & 2.09 & -0.52 & $-4.11^{* *}$ & 1.14 & 0.45 & 0.88 \\
\hline & 1.28 & -0.27 & -2.45 & 0.76 & 0.37 & 0.83 \\
\hline \multirow{2}{*}{$\beta_{6}: \mathrm{CDH} \times \mathrm{GS}_{t-1} \times 10^{-4}$} & $-10.6^{* *}$ & $-10.7^{* *}$ & $-15.2^{\star \star \star}$ & $-24.0^{\star \star \star *}$ & $-12.7^{\star \star \star}$ & $-18.3^{\star \star \star}$ \\
\hline & -2.57 & -2.08 & -3.20 & -4.75 & -2.66 & -4.31 \\
\hline \multirow{2}{*}{$\beta_{7}: \mathrm{CDH} \times \mathrm{GS}_{t-1} \times 10^{-4}$} & $-4.00^{\star \star \star *}$ & 0.88 & -0.20 & $-1.89^{\star *}$ & -1.05 & -1.10 \\
\hline & -3.35 & 0.83 & -0.19 & -2.15 & -1.42 & -1.55 \\
\hline$R^{2}$-adj & 0.51 & 0.40 & 0.41 & 0.62 & 0.35 & 0.27 \\
\hline
\end{tabular}

Note: t-statistics in parentheses. ${ }^{*}$ significant at $10 \%$ level; ${ }^{* *}$ significant at $5 \%$ level;

${ }^{* * *}$ significant at $1 \%$ level. 


\section{Chapter 4 : Do Electricity Prices Follow Random Walk}

\section{Introduction}

Over the past two decades deregulation of electricity markets has taken place over the world. In general, deregulation of the electricity industry has aimed to increase overall market and economic efficiency: (1) to enhance competition (increase productive efficiency); and (2) to attract new investment (improve asset allocation efficiency). In economic literature, markets are called informational efficient if the market prices can fully reflect available and market-relevant information, so market agents can not gain abnormal profits on regular basis by devising a risk-free strategy. In this paper we attempt to study informational efficiency of the PJM electricity markets by testing for random walks in the electricity prices set in the real-time and day-ahead markets.

For this study hourly prices of day-ahead and real-time PJM markets are used, covering period of 01 June 2000 - 31 May 2006. Portmanteau white noise, unit root, and random walk multivariate variance ratio tests are used to examine the level of informational efficiency. This research finds that different tests lead to opposite conclusions on the informational efficiency of the market. In this light, major drawbacks and deficiencies of these tests are discussed.

The basic result indicates that economic efficiency, appropriately measured, is not a key characteristic of the PJM electricity markets. The null hypothesis of zero price return autocorrelation can be rejected. On one hand, the null hypothesis of unit root cannot be rejected for some set of hours in both markets signaling that there is some degree of efficiency. On the other hand, the null hypothesis of a random walk can be rejected for morning and night hours, while there is evidence that afternoon markets for electricity are informational efficient.

The significance of this research is threefold. First, we offer empirical testing for market efficiency. Once informational efficiency is rejected, sufficient prediction of electricity prices can be 
achieved by employing stochastic autoregressive modeling techniques. Second, comparison of different econometric tests results is offered. The differences in the results are discussed. Third, improving our understanding of the informational efficiency of the electricity markets will improve our understanding of the market designs and well as consequences of deregulation.

The paper is organized into 6 sections. Following this introduction, section 2 provides a brief description of market efficiency theory. Section 3 summarizes previous studies done on testing electricity markets efficiency and methodology. Section 4 describes the PJM markets and data used in the work. Section 5 reports our empirical results and offers discussion of them. Section 6 concludes, and suggests some avenues for future research.

\section{Market Efficiency: Theory}

Roberts (1967) described three forms of the Efficient Market Hypothesis (EMH) - weak, semi-strong, and strong - distinguished by different types of available information. The weak form of EMH assumes that market prices fully reflect the information contained in the historical observations, so it is not possible to construct a trading strategy to gain abnormal profits analyzing the past price paths. According to Samuelson (1965), in a weak-form efficient market the price dynamics fully reflects the influx of new information. If news arrives randomly then prices should follow a random walk meaning that the weak-form EMH is equivalent to the "random walk hypothesis." The semi-strong form of the EMH asserts that current prices reflect not only historical information but also all publicly available information relevant to the market. If a market is efficient in this sense, then an analysis of any public information (including but not limited to companies' balance sheets, income statements, weather conditions, and prices of fuels) will not help to earn abnormal profits repeatedly. The strong form of EMH states that all information - public and private - known to any market participant about market conditions is fully reflected in price dynamics. Therefore, it is possible to reveal private information from current market prices. In this paper we 
study the weak form of the Efficient Market Hypothesis (or Random Walk Hypothesis), referring to the prices predictability based on the information gleaned from the past ${ }^{4}$

Economic theory and empirical evidence of informational efficiency of the electricity markets is very important, because there is no possibility of profitable speculation in efficient markets that are in equilibrium. If prices follow random walk, the current price, $X_{t}$, can be viewed as an initial value, $X_{0}$, plus the cumulative sum of all random news - called shocks or innovations arrived since the beginning of the process, $\sum_{j=1}^{t} \varepsilon_{j}$. This implies that the impact of news does not die away and if news shocks are independent then the best predictor of the future price is its present realization.

As with the $\mathrm{EMH}$ there are three different versions of the random walk hypothesis, described by Campbell et al (1997). The strongest version assumes independent and identically distributed (i.i.d.) shocks ${ }^{5}$. It says that there is absolutely no information on change in price from previous to current value (e.g. on price return/change/increment) that can be revealed from the past, meaning that only currently available information is relevant for predicting the next-period price. The second version assumes that price shocks are only independent, meaning that innovations can come from different but independent distributions. This makes the second version of the random walk the most challenging to test. The third, and the least stringent version of the random walk hypothesis, assumes that price innovations are uncorrelated. Absence of correlation in price returns makes it is difficult to predict ${ }^{6}$ future prices using observed historical dynamics ${ }^{7}$.

${ }^{4}$ Fama $(1970,1991)$ offers a good review of empirical studies on each of the EMH forms.

${ }^{5}$ In the case of random walk, price changes/returns are equivalent to price shocks/innovations. $X_{t^{-}}$ $\mathrm{X}_{\mathrm{t}-1}=\mathrm{X}_{\tilde{0}}+\varepsilon_{\mathrm{t}}$, where $\mathrm{X}_{\tilde{0}}$ is a fixed level.

${ }^{6}$ At least to predict using information carried in the first moments, while some prediction power can be concentrated in the second and even third moments of distribution. In this case GARCH-types models may be useful for modeling.

${ }^{7}$ Normally distributed and uncorrelated price shocks are independent. 
In the econometric literature there are several tests developed for the third version of random walk detection, including Portmanteau or Q tests, unit root tests (also called stationary tests), and variance ratio test. These tests are discussed in details below.

\section{Market Efficiency: Literature and Tests}

The question of market efficiency in electricity markets has been raised in previous empirical literature that focused on the electricity prices. De Vany and Walls (1999a, 1999b) analyze the price dynamics of 11 interconnected regional markets in the western USA. Utilizing cointegration analysis for evidence of market integration, the authors found that the markets they consider were efficient, integrated, and stable. Von der Fehr and Harbord (1998) compare the level of competition - measures of which they base on the predictions from auction theory - between the electricity markets of Australia, England, Norway, and Wales, finding that the differences in the degree of competition level across these electricity markets can be successfully explained by the differences in market designs. Wolfram (1999) empirically analyzes economic efficiency in the electricity market, by examining generators cost-price mark-ups in the deregulated English power market during 1990s, using different measures of marginal costs ${ }^{8}$. The key finding is that prices charged by generators are significantly higher than marginal costs but lower than prices predicted by classical oligopoly theory. Li and Flynn (2004) compared diurnal patterns of 14 electricity markets located around the world, finding different degrees of predictability in prices and commenting on how this can affect customers' behavior and desire to hedge.

Borenstein et al. (2001) evaluates the level of integration between real-time and day-ahead markets in California, concluding that the price convergence between these two markets improved as markets become more mature. Expanding this finding, Arciniegas et al. (2003) evaluate the efficiency of the California, PJM, and New York markets. The authors offer a measure of market efficiency for each market for each of the years analyzed based on co-integration analysis of day-

\footnotetext{
${ }^{8}$ Marginal costs are believed to be a direct measure of market power
} 
ahead and real-time hourly price series, concluding that not only market efficiency has improved over time for all three power markets but also they have about the same level of efficiency.

Higgs and Worthington (2003) study the Australian electricity market efficiency by examining whether real-time prices follow a random walk utilizing multiple variance ratio test and concluding that this market is informational inefficient. Worthington and Higgs (2004) study the relationship between prices of the Australian spot and futures electricity markets. Findings the prices stationary, the authors apply GARCH technique to examine the markets convergence. They conclude that there is a significant informational spillover between the markets. Lu and Dong (2005) study the informational efficiency of the spot and futures Australian electricity markets by employing rational expectations model concluding the markets are efficient. Feng et al (2007) study Nordic electricity market, by employing vector error correction model, the authors find that the futures are unbiased predictors of real-time prices, and conclude that the Nordic futures market is an important tool in price discovery.

Emery and Liu (2002) study the relationship between electricity and natural gas futures traded on NYMEX. The authors found that futures prices for electricity and natural gas were cointegrated over 1996-2000. Shawky et al (2003) investigate the statistical properties of NYMEXtraded spot and futures electricity prices and their dynamic relationship. The authors also estimate risk premium and variance hedge ratios for the futures.

\section{III.1 Portmanteau (Ljung-Box Q) Test}

One way to test random walk hypothesis is to test whether or not the price returns show robust serial autocorrelation. In rigorous terms, the null hypothesis of no correlation is $H_{0}: \rho_{K}=0$, for all $\mathrm{K}$, where $\rho_{K}$ is the Kth order autocorrelation of price returns series. If the null can be rejected at least for some $\mathrm{K}$ then the price path is predictable in part by observing past behavior. A Portmanteau test is a test of the hypothesis that the first $\mathrm{K}$ autocorrelations are all not different from zeros statistically. 
The classical Portmanteau test statistic proposed by Box and Pierce (1970) has been shown to perform poorly in finite samples (i.e. its finite sample distribution is different from the asymptotic). Ljung and Box (1978) proposed a modified test statistic - widely used - called the Qstatistic. For the time series $X_{t}, t=0, \ldots, T$ the $\mathrm{Q}$-statistic for testing uncorrelatedness of price returns is given as:

$$
\begin{aligned}
& Q(k)=T(T+2) \sum_{t=1}^{k} \frac{\hat{\rho}^{2}(k)}{T-k} \\
& \hat{\rho}(k)=\hat{\gamma}(k) / \hat{\gamma}(0) \\
& \hat{\gamma}(k)=T^{-1} \sum_{t=1}^{T-k}\left(\Delta X_{t}-\overline{\Delta X}\right)\left(\Delta X_{t+k}-\overline{\Delta X}\right)
\end{aligned}
$$

Where $\mathrm{T}$ is the number of observations, $\Delta \mathrm{X}_{\mathrm{t}}=\mathrm{X}_{\mathrm{t}}-\mathrm{X}_{\mathrm{t}-1}$, and $\overline{\Delta X}$ is a sample mean of price changes, $\Delta X_{t}$. Under the null the test statistic $Q(K)$ - computed from the first $K$ sample autocorrelations - has a chi-square distribution with $\mathrm{K}$ degrees of freedom.

The Portmanteau test is powerful; however, if the null of no autocorrelation is rejected, separate autocorrelations have to be analyzed in order to see which one is causing the problem. In addition, the test seems to be sensitive to the choice of $\mathrm{K}$, and the larger the $\mathrm{K}$ the higher the likelihood of rejecting the null. Although strictly speaking, the Portmanteau test tests if correlations are zeros, this test applied to the price return series, $\Delta \mathrm{X}_{\mathrm{t}}$, is equivalent to testing whether the original time series, $X_{t}$, is a random walk.

\section{III.2 Unit Root Tests}

Since a random walk is a process with a unit root, unit root tests can be used for testing random walk hypothesis. Unfortunately, unit root tests are designed to check whether a time series have unit root, and do not look at predictability power of disturbances, which may have an important effect on price dynamics. In contrast, a pure test for random walk would not only test for a unit root but also examine the behavior of innovations. 
Numerous tests have been developed in the past to test for a unit root including the DickeyFuller (ADF) test (Fuller (1976), Dickey and Fuller (1979)), Dickey-Fuller Generalized Least Squares (DF-GLS) test (Elliot, Rosenberg, and Stock (1996)), Phillips-Perron test (Phillips (1987), Phillips and Perron (1988)) and Kwiatkowski-Phillips-Schmidt-Shin (KPSS) test (Kwiatkowski et al. (1992)). Since ADF and PP tests are asymptotically equivalent, we will focus on the ADF test solely.

\section{III.2.1 Augmented Dickey-Fuller Test}

Formal tests to determine whether a time series contains a stochastic trend are called unit root tests. The classical is the augmented Dickey-Fuller (ADF) test. Having a process $X_{t}, t=1, \ldots, T$, the ADF test is to find if $X_{t}$ has unit root or stationary (around a level or deterministic trend) by running the following test regressions:

$$
\begin{gathered}
\Delta \mathrm{X}_{\mathrm{t}}=\alpha_{0}+\alpha_{1} \mathrm{t}+\gamma \mathrm{X}_{\mathrm{t}}+\sum_{j=1}^{p} \beta_{j} \Delta X_{t-j}+\varepsilon_{t} \text { Process with a level and deterministic trend } \\
\Delta \mathrm{X}_{\mathrm{t}}=\alpha_{0}+\gamma \mathrm{X}_{\mathrm{t}}+\sum_{j=1}^{p} \beta_{j} \Delta X_{t-j}+\varepsilon_{t} \text { Process with a level }
\end{gathered}
$$

Here, $\Delta X_{t}=X_{t}-X_{t-1}$ is a time series return. The null hypothesis is whether the process is integrated: $H_{0}: \gamma=0$ (i.e. unit root present) and the alternative is $H_{1}: \gamma<0$ (i.e. time series is stationary). If the null hypothesis is rejected for the process with deterministic trend, it is worth testing the null of the unit root with a level only. The $p$ lagged regressors are included to reduce possible nuisance caused by the temporal dependencies in the disturbances, $\varepsilon_{t}$. It is well-know (see Koehler and Murphree (1988), Hall (1994), Ng and Peron (1995)) that ADF tests are sensitive to the choice of the number of lags, p. The classical selection criteria for $p$ are Akaike (1976) and Schwartz (1978). The optimal lag length for this work is selected based on the Modified Akaike Information Criterion, offered by $\mathrm{Ng}$ and Perron (2001). In addition to sensitivity of lag length, the ADF tests have low power against stationary alternative. Simply saying, the ADF test cannot discriminate highly persistent stationary process against unit root process (see Campbell and Perron (1991)). To deal 
with this Elliot et al (1996) offer an improvement to the ADF procedure, described in the next subsection.

\section{III.2.2 Dickey-Fuller Generalized Least Squares (DF-GLS) test}

The Dickey-Fuller test with GLS de-trending is a modification to the ADF test and it has been proposed by Elliot, Rothenberg and Stock (1996, hereafter ERS) as a more powerful alternative to the classical ADF test. The test "has substantially improved power when an unknown mean or trend is present" (ERS, p.813). Unlike the ADF test, where either a drift or a drift and a time trend is included into the test regression to capture the deterministic effects, the DF-GLS test considers time series that are "cleaned out" before the unit root test is performed. De-trending is done by taking the drift and/or and deterministic linear trend out of the data employing Generalized Least Squares technique. Therefore, the unit root test is conducted using the following test equation:

$$
\Delta \mathrm{X}_{\mathrm{t}}^{\mathrm{D}}=\gamma \mathrm{X}_{\mathrm{t}}^{\mathrm{D}}+\sum_{j=1}^{p} \beta_{j} \Delta X_{\mathrm{t}-\mathrm{j}}^{\mathrm{D}}+\varepsilon_{t}
$$

where $\Delta \mathrm{X}_{\mathrm{t}}^{\mathrm{D}}$ is the increment of the de-trended value of the examined variable $\mathrm{X}_{\mathrm{t}}$. As in the case of the ADF test, the null hypothesis of a unit root is $\mathrm{H}_{0}: \gamma=0$ and the alternative is $\mathrm{H}_{1}: \gamma<0$. The critical values can be found in ERS (Table 1), where critical values for the DF-GLS are approximately equal to those for the no-level and no-trend augmented Dickey-Fuller test.

\section{III.2.3 Stationarity Test (KPSS)}

The KPSS test (Kwiatkowski et al (1992)) is used to check whether an observable time series is stationary around a deterministic level or trend against the alternative of unit root. This test is well-known to be the most powerful. The KPSS test model for the examined variable, $X_{t}$, is $X_{t}=\alpha_{0}+\alpha_{1} t+r_{t}+\varepsilon_{t}$. In this model, $\alpha_{0}+\alpha_{1} t$ is a deterministic drift and time trend component, $r_{t}=r_{t-1}+u_{t}$ is a random walk process, and $t$ is a stationary disturbance term. The 
null hypothesis of stationary process implies that the random walk has zero variance $\mathrm{H}_{0}: \sigma_{u}^{2}=0$ (meaning that process $r_{t}$ is constant). The trend stationarity test's regression is $X_{t}=\alpha_{0}+\alpha_{1} t+e_{t}$; while under the level stationarity test's equation is $X_{t}=\alpha_{0}+e_{t}$. The partial sum of residuals $S_{t}=\sum_{i=1}^{t} \hat{e}_{i}-$ is used to obtain the test statistics $-K P S S=T^{-2} \sum_{t=1}^{T} S_{t}^{2} / \sigma^{2}$, where under the null hypothesis $\sigma^{2}$ is a consistent estimator of the long run variance of $\varepsilon_{t}\left(\sigma_{\varepsilon}^{2}\right)$ which is defined as $\sigma^{2}=\lim _{T \rightarrow \infty} T^{-1} \mathrm{E}\left(\left[\sum_{t=1}^{T} \varepsilon_{t}\right]^{2}\right)$. Den Haan and Levin (1997), suggest that the accuracy of KPSS test inference depends on the actual choice of estimator for $\sigma_{\varepsilon}^{2}$, discussion of which is beyond the scope of this work.

The KPSS test is most useful as a combination with other tests. For example, if using the ADF test one cannot reject the unit root null and using the KPSS test one can reject the stationarity null, then the data is integrated i.e. has unit root. On the other hand if using ADF one can reject the unit root null, and using KPSS can reject the stationarity null, then further examination is needed since tests contradict each other.

\section{III.3 Multiple Variance Ratio Test for Random Walk}

Lo and MacKinlay (1988, hereafter LMK) offered a simple variance ratio (VR) method for testing the random walk hypothesis against stationary alternatives. The test is based on assumption that the random walk increments are uncorrelated and their variance is linear in any and all sampling intervals. If the underlying process of time series is a random walk (with or without a level) then the variance of $n$-period increment is linearly proportional to the variance of one-period increment, where the coefficient of proportionality is exactly equal to $n(n>1)$. LMK have proved that this property holds asymptotically even for heteroscedastic disturbance terms. To restate in mathematical terms consider the random walk, $\left\{X_{0}, X_{1}, \ldots, X_{T n}\right\}$, with a level $X_{0}: X_{t}=X_{0}+X_{t-1}+\varepsilon_{t}$. 
Assuming that the disturbance term, $\varepsilon_{t}$, has a zero mean $E\left(\varepsilon_{t}\right)=0$, and zero correlation $E\left(\varepsilon_{t}, \varepsilon_{t n}\right)=0$, for all $n \neq 0$. Under the random walk hypothesis the $n$-period increments or returns, $\Delta_{n} X_{t}=X_{t}-X_{t-n}=n X_{0}$ $+\varepsilon_{t}+\varepsilon_{t-1}+\ldots+\varepsilon_{t-n+1}$, are uncorrelated and their variance is linear in $n$, for all $n>0$. In other words, for any random walk process the variance of $n$-period return, $\Delta_{n} X_{t}$, should be $n$ times larger than the variance of one-period return, $\Delta \mathrm{X}_{\mathrm{t}}=\mathrm{X}_{\mathrm{t}}-\mathrm{X}_{\mathrm{t}-1}: \sigma^{2}(\mathrm{n})=\operatorname{Var}\left(\mathrm{X}_{\mathrm{t}}-\mathrm{X}_{\mathrm{t}-\mathrm{n}}\right)=\operatorname{Var}\left(\Delta \mathrm{X}_{\mathrm{t}}+\Delta \mathrm{X}_{\mathrm{t}-1}+\ldots+\Delta \mathrm{X}_{\mathrm{t}-\mathrm{n}+1}\right)=$ $n \operatorname{Var}\left(\Delta \mathrm{X}_{\mathrm{t}}\right)=\mathrm{n} \sigma^{2}(1)$

For a process of $T n+1$ observations the consistent estimate of the variances is given as (see LMK p.47):

$$
\begin{aligned}
& \hat{\sigma}^{2}(n)=\kappa^{-1} \sum_{l=n}^{T n}\left(X_{l}-X_{l-n}-n \bar{X}\right)^{2} \\
& \hat{\sigma}^{2}(1)=(T n-1)^{-1} \sum_{l=1}^{T n}\left(X_{l}-X_{l-1}-\bar{X}\right)^{2}
\end{aligned}
$$

Where $\kappa=n(T n-n+1)(1-n / T n) ;$ and $\bar{X}=\sum_{l=1}^{T n+1}\left(X_{l}-X_{l-1}\right) / T n$ is the sample mean

of one-period increments. As it is shown in LMK (theorem 2 and 3), under the null hypothesis of random walk, both test statistics for homogeneous increments, $\hat{Z}_{0}(n)=(\hat{V}(n)-1) / \sigma_{0}(n)$, and for heteroscedastic increments, $\hat{Z}_{*}(n)=(\hat{V}(n)-1) / \sigma_{*}(n)$, have asymptotic standard normal distribution, where $\hat{V}(n)=\hat{\sigma}(n) / \hat{\sigma}(1)$ is so-called variance ratio,

$$
\begin{gathered}
\sigma_{0}^{2}(n)=2(2 n-1)(n-1) / 3 \operatorname{Tn}^{2} \quad \sigma_{*}^{2}(n)=4 \sum_{k=1}^{n-1}(1-k / n)^{2} \delta(k) \\
\delta(k)=\frac{\sum_{l=k+1}^{T n}\left(X_{l}-X_{l-1}-\bar{X}\right)^{2}\left(X_{l-k}-X_{l-k-1}-\bar{X}\right)^{2}}{\left(\sum_{l=1}^{T n}\left(X_{l}-X_{l-1}-\bar{X}\right)^{2}\right)^{2}}
\end{gathered}
$$


LMK procedure is appropriate for testing variance ratios for a specific value of $n$, however, while the random walk hypothesis requires that the variance ratios equal to unity for all $\mathrm{n}$. Chow and Denning (1993) design the multiple variance ratios (MVR) test procedure for testing the random walk hypothesis by comparing all variance ratios with unity.

For Multiple Variance Ratio test it is necessary to consider a set of subhypotheses $\left\{H_{0 i}: \hat{Z}\left(n_{i}\right)=0 \mid n_{1}=2<n_{2}<\ldots<n_{m} \leq \operatorname{Tn} / 2\right\}$ based on homogeneous or heteroscedastic random walk test statistics $\hat{Z}\left(n_{i}\right)$. Rejection of any of $\mathrm{H}_{0 \mathrm{i}}$ leads to rejection of the null of random walk. Chow and Denning (p.390) show that $\operatorname{Prob}\left(\hat{Z}^{+}(n) \leq Z_{\alpha^{+}}\right) \geq(1-\alpha)$ where $\hat{Z}^{+}(n)=\max _{1 \leq i \leq m}\left\{\left|\hat{Z}\left(n_{i}\right)\right|\right\}$ is the largest absolute value of the test statistic, $2 \alpha^{+}=1-(1-\alpha)^{m}$, and $Z_{\alpha^{+}}$is the upper $\alpha^{+}$point of the standard normal distribution.

Summing up, to test the null hypothesis of a random walk, one needs to calculate the values of the standardized test statistics, $\hat{Z}_{0}(n)$ and $\hat{Z}_{*}(n)$, for all values of $n \geq 2$, choose the one with the largest absolute value and compare it with the critical value $Z_{\alpha^{+}}$. If $\hat{Z}_{0}^{+}(n)>Z_{\alpha^{+}}$then the homoscedastic random walk hypothesis is rejected. This can result from either heteroscedasticity and/or autocorrelation in the time series. If $\hat{Z}_{*}^{+}(n)>Z_{\alpha^{+}}$then the random walk hypothesis is rejected and we can conclude that the data is autocorrelated.

\section{Electricity Markets Data}

To test for a random walk in electricity prices we use market data from the PJM Interconnection which is a regional transmission organization that coordinated transactions is realtime and day-ahead forward markets. Locational marginal-cost pricing (LMP) methodology, which allows prices to reflect supply and demand, as well as other characteristics of the system such as transmission lines constraints, is used to set electricity prices in the PJM. Since June 01, 2000 
there have been two markets offered by PJM. First is the day-ahead or forward market in which hourly prices are calculated for the next operating day based on demand and generation supply bids. Second is the real-time or spot market, where PJM runs an electronic auction that sets market-clearing prices every five minutes. In both real-time and day-ahead markets transactions are settled hourly.

The data set consists of six years of hourly real-time spot prices and day-ahead forward prices from June 1, 2000 to May 31, 2006. The prices are quoted in dollars per megawatt hour, $\$ / M W h$ for each on the 24 hours for each of 2191 days in the sample. The data were retrieved from the PJM website www.pjm.com. Due to non-storability of electricity, the electricity prices behavior changes throughout the day, therefore the data set is divided into 24 separate time series, for which tests are applied separately.

Summary statistics for electricity spot and forward prices are provided in Table 4-1 and Table 4-2. All statistics vary during a day and are higher for on-peak ${ }^{9}$ hours than for off-peak hours. For most of the hours spot prices are on average higher than forward prices, though median spot prices are lower than median forward prices. Both real-time and day-ahead prices display significant excess positive skewness to the right (median is smaller than mean for all hours), high volatility (standard deviation is drastically higher than unity), and fat tails (kurtosis is notably higher than 3). High kurtosis and non-zeor skewness also signal about non-normality.

\section{Empirical Evidence}

Table 4-3 and Table 4-4 present the results of Portmanteau (Ljung-Box Q) test for the PJM electricity price returns, $\Delta \mathrm{X}_{t}=\mathrm{X}_{\mathrm{t}}-\mathrm{X}_{\mathrm{t}-1}$, where $\mathrm{X}_{\mathrm{t}}$ stands either for spot or futures prices. The Ljung-Box test results indicate strong autocorrelation for all hours in both price return series. Therefore, the null of random walk is rejected. One possible reason for rejection of the Ljung-Box test null is the potential presence of some forms of non-stationarity in the series. Non-stationarity is examined by

\footnotetext{
${ }_{9}$ On-peak hours are $8 \mathrm{am}-11 \mathrm{pm}$, while off-peak hours are $11 \mathrm{pm}-7 \mathrm{am}$
} 
the unit root tests, results of which are displayed in Table 4-5 and summarized in Table 4-6. Interestingly, that the null hypothesis can be already rejected for $\mathrm{K}=2$, therefore even all other correlation coefficients estimates were zeros, the null would be rejected for higher order of correlation, i.e. for $\mathrm{K}>2$. Presence of auto- and higher order correlation in price change series may be a signal of significant seasonal fluctuations in electricity price. Further exploration is needed; particularly consideration of seasonal effects.

According to the ADF test (see Table 4-6), 7am, 8am, and 8pm real-time prices; and 8am day-ahead prices are unit root around a deterministic trend process for $10 \%$ significance level. For the confidence level of $5 \%$ unit root with trend is not rejected for 9am and 11 am spot prices; and for 7am, 10am-11am, and 12pm forward prices. The unit root with deterministic trend null can be rejected at $5 \%$ and $1 \%$ confidence levels for all other hours. Slightly different picture is given by the ADF test for unit root around deterministic level. Real-time prices for 9am-11am, and 8pm as well as forward prices for $10 \mathrm{am}$ and $11 \mathrm{am}$ are level unit root process for $10 \%$. Under $5 \%$ of confidence, the unit root with a level can not be rejected for $6-7 \mathrm{am}$, and $10-11 \mathrm{pm}$ for real-time prices and for 8am, noon, and $10-11 \mathrm{pm}$ for forward prices. As a result, based on the ADF test, we can draw a conclusion that up to $34 \%$ of the real-time market is informational efficient; while only up to $25 \%$ of day-ahead market can be considered as efficient.

As a confirmation test the KPSS signals that only 4-6am and 2-5pm spot prices are trend stationary at $5 \%$, which is in harmony with the ADF test results. For the day-ahead prices, $6 a m$ is trend stationary according to the KPSS while this series has trend unit root at $1 \%$ of confidence. According to the KPSS forward 7am price is $5 \%$ trend stationary, however according to the ADF it has trend unit root at $5 \%$. While for the day-ahead 3-5 pm prices both the ADF and KPSS are in concord for $5 \%$ of confidence. None of the spot or forward prices is level stationary according to the KPSS. In general, according to the KPSS test more hours are non-stationary which comes in contradiction with the ADF test conclusion. 
In order to further examine the unit-root presence, the DF-GLS test as a more powerful alternative to the ADF is employed. According to columns 9-12 in Table 4-6 hours 7am, 8am, and 11am for spot prices $(12.5 \%)$ as well as hours 11am and 12pm (8.34\%) for forward prices are trend unit root at least $5 \%$ of significance. While for $5 \%$ or higher level of confidence the following prices are drift unit root: (1) 12am, 5-11am, 12pm, 8pm, and 10-11pm for spot prices (50\%); and (2) 1-9am 12am for day-ahead prices (about $42 \%$ ).

Table 4-7 and Table 4-8 report the results of the Multiple Variance Ratio test for real-time and day-ahead electricity markets. Sampling intervals of $2,3,4,5,6,7,14,21,28$, and 35 days are used for both markets due to weekly fluctuations of electricity prices ${ }^{10}$. Critical values for $m=10$ are 3.289 for $1 \%$ of confidence; 2.80 for $5 \%$; and for $10 \%$ of confidence the critical value is 2.56 . Both Table 4-7 and Table 4-8 contain the absolute values of test statistics for the homoscedastic null, Z0(n), and heteroscedastic null, $Z^{*}(n)$, for each sample interval and each hour; as well as the estimates of variance ratio, $\mathrm{V}(\mathrm{n})$, less unity for sampling period of 2 and for each hour. For the MVR test only the maximum absolute values of the test statistic are considered. For each of the row, the maximum absolute value of the test statistics is marked by one (two/three) asterisk(s) if it is significant at $1 \%(5 \% / 10 \%)$, therefore indicating if the null hypothesis of random walk is rejected.

The MVR test results for real-time prices are reported in Table 4-7. According to this test the spot price cannot be considered as homoscedastic random walk for all hours; while hours 3pm, $7 \mathrm{pm}$, and $9 \mathrm{pm}$ can be treated as random walk with heteroscedastic increments. For hours 1-2pm, and $4-5 \mathrm{pm}$ the random walk hypothesis with some form of heterogeneity cannot be rejected at $5 \%$ or $1 \%$ of confidence. Therefore, real-time electricity price series of $1 \mathrm{pm}-5 \mathrm{pm}, 7 \mathrm{pm}$, and $9 \mathrm{pm}$ (seven hours) can be treated as heteroscedastic random walk. According to Table 4-8, the null for homogeneous random walk can be safely rejected for all day-ahead prices. While the null for heteroscedastic random walk cannot be rejected for $2-8 \mathrm{pm}$ for at least $1 \%$ of confidence. Thus, there are seven hours of the day-ahead market that can be considered as heteroscedastic random 
walk processes. It is worth mentioning that for both real-time and day-ahead markets about $29 \%$ of the hour price series are informational efficient. Also for both markets, only some peak price series can be considered as random walk, while all off-peak price series are highly autocorrelated.

Lo and MacKinlay (1988, p.48) have shown that under the null hypothesis of a random walk, the variance ratio less unity is asymptotically equivalent to a weighted sum of estimates of the serial correlation coefficients, i.e. variance ratio is a linear combination of sample correlation coefficient of the price changes series, and $\hat{V}(2)=1+\hat{\rho}_{\Delta}(1)$. Compare the sample autocorrelations reported in the Table 4-3 and the values of $\hat{V}(2)-1$ exhibited in the Table 4-7 and Table 4-8 one can see that these values are equivalent to the precision of the third decimal point. Also it is worth mentioning that on average the price change series are negatively correlated, meaning that generally each price spike is followed by a price drop. This is additional evidence of mean-reverting behavior of the electricity prices and additional counter-argument for random walk process; as well as evidence that "non-random" walk price series can be modeled by an autoregressive process, at least for a short-term.

An interesting observation can be made from the estimated sample autocorrelation of the price change series. For the real-time market, it is always negative and on average is equal to 0.315 for all hours, and -0.271 for those that appear to be as random walks. On contrarily, in the day-ahead market for those hours that can be believed to follow random walk, the autocorrelation of price changes series is positive (only for hour $8 \mathrm{pm}$ it is negative, -0.0044 , while very small) and on average is 0.09 , which is one-third the magnitude of the corresponding value fro real-time market. The average sample autocorrelation for all day-ahead prices is -0.034 , which is about one-ninth the magnitude of its counterpart value from the real-time market.

Another interesting pattern arises in the maximum absolute values of test statistics. For real-time prices, the heteroscedasticity null is rejected for four hours at the sampling interval 2; for

${ }^{10}$ the MVR test was performed for sampling intervals $2,7,14,21,28$, and 35 and the results are similar 
seven hours - at sampling interval of 3; for two hours - at sampling intervals of 4 and 7 ; and for one hour - at sampling intervals of 5 and 6 . At the sampling interval of 7 only for three hours (3pm, 4pm, and 9pm), the heteroscedasticity null can be accepted; while at sampling intervals of $3,5,6$, and 14, the null is accepted for one hour. The different picture is observed for day-ahead prices, though. Here, for 16 hours the heteroscedastic null is rejected at sampling interval of 7 , and only for one hour at sampling interval of 6 . At the same time the maximum absolute values for hours $3 \mathrm{pm}$, $4 \mathrm{pm}, 5 \mathrm{pm}$, and $7 \mathrm{pm}$ are at 14 days sampling interval, and for hours $2 \mathrm{pm}, 6 \mathrm{pm}$, and $8 \mathrm{pm}$ at 7 -day sampling interval. This pattern of the maximum absolute values of test statistics raises a question about the weekly seasonality effect, which electricity prices notoriously possess. A natural extension of this work will be the application of the MVR test to seasonality-filtered price series.

Comparing the results of the MVR test with those of the DF-GLS test it can be noted that the sets of hour that according to the DF-GLS test have unit root do not intersect with those that are random walk according to the MVT test for both markets, meaning those hours that can be thought as random walk based on the MVR test do not have a unit root according to the DF-GLS test. This fact is even more fascinating in the light of the fact that random walk process is a special case of a unit root.

Caution should be taken when these tests applied to time series with seasonal cycles. Phillips and Jin (2002) demonstrate that the limiting distribution of the KPSS test statistics is invariant to seasonal dummies inclusion into test regression. The same is done by Dickey et al (1986) for Dickey-Fuller tests. Despite this Lopes (2006, p.179) shows by Monte Carlo study that "not accounting for deterministic seasonality presence leads to non-similar Dickey-Fuller test statistics, plagued with problems of spurious evidence for stationarity and a rather poor power behavior". Similarly, Demetrescu and Hassler (2007) demonstrate by Monte Carlo simulations that neglecting deterministic seasonality in the ADF test leads to overrejection of the null if the process has unit root and to overacceptance of the null when the process is persistent but stationary. Moreover, neglected seasonal effects should be caught by inclusion higher number of lags, which 
decreases the power of test. The above-stated is true for the KPSS test, though it is more stable to omitted seasonal dummies. Enders (1995) suggests seasonal pre-filtering time series with deterministic periodic cycles before testing them by unit root tests.

In general, rejection of the RW hypothesis does not imply rejection of unit root hypothesis. The key assumption for the MVR test is that time series have a unit root and that disturbances are uncorrelated. Violation to either of these will lead to rejection of random walk hypothesis. However, the presence of unit root in time series does not guarantee that MVR test will accept random walk hypothesis.

To summarize, although unit root tests and MVR test contradict each other, it seems that MVR test results are more reliable than ADF/KPSS/DG-GLS tests conclusions, since both ADF and KPSS tests produce spurious results if seasonal fluctuations are left unattended. Therefore, we can conclude that PJM real-time and day-ahead markets are only partially informational efficient.

\section{Conclusion}

In this paper a Portmanteau white noise test, unit root tests, and multivariate variance ratio random walk test are employed to test the informational efficiency of the PJM electricity real-time and day-ahead markets. The results are mixed and disagree with each other. Only seven hours all of them on-peak -- in both markets can be treated as random walks according to the MVR test, which constitutes about $29 \%$.

This study sheds some light on the economic efficiency of electricity markets. Especially, the result that electricity prices are not always can be approximated by the random walk process provides evidence against the conclusions of different studies about market efficiency (see Literature review). This has a very important impact on our understanding of electricity markets, their designs, and influence of deregulation. The fact that for some hours electricity prices are not informational efficient suggests that electricity prices for these hours can be forecasted in the short- 
run using autoregressive time series modeling methods. More on employing autoregressive techniques for electricity price modeling can be found in Weron (2006).

In addition, we discuss the misuse of unit root tests in the presence of seasonal effects. Neglecting the seasonal effect while performing the ADF-type tests leads to under-rejection of unit root hypothesis, because when deterministic seasonality is ignored the distribution of the DF test is shifted to the left. On the contrary the effect of ignoring the seasonal effect on the KPSS test for stationarity depends on the estimation techniques. In general, the KPSS without seasonal effects included tends to be oversized.

There are several avenues for future research. First, incorporating deterministic seasonal effects into the unit root tests would improve our decision making on price dynamics, along with our ability to make prediction. Second, filtering out seasonal fluctuations would facilitate unit root testing and eliminate spurious results. Another extension would be to consider unit root at seasonal effects frequencies (weekly cycle for daily observations). 
Table 4-1: Summary Statistics for Real-Time Prices

\begin{tabular}{|c|c|c|c|c|c|c|c|}
\hline Hour & Min & Median & Mean & Max & $\begin{array}{c}\text { Standard } \\
\text { Deviation }\end{array}$ & $\begin{array}{c}\text { Skewness } \\
\text { Coefficient }\end{array}$ & $\begin{array}{c}\text { Kurtosis } \\
\text { Coefficient }\end{array}$ \\
\hline $\mathbf{1}$ & 1.29 & 20.65 & 24.64 & 117.57 & 13.66 & 2.05 & 8.93 \\
\hline $\mathbf{2}$ & -19.12 & 19.16 & 23.45 & 137.51 & 14.19 & 2.41 & 12.61 \\
\hline $\mathbf{3}$ & -3.21 & 17.75 & 21.57 & 122.69 & 12.81 & 2.43 & 12.10 \\
\hline $\mathbf{4}$ & -3.27 & 17.05 & 20.88 & 144.46 & 13.06 & 2.90 & 16.59 \\
\hline $\mathbf{5}$ & 0.82 & 17.74 & 22.17 & 125.07 & 13.86 & 2.63 & 12.37 \\
\hline $\mathbf{6}$ & -19.72 & 21.99 & 27.08 & 177.22 & 17.68 & 2.50 & 12.73 \\
\hline $\mathbf{7}$ & 0.06 & 29.86 & 37.95 & 251.62 & 27.72 & 1.95 & 8.53 \\
\hline $\mathbf{8}$ & 1.45 & 32.99 & 40.42 & 218.95 & 27.78 & 1.82 & 7.28 \\
\hline $\mathbf{9}$ & 2.93 & 35.38 & 40.87 & 200.65 & 23.93 & 1.75 & 7.55 \\
\hline $\mathbf{1 0}$ & 12.57 & 39.41 & 44.30 & 222.97 & 23.80 & 1.49 & 6.39 \\
\hline $\mathbf{1 1}$ & 13.28 & 43.48 & 48.49 & 247.76 & 25.42 & 1.50 & 7.29 \\
\hline $\mathbf{1 2}$ & 12.48 & 42.53 & 47.84 & 623.01 & 30.81 & 6.08 & 93.67 \\
\hline $\mathbf{1 3}$ & 12.38 & 41.50 & 47.83 & 916.68 & 38.38 & 10.54 & 209.15 \\
\hline $\mathbf{1 4}$ & 9.42 & 42.86 & 50.38 & 931.43 & 43.61 & 9.32 & 152.95 \\
\hline $\mathbf{1 5}$ & 8.53 & 38.04 & 47.08 & 931.53 & 44.42 & 9.81 & 168.04 \\
\hline $\mathbf{1 6}$ & 10.23 & 36.74 & 46.47 & 931.93 & 45.44 & 8.95 & 136.02 \\
\hline $\mathbf{1 7}$ & 11.83 & 41.81 & 50.77 & 932.27 & 45.57 & 9.05 & 145.88 \\
\hline $\mathbf{1 8}$ & 6.23 & 47.77 & 55.81 & 932.25 & 43.20 & 8.30 & 150.87 \\
\hline $\mathbf{1 9}$ & 6.81 & 43.03 & 51.39 & 801.55 & 37.39 & 6.87 & 116.65 \\
\hline $\mathbf{2 0}$ & 5.49 & 42.03 & 49.00 & 375.45 & 30.02 & 2.15 & 12.85 \\
\hline $\mathbf{2 1}$ & 13.18 & 46.16 & 51.90 & 909.08 & 34.01 & 8.35 & 189.30 \\
\hline $\mathbf{2 2}$ & 13.40 & 40.11 & 44.95 & 325.04 & 24.79 & 1.96 & 12.83 \\
\hline $\mathbf{2 3}$ & 9.47 & 28.33 & 31.90 & 117.82 & 15.92 & 1.63 & 6.53 \\
\hline $\mathbf{2 4}$ & 0.78 & 23.08 & 27.54 & 142.08 & 14.82 & 2.07 & 9.67 \\
\hline $\mathbf{J}$ & 1.2000 & & & \\
\hline
\end{tabular}

Note: June 1, 2000 to May 31, 2006 
Table 4-2: Summary Statistics for Day-Ahead Price

\begin{tabular}{|c|c|c|c|c|c|c|c|}
\hline Hour & Min & Median & Mean & Max & $\begin{array}{c}\text { Standard } \\
\text { Deviation }\end{array}$ & $\begin{array}{c}\text { Skewness } \\
\text { Coefficient }\end{array}$ & $\begin{array}{c}\text { Kurtosis } \\
\text { Coefficient }\end{array}$ \\
\hline $\mathbf{1}$ & 1.63 & 22.71 & 25.46 & 94.61 & 11.67 & 1.62 & 6.66 \\
\hline $\mathbf{2}$ & 0.66 & 19.43 & 22.40 & 85.02 & 10.50 & 1.63 & 7.02 \\
\hline $\mathbf{3}$ & 0.68 & 17.90 & 20.83 & 82.45 & 10.20 & 1.57 & 7.02 \\
\hline $\mathbf{4}$ & 0.50 & 17.19 & 20.23 & 81.45 & 10.39 & 1.60 & 7.33 \\
\hline $\mathbf{5}$ & 0.10 & 17.91 & 21.17 & 87.12 & 11.03 & 1.72 & 7.79 \\
\hline $\mathbf{6}$ & 0.10 & 22.12 & 25.76 & 105.54 & 13.66 & 1.77 & 8.00 \\
\hline $\mathbf{7}$ & 0.22 & 31.22 & 36.76 & 156.70 & 22.59 & 1.56 & 6.43 \\
\hline $\mathbf{8}$ & 0.21 & 34.72 & 40.00 & 155.71 & 21.90 & 1.42 & 5.92 \\
\hline $\mathbf{9}$ & 11.01 & 37.73 & 41.46 & 153.00 & 19.44 & 1.30 & 5.83 \\
\hline $\mathbf{1 0}$ & 12.96 & 41.69 & 44.24 & 152.94 & 18.70 & 1.09 & 5.00 \\
\hline $\mathbf{1 1}$ & 13.63 & 44.84 & 46.69 & 177.68 & 19.50 & 1.23 & 6.10 \\
\hline $\mathbf{1 2}$ & 14.05 & 44.67 & 46.81 & 320.01 & 21.30 & 2.66 & 23.97 \\
\hline $\mathbf{1 3}$ & 14.43 & 42.55 & 46.20 & 384.11 & 24.00 & 3.85 & 39.57 \\
\hline $\mathbf{1 4}$ & 14.02 & 42.17 & 46.94 & 550.01 & 28.63 & 5.95 & 79.55 \\
\hline $\mathbf{1 5}$ & 13.93 & 40.34 & 46.60 & 700.00 & 33.47 & 7.76 & 118.88 \\
\hline $\mathbf{1 6}$ & 14.00 & 39.99 & 46.95 & 701.03 & 34.66 & 7.18 & 104.50 \\
\hline $\mathbf{1 7}$ & 15.04 & 43.62 & 50.03 & 650.01 & 33.46 & 6.14 & 82.54 \\
\hline $\mathbf{1 8}$ & 15.03 & 49.62 & 56.40 & 500.01 & 31.28 & 3.61 & 35.59 \\
\hline $\mathbf{1 9}$ & 14.91 & 49.75 & 55.46 & 450.01 & 27.96 & 2.66 & 25.49 \\
\hline $\mathbf{2 0}$ & 15.12 & 48.35 & 53.24 & 356.98 & 25.87 & 2.17 & 15.68 \\
\hline $\mathbf{2 1}$ & 15.10 & 48.71 & 51.95 & 296.58 & 23.29 & 1.66 & 10.57 \\
\hline $\mathbf{2 2}$ & 15.00 & 42.34 & 44.70 & 178.78 & 19.71 & 1.34 & 6.46 \\
\hline $\mathbf{2 3}$ & 12.68 & 31.53 & 33.71 & 106.70 & 14.18 & 1.30 & 5.33 \\
\hline $\mathbf{2 4}$ & 1.38 & 25.73 & 28.41 & 96.89 & 12.46 & 1.39 & 5.54 \\
\hline $\mathbf{J u}$ & 1.2000 & & & & \\
\hline
\end{tabular}

Note: June 1, 2000 to May 31, 2006 
Table 4-3: Ljung-Box (White Noise) Q-statistics for Day-Ahead Electricity Prices Returns.

\begin{tabular}{|c|c|c|c|c|c|c|c|}
\hline & \multicolumn{6}{|c|}{ Returns of Day-Ahead Prices $\Delta \mathrm{F}_{\mathrm{t}}=\mathrm{F}_{\mathrm{t}}-\mathrm{F}_{\mathrm{t}-1}$} & $\rho(1)$ \\
\hline Hour & 2 & $\overline{7}$ & $\overline{14}$ & 21 & 28 & 35 & \\
\hline 1 & 118.44 & 192.5 & 14.6 & 260.3 & 298.3 & 330 & -0.0447 \\
\hline 2 & 34.1 & 203.7 & 241.9 & 305.4 & 356.8 & 385 & -0.0497 \\
\hline 3 & & 199 & 54 & 324 & 36 & 402 & \\
\hline 4 & & 214.9 & & 42.6 & 387.2 & 424 & \\
\hline 5 & 39 & 277.3 & .9 & 455.3 & 511.4 & 566 & \\
\hline 6 & & 564.6 & 5 & 56 & 1419 & 1678 & 07 \\
\hline 7 & & 5 & 0 & 51 & 992 & 4915 & \\
\hline 8 & & 3 & & 7 & 103 & 5012 & \\
\hline 9 & & .2 & & 5 & 2730 & 3306 & \\
\hline 10 & & 7 & 1 & 312 & 2301 & 2773 & \\
\hline 11 & & 4 & 1089 & 16 & 1919 & 2320 & -0 . \\
\hline & & 429.8 & 727 & 66.2 & 1224 & 1468 & -0.0974 \\
\hline 13 & & 418.7 & 43.5 & 824.4 & 982.9 & 1125 & -0.0058 \\
\hline 14 & & 458.6 & 646 & 785.2 & 911.1 & 1016 & 0.0589 \\
\hline 15 & & 559.9 & 96.4 & 794.4 & 871.7 & 932 & 0.1589 \\
\hline 16 & & 564.6 & 703.8 & 794.9 & 863.9 & 920 & 0.1692 \\
\hline 17 & 121.08 & 510.8 & 657.5 & 741.7 & 806 & 873 & 0.1412 \\
\hline 18 & & 466.8 & 631.5 & 738 & 811.4 & 892 & 0.0879 \\
\hline 19 & 28 & 334.9 & 437.4 & 517.6 & 583.9 & 649 & 0.0150 \\
\hline 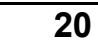 & & 318.3 & 442.5 & 538.3 & 609.6 & 682 & -0.0051 \\
\hline 21 & & 254.8 & 351.9 & 434.5 & 497.1 & 553 & -0.0801 \\
\hline 28 & & 214.6 & 296.9 & 361.6 & 403 & 447 & -0.0723 \\
\hline 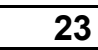 & & & 203.8 & 225.6 & 242 & 260 & -0.1237 \\
\hline 24 & 132.92 & 201.4 & 242 & 277.8 & 311.8 & 340 & -0.1113 \\
\hline
\end{tabular}

Note: Sampling interval $(\mathrm{K})$ is in days. (1) stands for sample autocorrelation of price returns

Critical values for $\mathrm{K}=2$ : $4.605(10 \%), 5.991(5 \%), 9.210(1 \%), K=7: \quad 12.017(10 \%)$, $14.067(5 \%), \quad 18.475(1 \%) ; \quad K=14: \quad 21.064(10 \%), 23.685$ (5\%), 29.141(1\%), K=21: 29.615(10\%), 32.671(5\%), 38.932(1\%); $\mathrm{K}=28: 37.916(10 \%), 41.337(5 \%), 48.278(1 \%)$; $\mathrm{K}=35: 46.059(10 \%), 49.802(5 \%), 57.342(1 \%)$ 
Table 4-4: Ljung-Box (White Noise) Q-statistics for Real-Time Electricity Prices Returns.

\begin{tabular}{|c|c|c|c|c|c|c|c|}
\hline & \multicolumn{6}{|c|}{ Returns of Real-Time Prices $\Delta \mathbf{S}_{\mathrm{t}}=\mathrm{S}_{\mathrm{t}}-\mathrm{S}_{\mathrm{t}-1}$} & $\rho(1)$ \\
\hline Hour & 2 & $\overline{7}$ & 14 & 21 & 28 & 35 & \\
\hline 1 & 290.5 & 320 & 360 & 397.7 & 415 & 443 & -0.3357 \\
\hline 2 & 90.6 & 310.6 & 332 & 363.7 & 398 & 421 & -0.3252 \\
\hline 3 & 7 & 14 & 336 & & 397 & 6 & 72 \\
\hline 4 & 307.9 & 324.8 & 334 & 35 & 367 & 387 & 262 \\
\hline 5 & 303.2 & 8.3 & 404 & $\overline{432}$ & 460 & 476 & \\
\hline 6 & 251.5 & .3 & 343 & 8 & 30 & 492 & \\
\hline 7 & 238.3 & 2.5 & 463 & & 38 & 879 & \\
\hline- & 320.1 & 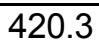 & 573 & 692.4 & 01 & 911 & \\
\hline 9 & 0 & & 559 & 26 & 711 & 768 & 73 \\
\hline 10 & 31.4 & 3.1 & 603 & 691.6 & 770 & 879 & -0. \\
\hline 11 & 352.2 & 3.8 & 515 & 579 & 676 & 786 & -0.3950 \\
\hline 12 & 230.6 & 254.7 & 273 & 330.1 & 359 & 418 & -0.2794 \\
\hline 13 & 188.8 & 202.5 & 208 & 253.6 & 261 & 289 & -0.1696 \\
\hline 14 & 76.2 & 144.3 & 177 & 262.3 & 278 & 303 & -0.1360 \\
\hline 15 & 127 & 226.3 & 330 & 410.5 & 437 & 454 & -0.2406 \\
\hline 16 & 40.2 & 261 & 271 & 366.6 & 372 & 388 & -0.2745 \\
\hline 17 & 189.2 & 223.6 & 249 & 377.8 & 381 & 394 & -0.1975 \\
\hline 18 & 137.2 & 169.5 & 194 & 251.9 & 272 & 277 & -0.1983 \\
\hline 19 & 431.7 & 457 & 472 & 485.7 & 512 & 518 & -0.4428 \\
\hline 20 & 310.4 & 334.2 & 366 & 391.1 & 458 & 481 & -0.3706 \\
\hline 21 & 388.2 & 400.3 & 410 & 431.6 & 440 & 453 & -0.4207 \\
\hline 22 & 279.4 & 294.3 & 313 & 343.2 & 356 & 359 & -0.3365 \\
\hline 23 & & 260.2 & 280 & 293.5 & 302 & 308 & -0.3107 \\
\hline 24 & 246.8 & 273.3 & 306 & 352.9 & 378 & 438 & -0.2787 \\
\hline
\end{tabular}

Note: Sampling interval $(\mathrm{K})$ is in days. (1) stands for sample autocorrelation of price returns

Critical values for $\mathbf{K}=2$ : $4.605(10 \%), 5.991(5 \%), 9.210(1 \%), K=7: \quad 12.017(10 \%)$, 14.067(5\%), 18.475(1\%); K=14: 21.064(10\%), 23.685 (5\%), 29.141(1\%), K=21: 29.615(10\%), 32.671(5\%), 38.932(1\%); $\mathrm{K}=28: 37.916(10 \%), 41.337(5 \%), 48.278(1 \%)$; $\mathrm{K}=35: 46.059(10 \%), 49.802(5 \%), 57.342(1 \%)$ 
Table 4-5: Unit Root Tests Statistics Electricity Prices

\begin{tabular}{|c|c|c|c|c|c|c|c|c|c|c|c|c|}
\hline \multirow[b]{3}{*}{ Hour } & \multicolumn{4}{|c|}{ ADF } & \multicolumn{4}{|c|}{ KPSS } & \multicolumn{4}{|c|}{ DF-GLS } \\
\hline & \multicolumn{2}{|c|}{ Trend + Intercept } & \multicolumn{2}{|c|}{ Intercept } & \multicolumn{2}{|c|}{ Trend + Intercept } & \multicolumn{2}{|c|}{ Intercept } & \multicolumn{2}{|c|}{ Trend + Intercept } & \multicolumn{2}{|c|}{ Intercept } \\
\hline & $\mathbf{R T}$ & DA & RT & DA & RT & DA & RT & DA & RT & DA & RT & DA \\
\hline 1 & $-4.460^{*}$ & $-4.319^{*}$ & $-3.042^{\star *}$ & $-3.120^{\star *}$ & $0.207^{\star *}$ & $0.189^{* *}$ & $3.558^{*}$ & $3.274^{*}$ & $-3.758^{*}$ & $-4.250^{*}$ & $-2.402^{* *}$ & -1.503 \\
\hline 2 & $-4.452^{*}$ & $-4.144^{*}$ & $-3.333^{* *}$ & $-2.928^{* *}$ & $0.148^{* *}$ & $0.231^{*}$ & $2.944^{*}$ & $3.316^{*}$ & $-4.244^{*}$ & $-4.126^{\star}$ & $-2.237^{* *}$ & $1.622^{* \star *}$ \\
\hline 3 & $-4.359^{*}$ & $001^{*}$ & $343^{* *}$ & $-2.937^{* *}$ & $181^{* *}$ & $1^{*}$ & $2.838^{*}$ & $3.108^{*}$ & $-4.242^{*}$ & -3. & $-2.057^{* *}$ & -1.559 \\
\hline 4 & $-4.252^{*}$ & ** & $*$ & & $144^{* \star \star}$ & & $2.499^{*}$ & $2.817^{*}$ & & & $-2.409^{* *}$ & *** \\
\hline 5 & $-3.936^{* *}$ & $-3.727^{* *}$ & $-3.124^{* *}$ & $-3.006^{* *}$ & $0.120^{* * *}$ & $0.162^{* *}$ & $2.788^{*}$ & $2.635^{*}$ & & & -1.173 & $34^{* * *}$ \\
\hline 6 & $-3.455^{\star *}$ & $-3.689^{* *}$ & $-2.641^{* * *}$ & $-3.076^{* *}$ & $0.139^{* * *}$ & 0.11 & $3.350^{*}$ & $2.467^{*}$ & $-3.313^{* *}$ & -3. & -1.196 & $-1.679^{* * *}$ \\
\hline 7 & -2.91 & -3.1 & $-2.686^{* * *}$ & $-2.998^{* *}$ & $0.163^{* *}$ & $0.121^{* * *}$ & $2.670^{*}$ & $1.661^{*}$ & -2.536 & $-2.997^{* *}$ & $-1.667^{* * *}$ & $-1.620^{\star * *}$ \\
\hline 8 & 078 & & $-2.803^{* *}$ & $-2.779^{* * *}$ & $177^{\star *}$ & & 2.3 & & $* *$ & & -1.256 & $6^{* \star *}$ \\
\hline 9 & $-3.347^{* * *}$ & $-3.468^{* *}$ & -2.414 & $-2.923^{* *}$ & $0.254^{*}$ & & $3.566^{*}$ & $2.484^{*}$ & & & 1.026 & -1.385 \\
\hline 10 & $-3.503^{* *}$ & $-3.171^{* * *}$ & -2.534 & -2.399 & $0.282^{*}$ & 0. & 3.5 & $2.897^{*}$ & $9^{* *}$ & -3 & -0.907 & $-1.946^{* *}$ \\
\hline 11 & $-3.168^{* * *}$ & $-3.138^{* * *}$ & -2.328 & -2.51 & $213^{* *}$ & $9^{*}$ & $2.984^{*}$ & $2.792^{*}$ & $-2.727^{* * *}$ & -2.87 & -0.696 & $-2.147^{* *}$ \\
\hline 12 & -3. & -3.3 & $-3.451^{*}$ & $-2.683^{* * *}$ & ** & $6^{*}$ & $2.525^{\star}$ & $2.534^{*}$ & $91^{*}$ & $D^{* \star *}$ & $-1.756^{* * *}$ & $-2.899^{*}$ \\
\hline 13 & -4 & -3 & -3.4 & $-2.980^{* *}$ & 0.17 & $* *$ & $1.992^{*}$ & $2.034^{*}$ & $21^{*}$ & $7^{* *}$ & $-2.334^{\star *}$ & $-2.940^{*}$ \\
\hline 14 & $-4.046^{*}$ & $-3.462^{\star *}$ & $-3.669^{*}$ & $-3.240^{\star *}$ & $0.140^{\star * *}$ & $0.163^{* *}$ & $1.201^{*}$ & $1.440^{*}$ & $-3.902^{*}$ & & $-2.899^{*}$ & $-3.234^{*}$ \\
\hline 15 & $-3.799^{* *}$ & $-3.531^{* *}$ & $-3.466^{*}$ & $-3.367^{* *}$ & $0.133^{* * *}$ & $0.132^{\star * *}$ & $1.001^{*}$ & $1.056^{*}$ & $-3.563^{*}$ & $-3.323^{* *}$ & $-2.658^{*}$ & $-3.337^{*}$ \\
\hline 16 & $-3.633^{* *}$ & $17^{* *}$ & $-3.201^{* *}$ & $-3.376^{\star *}$ & $0.121^{\text {***}}$ & $D^{* * *}$ & $1.080^{*}$ & $0.932^{*}$ & $-3.286^{* *}$ & & $-2.972^{*}$ & $-3.355^{*}$ \\
\hline 17 & -4 & -3 & -3.6 & -3 & $0.130^{* * *}$ & $* * *$ & 1.2 & $8^{*}$ & $47^{*}$ & $4^{* *}$ & $37^{*}$ & $-3.142^{*}$ \\
\hline 18 & $-4.405^{\star}$ & $-4.333^{*}$ & $-3.885^{*}$ & $-3.911^{*}$ & $0.166^{\star *}$ & $0.169^{* *}$ & $1.531^{*}$ & $1.255^{*}$ & $-3.855^{\star}$ & $-4.238^{*}$ & $-3.828^{*}$ & $-3.627^{*}$ \\
\hline 19 & $-4.168^{*}$ & $-3.886^{* *}$ & $-3.438^{*}$ & $-3.382^{* *}$ & $0.218^{*}$ & $0.213^{* *}$ & $2.403^{*}$ & $1.836^{*}$ & $-3.270^{\star *}$ & $-3.886^{*}$ & $-3.408^{*}$ & $-2.625^{*}$ \\
\hline 20 & 06 & $-3.488^{* *}$ & -2.488 & $-3.000^{* *}$ & $0.245^{\star}$ & $4^{*}$ & $2.807^{*}$ & $2.230^{*}$ & -3.0 & & 155 & $-2.541^{* *}$ \\
\hline 21 & $-4.486^{*}$ & $-3.412^{* *}$ & $-3.501^{*}$ & $-2.909^{* *}$ & $0.255^{\star}$ & $0.324^{*}$ & $2.852^{*}$ & $2.689^{*}$ & $-4.357^{*}$ & $4^{* *}$ & $-1.989^{* *}$ & $-2.680^{*}$ \\
\hline 22 & $-4.028^{*}$ & $-3.449^{* *}$ & $-2.827^{* * *}$ & $-2.686^{\star * *}$ & $0.289^{*}$ & $0.321^{*}$ & $3.352^{*}$ & $3.085^{*}$ & $-3.894^{*}$ & $-3.200^{* *}$ & $-1.635^{\star * *}$ & $-2.478^{* *}$ \\
\hline 23 & $-3.963^{*}$ & $-3.946^{* *}$ & $-2.809^{* * *}$ & $-2.737^{* * *}$ & $0.359^{*}$ & $0.325^{*}$ & $3.507^{*}$ & $3.263^{*}$ & $-3.884^{*}$ & $-3.825^{\star}$ & $-1.868^{* * *}$ & $-2.062^{* *}$ \\
\hline 24 & $-4.375^{*}$ & $-4.414^{*}$ & $-2.871^{* *}$ & $-3.250^{\star *}$ & $0.305^{*}$ & $0.274^{*}$ & $3.900^{*}$ & $3.272^{*}$ & $-4.334^{*}$ & $-4.344^{*}$ & $-1.710^{\star * *}$ & -1.531 \\
\hline
\end{tabular}

Note: Real-time (RT) and Day-Ahead (DA), 
Table 4-6: Summary of Unit Root Tests Results for Electricity Prices

\begin{tabular}{|c|c|c|c|c|c|c|c|c|c|c|c|c|}
\hline & \multicolumn{4}{|c|}{ ADF } & \multicolumn{4}{|c|}{ KPSS } & \multicolumn{4}{|c|}{ DF-GLS } \\
\hline & \multicolumn{2}{|c|}{$\begin{array}{c}\text { Trend + } \\
\text { Intercept }\end{array}$} & \multicolumn{2}{|c|}{ Intercept } & \multicolumn{2}{|c|}{$\begin{array}{c}\text { Trend + } \\
\text { Intercept }\end{array}$} & \multicolumn{2}{|c|}{ Intercept } & \multicolumn{2}{|c|}{$\begin{array}{c}\text { Trend + } \\
\text { Intercept }\end{array}$} & \multicolumn{2}{|c|}{ Intercept } \\
\hline Hour & RT & DA & RT & DA & RT & DA & RT & DA & RT & DA & RT & DA \\
\hline 1 & No UR & No UR & No UR & No UR & UR & UR & UR & UR & No UR & No UR & $S$ & UR \\
\hline 2 & No UR & No UR & No UR & No UR & UR & UR & UR & UR & No UR & No UR & No UR & UR \\
\hline 3 & No UR & No UR & No UR & No UR & UR & UR & UR & UR & No UR & No UR & No UR & UR \\
\hline 4 & No UR & No UR & No UR & No UR & $S$ & UR & UR & UR & No UR & No UR & No UR & UR \\
\hline 5 & No UR & No UR & No UR & No UR & $S$ & UR & UR & UR & No UR & No UR & UR & UR \\
\hline 6 & No UR & No UR & UR & No UR & $S$ & $S$ & UR & UR & No UR & No UR & UR & UR \\
\hline 7 & UR & UR & UR & No UR & UR & $S$ & UR & UR & UR & No UR & UR & UR \\
\hline 8 & UR & UR & UR & UR & UR & UR & UR & UR & UR & No UR & UR & UR \\
\hline 9 & UR & No UR & UR & No UR & UR & UR & UR & UR & No UR & No UR & UR & UR \\
\hline 10 & No UR & UR & UR & UR & UR & UR & UR & UR & No UR & No UR & UR & No UR \\
\hline 11 & UR & UR & UR & UR & UR & UR & UR & UR & UR & UR & UR & No UR \\
\hline 12 & No UR & UR & No UR & UR & UR & UR & UR & UR & No UR & UR & UR & No UR \\
\hline 13 & No UR & No UR & No UR & No UR & UR & UR & UR & UR & No UR & No UR & No UR & No UR \\
\hline 14 & No UR & No UR & No UR & No UR & $S$ & UR & UR & UR & No UR & No UR & No UR & No UR \\
\hline 15 & No UR & No UR & No UR & No UR & $\mathrm{S}$ & $S$ & UR & UR & No UR & No UR & No UR & No UR \\
\hline 16 & No UR & No UR & No UR & No UR & $S$ & $S$ & UR & UR & No UR & No UR & No UR & No UR \\
\hline 17 & No UR & No UR & No UR & No UR & $S$ & $S$ & UR & UR & No UR & No UR & No UR & No UR \\
\hline 18 & No UR & No UR & No UR & No UR & UR & UR & UR & UR & No UR & No UR & No UR & No UR \\
\hline 19 & No UR & No UR & No UR & No UR & UR & UR & UR & UR & No UR & No UR & No UR & No UR \\
\hline 20 & UR & No UR & UR & No UR & UR & UR & UR & UR & No UR & No UR & UR & No UR \\
\hline 21 & No UR & No UR & No UR & No UR & UR & UR & UR & UR & No UR & No UR & No UR & No UR \\
\hline 22 & No UR & No UR & UR & UR & UR & UR & UR & UR & No UR & No UR & UR & No UR \\
\hline 23 & No UR & No UR & UR & UR & UR & UR & UR & UR & No UR & No UR & UR & No UR \\
\hline 24 & No UR & No UR & No UR & No UR & UR & UR & UR & UR & No UR & No UR & UR & UR \\
\hline
\end{tabular}

Note: Real-time (RT) and Day-Ahead (DA). Confidence is $95 \%$

UR stands fro "Unit Root Hypothesis is accepted', No UR stands for "Unit Root Hypothesis is not accepted",

$S$ stands for "Hypothesis of Stationarity is accepted" 
Table 4-7: Multivariate Variance Ratio Test for Real-Time Prices.

\begin{tabular}{|c|c|c|c|c|c|c|c|c|c|c|c|c|}
\hline Hour & $V(2)-1$ & & 2 & 3 & 4 & 5 & 6 & 7 & 14 & 21 & 28 & 35 \\
\hline \multirow{2}{*}{1} & \multirow{2}{*}{-0.336} & $\mathrm{ZO}(\mathrm{n})$ & 15.73 & $17.02^{*}$ & 16.01 & 14.7 & 13.96 & 13.49 & 10.05 & 8.38 & 7.34 & 6.62 \\
\hline & & $\mathbf{Z}^{*}(n)$ & 7.18 & $8.19^{*}$ & 8.09 & 7.73 & 7.56 & 7.49 & 6.18 & 5.44 & 4.94 & 4.56 \\
\hline \multirow{2}{*}{2} & \multirow{2}{*}{-0.325} & $\mathrm{ZO}(\mathrm{n})$ & 15.21 & $17.03^{*}$ & 16.29 & 15.07 & 14.38 & 13.7 & 10.08 & 8.39 & 7.34 & 6.62 \\
\hline & & $Z^{*}(n)$ & 6.43 & 7.61 & $7.62^{*}$ & 7.27 & 7.1 & 6.88 & 5.39 & 4.67 & 4.24 & 3.95 \\
\hline \multirow{2}{*}{3} & \multirow{2}{*}{-0.307} & $\mathrm{ZO}(\mathrm{n})$ & 14.37 & $17.02^{*}$ & 16.16 & 15.03 & 14.25 & 13.67 & 9.98 & 8.34 & 7.3 & 6.59 \\
\hline & & $Z^{*}(n)$ & 5.9 & $7.31^{\star}$ & 7.24 & 6.96 & 6.78 & 6.64 & 5.19 & 4.52 & 4.06 & 3.75 \\
\hline \multirow{2}{*}{4} & \multirow{2}{*}{-0.326} & $\mathrm{ZO}(\mathrm{n})$ & 15.24 & $17.50^{*}$ & 16.29 & 14.94 & 14.26 & 13.54 & 9.95 & 8.34 & 7.29 & 6.58 \\
\hline & & $Z^{*}(n)$ & 6.26 & $7.22^{*}$ & 6.85 & 6.43 & 6.28 & 6.08 & 4.84 & 4.29 & 3.91 & 3.64 \\
\hline \multirow{2}{*}{5} & \multirow{2}{*}{-0.340} & $Z 0(n)$ & 15.93 & $17.37^{*}$ & 16.36 & 15.25 & 14.59 & 13.85 & 10.14 & 8.45 & 7.36 & 6.63 \\
\hline & & $Z^{*}(n)$ & 6.81 & $7.72^{*}$ & 7.57 & 7.31 & 7.21 & 7.01 & 5.49 & 4.73 & 4.23 & 3.89 \\
\hline \multirow{2}{*}{6} & \multirow{2}{*}{-0.312} & $Z 0(n)$ & 14.62 & $15.83^{*}$ & 15.38 & 15.11 & 14.55 & 13.97 & 10.14 & 8.48 & 7.4 & 6.67 \\
\hline & & $Z^{*}(n)$ & 7.43 & 8.21 & 8.18 & $8.25^{*}$ & 8.13 & 7.96 & 6.28 & 5.5 & 4.9 & 4.47 \\
\hline \multirow{2}{*}{7} & \multirow{2}{*}{-0.296} & $Z 0(n)$ & 13.87 & $15.48^{*}$ & 15.03 & 14.98 & 14.89 & 14.5 & 10.44 & 8.63 & 7.5 & 6.75 \\
\hline & & $Z^{*}(n)$ & 8.53 & 9.68 & 9.59 & 9.73 & $9.81^{*}$ & 9.67 & 7.16 & 5.99 & 5.24 & 4.75 \\
\hline \multirow{2}{*}{8} & \multirow{2}{*}{-0.374} & $Z 0(n)$ & $17.52^{*}$ & 17.26 & 16.32 & 15.5 & 14.95 & 14.53 & 10.52 & 8.63 & 7.52 & 6.75 \\
\hline & & $Z^{*}(n)$ & 10.24 & $10.47^{*}$ & 10.23 & 9.95 & 9.77 & 9.62 & 7.28 & 6.09 & 5.37 & 4.88 \\
\hline \multirow{2}{*}{9} & \multirow{2}{*}{-0.437} & $Z 0(n)$ & $20.45^{\star}$ & 18.67 & 17.23 & 16.11 & 14.86 & 14.31 & 10.41 & 8.59 & 7.5 & 6.73 \\
\hline & & $\mathbf{Z}^{*}(n)$ & $10.83^{*}$ & 10.43 & 10.11 & 9.84 & 9.38 & 9.28 & 7.5 & 6.48 & 5.8 & 5.28 \\
\hline 10 & -0442 & $Z 0(n)$ & $20.68^{*}$ & 19.29 & 17.51 & 16.14 & 15.07 & 14.53 & 10.5 & 8.63 & 7.53 & 6.75 \\
\hline 10 & -0.442 & $Z^{*}(n)$ & $11.78^{*}$ & 11.59 & 11.04 & 10.6 & 10.23 & 10.13 & 8.04 & 6.8 & 6.01 & 5.44 \\
\hline & & $Z 0(n)$ & $18.49^{*}$ & 17.96 & 16.88 & 15.59 & 14.75 & 14.28 & 10.43 & 8.6 & 7.49 & 6.72 \\
\hline 11 & -0.395 & $\mathbf{Z}^{*}(n)$ & 11.79 & $12.12^{*}$ & 11.92 & 11.39 & 11.04 & 10.88 & 8.41 & 7.09 & 6.24 & 5.63 \\
\hline & & $Z 0(n)$ & 13.09 & $15.13^{*}$ & 14.85 & 14.11 & 13.59 & 13.27 & 10.13 & 8.43 & 7.38 & 6.65 \\
\hline 12 & -0.28 & $\mathbf{Z}^{*}(n)$ & $6.20^{*}$ & 4.66 & 4.13 & 3.89 & 3.82 & 3.84 & 3.57 & 3.34 & 3.12 & 2.96 \\
\hline 13 & -0170 & $Z 0(n)$ & 7.97 & 12.11 & $12.51^{*}$ & 12.44 & 12.47 & 12.21 & 9.79 & 8.26 & 7.26 & 6.57 \\
\hline & $-0.1 / 0$ & $Z^{*}(n)$ & 2.42 & $2.58^{* \star \star}$ & 2.42 & 2.39 & 2.44 & 2.46 & 2.45 & 2.35 & 2.23 & 2.14 \\
\hline & & $Z 0(n)$ & 6.49 & 8.4 & 9.56 & 10.68 & 11.22 & $11.38^{*}$ & 9.67 & 8.11 & 7.16 & 6.49 \\
\hline 14 & -0.13 & $Z^{*}(n)$ & 1.4 & 1.95 & 2.31 & 2.62 & 2.81 & 2.92 & $2.98^{* *}$ & 2.71 & 2.45 & 2.29 \\
\hline 15 & (210 & $Z 0(n)$ & 11.25 & 9.98 & 10.99 & 11.7 & $12.17^{*}$ & 12.08 & 9.91 & 8.22 & 7.25 & 6.54 \\
\hline 15 & $-0 . \angle 40$ & $\mathbf{Z}^{*}(n)$ & 1.91 & 1.77 & 1.93 & 2.05 & 2.16 & 2.19 & 2.17 & 1.98 & 1.86 & 1.77 \\
\hline 16 & 0 & $Z 0(n)$ & 13.08 & $15.46^{*}$ & 15.32 & 14.6 & 14.3 & 13.58 & 10.34 & 8.5 & 7.43 & 6.7 \\
\hline 16 & -0.219 & $Z^{*}(n)$ & 1.88 & 2.33 & 2.45 & 2.5 & 2.6 & $2.61^{\star \star \star}$ & 2.61 & 2.48 & 2.37 & 2.28 \\
\hline 17 & 2020 & $Z 0(n)$ & 9.47 & 12.91 & 13.51 & 13.48 & $13.57^{*}$ & 12.96 & 10.21 & 8.4 & 7.35 & 6.62 \\
\hline 17 & & $Z^{*}(n)$ & 2.68 & 2.81 & 2.75 & 2.77 & $2.87^{* \star}$ & 2.83 & 2.73 & 2.49 & 2.29 & 2.17 \\
\hline 18 & 198 & $Z 0(n)$ & 9.25 & 11.47 & 12.63 & $12.81^{*}$ & 12.75 & 12.44 & 9.95 & 8.25 & 7.26 & 6.55 \\
\hline 10 & & $Z^{*}(n)$ & $4.38^{*}$ & 3.11 & 2.99 & 2.95 & 2.97 & 2.97 & 2.89 & 2.72 & 2.59 & 2.5 \\
\hline 19 & -0.442 & $\mathrm{ZO}(\mathrm{n})$ & $20.71^{*}$ & 19.12 & 17.11 & 16.32 & 15.15 & 14.14 & 10.44 & 8.58 & 7.49 & 6.72 \\
\hline & & $\mathbf{Z}^{*}(\mathrm{n})$ & 2.38 & 2.45 & 2.43 & 2.54 & 2.54 & 2.53 & 2.52 & 2.47 & 2.43 & 2.4 \\
\hline 20 & 370 & $Z 0(n)$ & $17.32^{*}$ & 16.85 & 15.99 & 15.38 & 14.65 & 14.11 & 10.34 & 8.53 & 7.45 & 6.68 \\
\hline 20 & -1 & $\mathbf{Z}^{*}(n)$ & 6.02 & 6.4 & 6.59 & 6.77 & 6.81 & $6.87^{*}$ & 6.09 & 5.52 & 5.09 & 4.74 \\
\hline 21 & -0421 & $Z 0(n)$ & $19.70^{*}$ & 17.66 & 16.34 & 15.29 & 14.55 & 13.89 & 10.25 & 8.52 & 7.44 & 6.68 \\
\hline & & $Z^{*}(n)$ & 1.86 & 1.84 & 1.87 & 1.89 & 1.93 & 1.96 & 1.95 & 1.95 & 1.94 & 1.93 \\
\hline 22 & 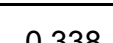 & $Z 0(n)$ & 15.83 & $16.61^{*}$ & 16.05 & 15.27 & 14.3 & 13.75 & 10.2 & 8.48 & 7.38 & 6.65 \\
\hline 22 & 338 & $\mathbf{Z}^{*}(n)$ & 5.46 & 6.27 & 6.6 & 6.74 & 6.69 & $6.75^{*}$ & 6.13 & 5.62 & 5.17 & 4.84 \\
\hline 23 & -0310 & $Z 0(n)$ & 14.52 & $15.86^{*}$ & 15.01 & 14.49 & 13.94 & 13.37 & 10.04 & 8.39 & 7.34 & 6.62 \\
\hline & -0.310 & $\mathbf{Z}^{*}(n)$ & 7.64 & $8.77^{*}$ & 8.69 & 8.69 & 8.59 & 8.41 & 6.79 & 5.86 & 5.21 & 4.76 \\
\hline 21 & & $Z 0(n)$ & 13.02 & $15.55^{*}$ & 15.37 & 14.67 & 13.99 & 13.57 & 10.08 & 8.4 & 7.35 & 6.64 \\
\hline 24 & -0.21 & $Z^{*}(n)$ & 5.56 & 7 & $7.28^{*}$ & 7.23 & 7.11 & 7.07 & 5.67 & 4.89 & 4.39 & 4.04 \\
\hline
\end{tabular}

Note: Critical values for MVR test are 3.289 at $1 \%, 2.7996$ at $5 \%$, and 2.5596 at $10 \%$, for $m=10 .{ }^{*}$ - $1 \%$ significance; ** --- $5 \%$ significance, and ${ }^{* * *}--10 \%$ significance. $V(2)-1=\rho(1)$ stands for sample autocorrelation of price returns. Sampling intervals $(n)$ are in days 
Table 4-8: Multiple Variance Ratio Test for Day-Ahead Prices.

\begin{tabular}{|c|c|c|c|c|c|c|c|c|c|c|c|c|}
\hline Hour & $V(2)-1$ & & 2 & 3 & 4 & 5 & 6 & 7 & 14 & 21 & 28 & 35 \\
\hline \multirow[b]{2}{*}{1} & \multirow[b]{2}{*}{-0.044} & ZO(n) & 2.06 & 6.63 & 8.75 & 9.52 & 10.08 & $10.13^{*}$ & 8.14 & 7.12 & 6.43 & 5.96 \\
\hline & & $Z^{*}(n)$ & 0.9 & 2.96 & 3.97 & 4.38 & 4.68 & $4.74^{*}$ & 4.02 & 3.67 & 3.44 & 3.29 \\
\hline \multirow[b]{2}{*}{2} & \multirow[b]{2}{*}{-0.049} & ZO(n) & 2.3 & 7.13 & 9.26 & 9.94 & 10.32 & $10.37^{*}$ & 8.2 & 7.17 & 6.44 & 5.98 \\
\hline & & $Z^{*}(n)$ & 0.96 & 3.03 & 4 & 4.37 & 4.63 & $4.72^{*}$ & 3.96 & 3.63 & 3.38 & 3.22 \\
\hline \multirow[b]{2}{*}{3} & \multirow[b]{2}{*}{-0.057} & ZO(n) & 2.67 & 7.1 & 9.03 & 9.94 & 10.41 & $10.45^{*}$ & 8.25 & 7.21 & 6.46 & 6 \\
\hline & & $Z^{*}(n)$ & 1.08 & 2.92 & 3.77 & 4.25 & 4.55 & $4.65^{*}$ & 3.93 & 3.6 & 3.33 & 3.17 \\
\hline \multirow[b]{2}{*}{4} & \multirow[b]{2}{*}{-0.034} & ZO(n) & 1.64 & 6.58 & 8.81 & 9.92 & 10.41 & $10.46^{*}$ & 8.27 & 7.24 & 6.47 & 6 \\
\hline & & $Z^{*}(n)$ & 0.68 & 2.78 & 3.76 & 4.3 & 4.61 & $4.70^{*}$ & 3.96 & 3.64 & 3.36 & 3.19 \\
\hline \multirow[b]{2}{*}{5} & \multirow[b]{2}{*}{-0.044} & ZO(n) & 2.04 & 7.39 & 9.54 & 10.53 & 11.11 & $11.12^{*}$ & 8.54 & 7.38 & 6.57 & 6.07 \\
\hline & & $Z^{*}(n)$ & 0.97 & 3.42 & 4.38 & 4.86 & 5.17 & $5.22^{*}$ & 4.16 & 3.73 & 3.44 & 3.26 \\
\hline \multirow[b]{2}{*}{6} & \multirow[b]{2}{*}{-0.05} & ZO(n) & 2.34 & 8.36 & 10.53 & 11.59 & 12.63 & $12.83^{*}$ & 9.28 & 7.83 & 6.91 & 6.33 \\
\hline & & $Z^{*}(n)$ & 1.3 & 4.54 & 5.7 & 6.33 & 6.97 & $7.13^{*}$ & 5.23 & 4.49 & 4.04 & 3.77 \\
\hline \multirow[b]{2}{*}{7} & \multirow[b]{2}{*}{-0.037} & ZO(n) & 1.74 & 9.32 & 11.45 & 12.36 & 14.16 & $14.76^{*}$ & 10.26 & 8.41 & 7.33 & 6.63 \\
\hline & & $Z^{*}(n)$ & 1.13 & 5.88 & 7.25 & 7.94 & 9.2 & $9.66^{*}$ & 6.69 & 5.49 & 4.83 & 4.42 \\
\hline \multirow[b]{2}{*}{8} & \multirow[b]{2}{*}{-0.074} & ZO(n) & 3.48 & 10.06 & 11.9 & 12.5 & 14.06 & $14.81^{*}$ & 10.32 & 8.43 & 7.34 & 6.64 \\
\hline & & $Z^{*}(n)$ & 2.5 & 6.98 & 8.21 & 8.7 & 9.87 & $10.46^{*}$ & 7.19 & 5.85 & 5.1 & 4.64 \\
\hline \multirow[b]{2}{*}{9} & \multirow[b]{2}{*}{-0.170} & ZO(n) & 7.97 & 12.02 & 12.75 & 12.83 & 13.52 & $14.10^{*}$ & 10.03 & 8.28 & 7.24 & 6.56 \\
\hline & & $Z^{*}(n)$ & 5.16 & 7.88 & 8.57 & 8.86 & 9.53 & $10.08^{*}$ & 7.18 & 5.93 & 5.22 & 4.78 \\
\hline & & ZO(n) & 9.73 & 12.48 & 12.81 & 12.65 & 12.99 & $13.55^{*}$ & 9.9 & 8.21 & 7.2 & 6.53 \\
\hline 10 & -0.208 & $Z^{*}(n)$ & 5.98 & 8 & 8.58 & 8.79 & 9.28 & $9.86^{*}$ & 7.41 & 6.19 & 5.49 & 5.04 \\
\hline & & ZO(n) & 9.2 & 11.55 & 11.97 & 12.1 & 12.57 & $13.12^{*}$ & 9.79 & 8.16 & 7.17 & 6.5 \\
\hline 11 & -0.197 & $Z^{*}(n)$ & 5.17 & 6.77 & 7.28 & 7.55 & 7.99 & $8.46^{*}$ & 6.67 & 5.75 & 5.19 & 4.82 \\
\hline & & ZO(n) & 4.53 & 7.01 & 8.72 & 10.04 & 11.11 & $11.79^{*}$ & 9.43 & 7.95 & 7.04 & 6.4 \\
\hline 12 & -0.097 & $Z^{*}(n)$ & 1.51 & 2.44 & 3.05 & 3.47 & 3.83 & $4.10^{*}$ & 3.74 & 3.53 & 3.4 & 3.3 \\
\hline & & ZO(n) & 0.24 & 4.29 & 6.18 & 8.37 & 10.15 & $10.91^{*}$ & 9.09 & 7.77 & 6.91 & 6.3 \\
\hline 13 & -0.005 & $Z^{*}(n)$ & 0.09 & 1.52 & 2.17 & 2.77 & 3.17 & $3.31^{*}$ & 2.9 & 2.74 & 2.66 & 2.6 \\
\hline & & ZO(n) & 2.8 & 1.39 & 3.96 & 6.91 & 9.15 & $10.12^{*}$ & 8.88 & 7.66 & 6.84 & 6.23 \\
\hline 14 & 0.06 & $Z^{*}(n)$ & 0.62 & 0.3 & 0.84 & 1.44 & 1.86 & 2.05 & 2 & 1.95 & 1.92 & 1.9 \\
\hline & & ZO(n) & 7.48 & 3 & 0.94 & 5.04 & 7.75 & $8.92^{*}$ & 8.49 & 7.45 & 6.69 & 6.12 \\
\hline 15 & 0.16 & $Z^{*}(n)$ & 1.2 & 0.49 & 0.16 & 0.84 & 1.28 & 1.47 & 1.58 & 1.58 & 1.57 & 1.57 \\
\hline & & ZO(n) & 7.97 & 3.45 & 0.74 & 4.91 & 7.63 & $8.81^{*}$ & 8.47 & 7.41 & 6.65 & 6.1 \\
\hline 16 & 0.170 & $Z^{*}(n)$ & 1.33 & 0.59 & 0.13 & 0.85 & 1.31 & 1.52 & 1.65 & 1.64 & 1.63 & 1.62 \\
\hline & & ZO(n) & 6.65 & 2.03 & 1.91 & 5.66 & 8.23 & $9.26^{*}$ & 8.59 & 7.46 & 6.68 & 6.12 \\
\hline 17 & 0.142 & $Z^{*}(n)$ & 1.21 & 0.38 & 0.37 & 1.09 & 1.58 & 1.8 & 1.92 & 1.89 & 1.87 & 1.87 \\
\hline & & ZO(n) & 4.16 & 0.05 & 3.52 & 6.94 & 9.24 & $10.01^{*}$ & 8.66 & 7.43 & 6.63 & 6.07 \\
\hline 18 & 0.089 & $Z^{*}(n)$ & 1.01 & 0.01 & 0.92 & 1.83 & 2.43 & $2.65^{\star \star *}$ & 2.57 & 2.47 & 2.41 & 2.38 \\
\hline & & ZO(n) & 0.74 & 2.84 & 6.34 & 8.78 & 10.34 & $10.79^{*}$ & 8.87 & 7.58 & 6.76 & 6.18 \\
\hline 19 & 0.016 & $Z^{*}(n)$ & 0.14 & 0.56 & 1.31 & 1.87 & 2.28 & 2.47 & 2.5 & 2.47 & 2.45 & 2.43 \\
\hline & & ZO(n) & 0.21 & 3.37 & 6.76 & 9.09 & 10.46 & $10.91^{*}$ & 8.87 & 7.58 & 6.74 & 6.17 \\
\hline 20 & -0.004 & $Z^{*}(n)$ & 0.05 & 0.89 & 1.8 & 2.42 & 2.82 & $3.00^{* *}$ & 2.89 & 2.82 & 2.77 & 2.74 \\
\hline & & ZO(n) & 3.72 & 5.94 & 8.46 & 10.26 & 11.4 & $11.55^{*}$ & 9.07 & 7.73 & 6.85 & 6.27 \\
\hline 21 & -0.08 & $Z^{*}(n)$ & 0.93 & 1.58 & 2.33 & 2.91 & 3.33 & $3.49^{*}$ & 3.31 & 3.21 & 3.14 & 3.09 \\
\hline & & $Z 0(n)$ & 3.36 & 5.96 & 8.42 & 10.03 & 11.06 & $11.16^{*}$ & 8.99 & 7.67 & 6.81 & 6.25 \\
\hline 22 & -0.072 & $Z^{*}(n)$ & 1.66 & 3.08 & 4.3 & 5.04 & 5.53 & $5.61^{*}$ & 4.89 & 4.47 & 4.18 & 4.01 \\
\hline & & ZO(n) & 5.78 & 8.1 & 9.54 & 10.39 & $10.98^{*}$ & 10.97 & 8.88 & 7.59 & 6.77 & 6.21 \\
\hline 23 & -0.124 & $Z^{*}(n)$ & 2.71 & 3.97 & 4.82 & 5.36 & 5.77 & $5.84^{*}$ & 5.01 & 4.44 & 4.07 & 3.84 \\
\hline & & ZO(n) & 5.17 & 9.22 & 10.24 & 11.07 & $11.62^{*}$ & 11.39 & 8.8 & 7.49 & 6.67 & 6.12 \\
\hline 24 & -0.111 & $Z^{*}(n)$ & 2.11 & 3.89 & 4.43 & 4.85 & $5.15^{*}$ & 5.09 & 4.07 & 3.6 & 3.34 & 3.18 \\
\hline
\end{tabular}

Note: Critical values for MVR test are 3.289 at $1 \%, 2.7996$ at $5 \%$, and 2.5596 at $10 \%$, for $m=10 .{ }^{*}$ - $1 \%$ significance; ** --- 5\% significance, and ${ }^{* * *}$-- 10\% significance. V(2)-1 $=\rho(1)$ stands for sample autocorrelation of price returns. Sampling intervals $(n)$ are in days 


\section{Chapter 5 : Note on Stationarity of Electricity Prices}

\section{Introduction}

Electricity prices are characterized by volatility changes and clustering, mean-reversion, spikes and jumps, and seasonal fluctuations. In the growing literature on electricity price characteristics and modeling there is a mixed message about the stationarity of wholesale electricity price series. In this paper we: (1) review and comment on existing studies on electricity prices stationarity; (2) review the literature on controversies of classic unit root tests application to time series characterized by seasonality; and (3) examine whether PJM real-time and day-ahead prices have unit root, are S, or are covariance non-stationary without unit root.

There is huge and growing literature on statistical properties and characteristics of electricity prices in deregulated markets around the world. In the nearly exhaustive empirical analysis of restructured electricity prices Knittel and Roberts (2005, p.792) find "stationarity in both price level and squared prices" of California electricity market. In contrast, De Vany and Walls (1999b) find the presence of a unit root in on-peak and off-peak electricity price series of 11 western US electricity markets (including California). Arciniegas et al (2003), while assessing the level and degree of efficiency reached by the California, New York, and PJM real-time and day-ahead markets, find that for most hours electricity prices have a unit root. Lu and Dong (2005) detect stationarity in on-peak and off-peak real-time and futures electricity prices of the Australian National Electricity market. The same conclusion for the electricity prices of the Australian National Electricity market is reached in Worthington and Higgs (2004) and in Worthington et al (2005). Goto and Karolyi (2004) find that Dow Jones Electricity Price Index, Nord Pool daily electricity prices and demand-weighted daily electricity prices from Australian National Electricity market do not contain a robust indication of a unit root. Gjolberg (2001) finds that monthly average electricity prices from Nord Pool market are non-stationary and posses a unit root. Ferreira et al. (2005) discover that 
quarterly electricity prices in the United Kingdom are integrated of degree one (in other words have a unit root) in both residential and industrial sectors. Emery and Liu (2002) find a unit root in electricity futures prices traded at NYMEX.

By definition, a stationary time series is one with constant over time statistical properties such as mean, variance, autocorrelation, and etc. Numerous tests have been developed for stationarity including the Dickey-Fuller (ADF) test (Fuller (1976), Dickey and Fuller (1979)), DickeyFuller Generalized Least Squares (DF-GLS) test (Elliot, Rosenberg, and Stock (1996)), PhillipsPerron test (Phillips (1987), Phillips and Perron (1988)) and Kwiatkowski-Phillips-Schmidt-Shin (KPSS) test (Kwiatkowski et al. (1992)). However, caution should be taken when these tests are applied to time series with seasonal fluctuations. Phillips and Jin (2002) demonstrate that the limiting distribution of the KPSS test statistics is invariant to seasonal dummies inclusion in the test regression. In the same vein, Dickey et al (1986) and Ghysels et al (1994) show that inclusion of seasonal dummies does not affect the limiting distribution of Dickey-Fuller tests. Despite this Lopes (2006, p.179) shows by Monte Carlo study that "not accounting for deterministic seasonality presence leads to non-similar Dickey-Fuller test statistics, plagued with problems of spurious evidence for stationarity and a rather poor power behavior". Similarly, Demetrescu and Hassler (2007) demonstrate by Monte Carlo simulations that neglecting deterministic seasonality in the ADF test leads to overrejection of the null if the process has a unit root and to overacceptance of the null when the process is persistent but stationary. Moreover, neglected seasonal effects should be caught by inclusion of a higher number of lags, which decreases the power of test. The abovestated is true for the KPSS test, though it is more stable to omitted seasonal dummies. Therefore, caution should be taken when applying the ADF test results directly to electricity prices, which include strong seasonal pattern associated with time of day, day of week, and season of year. Enders (1995) suggests seasonal pre-filtering time series with deterministic periodic cycles before testing them by unit root tests. 
Another critique of Dickey-Fuller and KPSS tests is that both tests may failure to detect non-stationarity due to shifts in unconditional variance and shown by Xiao and Lima (2007), Ahamada (2004) and Liew et al (2007). In the latter two manuscripts a complementary test - based on Inclan and Tiao (1994) cumulative sums of squares procedure - is proposed to complement unit root tests. Applying this complementary test to de-seasoned electricity prices, we find that they contain heterogeneous variance but not a stochastic trend represented by a unit root.

The testing procedure we employ is the following. Our first step is to examine stationarity using KPSS and ADF tests. Even if these procedures indicate stationarity or no unit root, we presume that the series may contain a shift in the variance and, therefore, the complementary test should be applied. If the test statistics of complementary test is less than the critical level we conclude that the data are completely covariance stationary. Otherwise, if the null of homogeneity of unconditional variance is rejected, and we conclude that although there is no unit root the data are not covariance stationary and have shifts in variance.

The rest of the paper is organized as follows. Section 2 discusses theory of stationarity and unit root tests; Section 3 presents data; Section 4 illustrates empirical results; and Section 5 concludes.

\section{Theory}

\section{II.1 On stationarity}

A time series is stationary if the underlying rules that generate the series do not change over time. A stationary time series is easy to predict since its statistical properties are assumed to be the same in the future. In reality a time series may be stationary with respect to some characteristic (such as mean), and at the same time be non-stationary with respect to others (such as variance or correlation structure). In general, a time series without time trend, with constant mean and covariance, and with no periodic or seasonal fluctuations will be called stationary. Any 
type of seasonal fluctuation violates stationarity, since seasonal fluctuations imply that the mean, at least, shifts over time.

\section{II.2 Unit Root Tests}

\section{II.2.1 Augmented Dickey-Fuller Test}

Formal tests to determine whether a time series contains a stochastic trend are called unit root tests. The classical is the Augmented Dickey-Fuller (ADF) test. Having a process $X_{t}$, the ADF test is to find whether $\mathrm{X}_{\mathrm{t}}$ has unit root or stationary (around a level or deterministic trend) by running the following test regression:

$$
\Delta \mathrm{X}_{\mathrm{t}}=\phi(t)+\gamma \mathrm{X}_{\mathrm{t}}+\sum_{j=1}^{p} \beta_{j} \Delta X_{t-j}+\varepsilon_{t}
$$

where $\Delta \mathrm{X}_{\mathrm{t}}=\mathrm{X}_{\mathrm{t}}-\mathrm{X}_{\mathrm{t}-1}$ is a time series return; $\phi(t)$ is a deterministic level and/or time trend component; and $\varepsilon_{\mathrm{t}}$ is a stationary error term. The null hypothesis is that the process is integrated: $\mathbf{H}_{0}: \gamma=0$ (i.e. a unit root is present); while the alternative is $\mathbf{H}_{1}: \gamma<0$ (i.e. time series is stationary around deterministic component). The $p$ lagged regressors are included to decrease the nuisance caused by autocorrelation structure of the disturbances, $\varepsilon_{t}$. It is well-known (see Koehler and Murphree (1988), Hall (1994), Ng and Peron (1995)) that ADF tests are sensitive to the choice of the number of lags, p. The classical selection criteria are Akaike (1976) and Schwartz (1978). The optimal lag length for this work is selected based on the Modified Akaike Information Criterion, offered by $\mathrm{Ng}$ and Perron (2001).

\section{II.2.2 Stationarity Test (KPSS)}

The KPSS test (Kwiatkowski et al (1992)) is used to check whether observable time series is stationary around a deterministic level and/or trend against the alternative of unit root. This test is well-known to be the most powerful among unit root tests family. The KPSS test model for the examined variable, $X_{t} t=1, \ldots, T$, is: 


$$
X_{t}=\phi(t)+r_{t}+\varepsilon_{t}
$$

In this model, $\phi(t)$ is a deterministic level and/or time trend component; $r_{t}=r_{t-1}+u_{t}$ is a random walk process, and $\varepsilon_{\mathrm{t}}$ is a stationary disturbance term. The null hypothesis of stationarity implies that the random walk has zero variance $\mathbf{H}_{0}: \sigma_{u}^{2}=0$ (i.e. the process $r_{t}$ is constant). Under the level stationarity the KPSS test equation is $X_{t}=\alpha_{0}+e_{t}$. Partial sum of residuals $S_{t}=\sum_{i=1}^{t} \hat{e}_{i}$ - are used to obtain the test statistics $-K P S S=(T \sigma)^{-2} \sum_{t=1}^{T} S_{t}^{2}$, where under the null hypothesis $\sigma^{2}$ is a consistent estimator of the long run variance of the error term $\varepsilon_{\mathrm{t}}\left(\sigma_{\varepsilon}^{2}\right)$ which is defined as $\sigma^{2}=\lim _{T \rightarrow \infty} T^{-1} \mathrm{E}\left(\left[\sum_{t=1}^{T} \varepsilon_{t}\right]^{2}\right)$.

The KPSS test is useful as a confirmation test. For example, if using the ADF test one cannot reject the unit root null and using the KPSS test one can reject the stationarity null, then the data is integrated, i.e. has a unit root. On the other hand if using the ADF test it is possible to reject the unit root null, but using the KPSS test it is impossible to accept the stationarity null, then further examination is needed since tests contradict each other.

\section{II.3 Complementary Test}

Ahamada (2004) uses simulations to show some failures of the KPSS test to detect nonstationarity that can be caused by swings in unconditional variance. Because data may have time-changing unconditional variance, non-rejection of the null of stationarity by the KPSS does not necessarily imply stationarity. Liew et al (2007) show that the ADF test fails to detect unconditional variance non-stationarity. In both works, complementary test - tailored from the cumulative sum of squares procedure by Inclan and Tiao (1994) - is offered in order to detect this type of nonstationarity in the covariance structure. 
Due to the nature of the electricity price its volatility - measured by unconditional variance varies over time (for example see Knittel and Roberts (2005), Li and Flynn (2004), Hadsell et al (2004), Higgs and Worthington (2005), Hadsell and Shawky (2006), Weron (2005)). This implies that the null of homogeneity of the unconditional variance should be tested in the electricity prices against the alternative of the unconditional variance time-fluctuating.

The complementary test can be defined as following. Consider a time process $X_{t} t=1, \ldots, T$. To be sure that the data is completely stationary after non-rejection of the KPSS null and rejection of the ADF null, a variance homogeneity test must be run: $X_{t}=X_{0}+\varepsilon_{t}$, where $X_{0}$ is a fixed level. The null hypothesis is that $\varepsilon_{\mathrm{t}}$ is a zero mean stationary process with constant

variance $E\left(\varepsilon_{t}^{2}\right)=\sigma_{t}^{2}=\sigma^{2}>0$. The test statistics is defined as follows:

$$
\begin{aligned}
& \tau=\max _{k=1, \ldots, T}\left\{\sqrt{T / 2}\left|D_{k}\right|\right\}, \\
& D_{k}=\frac{C_{k}}{C_{T}}-k / T, \\
& C_{k}=\sum_{i=1}^{k} e_{i}^{2}, \quad k=1, \ldots, T
\end{aligned}
$$

Inclan and Tiao (1994) prove that under the null of variance homogeneity and assuming that $\left\{\varepsilon_{t}\right\}$ is an i.i.d process, the limiting distribution of $\tau$ is given by $\sup \left(\mathrm{W}_{t}\right)$ where $\mathrm{W}_{t}$ is a standard Brownian Bridge. The critical values are 1.628 for $1 \%, 1.358$ for $5 \%$, and 1.224 for $10 \%$ of confidence (see Inclan and Tiao (1994), Table 1).

\section{Electricity Markets: Data Description}

\section{III.1 Data}

We use market data from the PJM real-time and day-ahead forward markets. The data set consists of six years of hourly real-time spot prices and day-ahead forward prices from June 1, 2000 to May 31,2006 . The prices are quoted in dollars per megawatt hour, $(\$ / M W h)$ for each on the 24 hours for each of 2191 days (six years) in the sample. The data were retrieved from the PJM 
website www.pjm.com. Due to non-storability of electricity, the electricity prices behavior changes throughout the day; therefore the data set is divided into 24 separate time series, for which tests are applied separately.

Summary statistics for electricity spot and forward prices are provided in Table 5-1 Table 5-2. All statistics vary during a day and are higher for on-peak ${ }^{11}$ hours than for off-peak hours. For most of the hours spot prices are on average higher than forward prices, though median spot prices are lower than median forward prices. Both real-time and day-ahead prices display significant excess positive skewness (the median is less than the mean for all hours), high volatility (standard deviation is larger than unity), and fat tails (kurtosis is notably higher than 3).

\section{III.2 Seasonal Filtering}

Seasonal variations or cycles - by definition - are regularly repeating movements in series values that can be tied to recurring events. Electricity prices have strong seasonal patterns at different frequencies including diurnal, weekly, and annual. In order to avoid spurious conclusions from ADF and KPSS tests ${ }^{12}$, we apply unit root tests to the deseasoned series $X_{t}$, defined as the following:

$$
P_{\mathrm{t}}=\Delta \mathrm{t}+\sum_{i=1}^{6} \alpha_{i} W_{i}+\sum_{j=1}^{11} \beta_{j} M_{j}+\sum_{k=1}^{6} \gamma_{k} A_{k}+X_{t}+\varepsilon_{t}
$$

where $P_{t}$ is electricity prices; $t$ stands for time trend; $W_{i}$ are weekly dummies (e.g., 1 if Monday and 0 otherwise) to catch weekly cycle; $M_{j}$ are monthly dummies (e.g., 1 if January and 0 otherwise) to catch seasonal fluctuations; and $A_{i}$ are yearly dummies (e.g., 1 if 2000 , and 0 otherwise) to treat annual changes. The deseasoned series, $X_{t}$, are used for further testing.

\section{Empirical results}

\footnotetext{
${ }^{11}$ On-peak hours are $8 \mathrm{am}-11 \mathrm{pm}$, while off-peak hours are $11 \mathrm{pm}-7 \mathrm{am}$

${ }^{12}$ Enders (1995)
} 
Table 5-3 reports unit root ADF and stationarity KPSS test ${ }^{13}$ results for both real-time and day-ahead electricity prices. The results clearly show no unit root for all hours for at minimum of 95\% confidence level (ADF statistics for real-time hours of 6-10am, 8pm, and 10-11pm and 10am of day-ahead are statistically significant at 5\%). Observing test statistics of the KPSS test we can accept stationarity around level hypothesis at $10 \%$. Despite this it is worth checking if the data are covariance stationary, since both KPSS and ADF tests are not sensitive to shifts in unconditional variance. To do so we apply the complementary test, results of which are summarized in the Table 5-4.

It is clear that the null hypothesis of covariance homogeneity can be safely rejected with $99 \%$ of confidence for all hours in both real-time and day-ahead markets. One of conclusions may be drawn is that traditional methodologies requiring stationarity -- such as ARMA and ARCH/GARCH -- are not necessarily appropriate for the electricity prices modeling. Moreover, it is imperative to take into account seasonal cycles when electricity prices are studied. To illustrate this point, let's consider a traditional approach for market informational efficiency testing, which requires examining of whether market prices are random walk (i.e. have unit root) or stationary (i.e. predictable). Since both ADF and KPSS tests produce spurious results if seasonal fluctuations are left unattended, then any conclusion about the market's efficiency may be erroneous. A solution may be either to filter seasonal fluctuations out or include seasonal dummies into unit root tests regressions. Neither of these is usually done for research on electricity prices ${ }^{14}$.

\section{Conclusion}

In this paper we analyzed the problem of stationarity in electricity price series by reviewing the literature and running classical and additional tests. The growing literature on electricity prices properties and characteristics indicates that there are strong seasonal cycles in electricity prices.

\footnotetext{
${ }^{13}$ In order to avoid singularity in regression estimation for both ADF and KPSS tests, a constant of 5 was added to real-time and day-ahead de-seasoned time series.

${ }^{14}$ See Literature Review in the Introduction. Emery and Liu (2002) de-season futures electricity price
} 
Despite this fact few studies address problem associated with seasonality directly (for instance see Emery and Liu (2002)). Based on simulation results provided by Ahamada (2003) and Liew et al (2007), we argue that some conclusions drawn in existing studies of electricity market efficiency as well as real-time and day-ahead markets cointegration may be misguided due to spurious results produced by classical stationarity tests - such as ADF and KPSS.

We empirically show that de-seasoned electricity prices can be thought as being stationary based on ADF and KPSS tests. However, since non-stationarity may be due to covariance shifts and since neither ADF nor KPSS test can detect this, a complementary test is needed to detect possible variance changes over time. Such a complementary test was developed by Inclan and Tiao (1994). An application of it for the electricity real-time and day-ahead prices is given in this work. While neither ADF nor KPSS signal of non-stationarity in de-seasoned electricity prices, the complementary test indicates that unconditional variance is not homogenious over time. Therefore, the day-ahead and real-time electricity prices may not be treated as stationary, though they do not have a unit root. 
Table 5-1: Summary Statistics for Real-Time Prices

\begin{tabular}{|c|c|c|c|c|c|c|c|}
\hline Hour & Min & Median & Mean & Max & $\begin{array}{c}\text { Standard } \\
\text { Deviation }\end{array}$ & $\begin{array}{c}\text { Skewness } \\
\text { Coefficient }\end{array}$ & $\begin{array}{c}\text { Kurtosis } \\
\text { Coefficient }\end{array}$ \\
\hline $\mathbf{1}$ & 1.29 & 20.65 & 24.64 & 117.57 & 13.66 & 2.05 & 8.93 \\
\hline $\mathbf{2}$ & -19.12 & 19.16 & 23.45 & 137.51 & 14.19 & 2.41 & 12.61 \\
\hline $\mathbf{3}$ & -3.21 & 17.75 & 21.57 & 122.69 & 12.81 & 2.43 & 12.10 \\
\hline $\mathbf{4}$ & -3.27 & 17.05 & 20.88 & 144.46 & 13.06 & 2.90 & 16.59 \\
\hline $\mathbf{5}$ & 0.82 & 17.74 & 22.17 & 125.07 & 13.86 & 2.63 & 12.37 \\
\hline $\mathbf{6}$ & -19.72 & 21.99 & 27.08 & 177.22 & 17.68 & 2.50 & 12.73 \\
\hline $\mathbf{7}$ & 0.06 & 29.86 & 37.95 & 251.62 & 27.72 & 1.95 & 8.53 \\
\hline $\mathbf{8}$ & 1.45 & 32.99 & 40.42 & 218.95 & 27.78 & 1.82 & 7.28 \\
\hline $\mathbf{9}$ & 2.93 & 35.38 & 40.87 & 200.65 & 23.93 & 1.75 & 7.55 \\
\hline $\mathbf{1 0}$ & 12.57 & 39.41 & 44.30 & 222.97 & 23.80 & 1.49 & 6.39 \\
\hline $\mathbf{1 1}$ & 13.28 & 43.48 & 48.49 & 247.76 & 25.42 & 1.50 & 7.29 \\
\hline $\mathbf{1 2}$ & 12.48 & 42.53 & 47.84 & 623.01 & 30.81 & 6.08 & 93.67 \\
\hline $\mathbf{1 3}$ & 12.38 & 41.50 & 47.83 & 916.68 & 38.38 & 10.54 & 209.15 \\
\hline $\mathbf{1 4}$ & 9.42 & 42.86 & 50.38 & 931.43 & 43.61 & 9.32 & 152.95 \\
\hline $\mathbf{1 5}$ & 8.53 & 38.04 & 47.08 & 931.53 & 44.42 & 9.81 & 168.04 \\
\hline $\mathbf{1 6}$ & 10.23 & 36.74 & 46.47 & 931.93 & 45.44 & 8.95 & 136.02 \\
\hline $\mathbf{1 7}$ & 11.83 & 41.81 & 50.77 & 932.27 & 45.57 & 9.05 & 145.88 \\
\hline $\mathbf{1 8}$ & 6.23 & 47.77 & 55.81 & 932.25 & 43.20 & 8.30 & 150.87 \\
\hline $\mathbf{1 9}$ & 6.81 & 43.03 & 51.39 & 801.55 & 37.39 & 6.87 & 116.65 \\
\hline $\mathbf{2 0}$ & 5.49 & 42.03 & 49.00 & 375.45 & 30.02 & 2.15 & 12.85 \\
\hline $\mathbf{2 1}$ & 13.18 & 46.16 & 51.90 & 909.08 & 34.01 & 8.35 & 189.30 \\
\hline $\mathbf{2 2}$ & 13.40 & 40.11 & 44.95 & 325.04 & 24.79 & 1.96 & 12.83 \\
\hline $\mathbf{2 3}$ & 9.47 & 28.33 & 31.90 & 117.82 & 15.92 & 1.63 & 6.53 \\
\hline $\mathbf{2 4}$ & 0.78 & 23.08 & 27.54 & 142.08 & 14.82 & 2.07 & 9.67 \\
\hline
\end{tabular}

Note: 01 June 2000 - 31 May 2006 
Table 5-2: Summary Statistics for Day-Ahead Prices

\begin{tabular}{|c|c|c|c|c|c|c|c|}
\hline Hour & Min & Median & Mean & Max & $\begin{array}{c}\text { Standard } \\
\text { Deviation }\end{array}$ & $\begin{array}{c}\text { Skewness } \\
\text { Coefficient }\end{array}$ & $\begin{array}{c}\text { Kurtosis } \\
\text { Coefficient }\end{array}$ \\
\hline $\mathbf{1}$ & 1.63 & 22.71 & 25.46 & 94.61 & 11.67 & 1.62 & 6.66 \\
\hline $\mathbf{2}$ & 0.66 & 19.43 & 22.40 & 85.02 & 10.50 & 1.63 & 7.02 \\
\hline $\mathbf{3}$ & 0.68 & 17.90 & 20.83 & 82.45 & 10.20 & 1.57 & 7.02 \\
\hline $\mathbf{4}$ & 0.50 & 17.19 & 20.23 & 81.45 & 10.39 & 1.60 & 7.33 \\
\hline $\mathbf{5}$ & 0.10 & 17.91 & 21.17 & 87.12 & 11.03 & 1.72 & 7.79 \\
\hline $\mathbf{6}$ & 0.10 & 22.12 & 25.76 & 105.54 & 13.66 & 1.77 & 8.00 \\
\hline $\mathbf{7}$ & 0.22 & 31.22 & 36.76 & 156.70 & 22.59 & 1.56 & 6.43 \\
\hline $\mathbf{8}$ & 0.21 & 34.72 & 40.00 & 155.71 & 21.90 & 1.42 & 5.92 \\
\hline $\mathbf{9}$ & 11.01 & 37.73 & 41.46 & 153.00 & 19.44 & 1.30 & 5.83 \\
\hline $\mathbf{1 0}$ & 12.96 & 41.69 & 44.24 & 152.94 & 18.70 & 1.09 & 5.00 \\
\hline $\mathbf{1 1}$ & 13.63 & 44.84 & 46.69 & 177.68 & 19.50 & 1.23 & 6.10 \\
\hline $\mathbf{1 2}$ & 14.05 & 44.67 & 46.81 & 320.01 & 21.30 & 2.66 & 23.97 \\
\hline $\mathbf{1 3}$ & 14.43 & 42.55 & 46.20 & 384.11 & 24.00 & 3.85 & 39.57 \\
\hline $\mathbf{1 4}$ & 14.02 & 42.17 & 46.94 & 550.01 & 28.63 & 5.95 & 79.55 \\
\hline $\mathbf{1 5}$ & 13.93 & 40.34 & 46.60 & 700.00 & 33.47 & 7.76 & 118.88 \\
\hline $\mathbf{1 6}$ & 14.00 & 39.99 & 46.95 & 701.03 & 34.66 & 7.18 & 104.50 \\
\hline $\mathbf{1 7}$ & 15.04 & 43.62 & 50.03 & 650.01 & 33.46 & 6.14 & 82.54 \\
\hline $\mathbf{1 8}$ & 15.03 & 49.62 & 56.40 & 500.01 & 31.28 & 3.61 & 35.59 \\
\hline $\mathbf{1 9}$ & 14.91 & 49.75 & 55.46 & 450.01 & 27.96 & 2.66 & 25.49 \\
\hline $\mathbf{2 0}$ & 15.12 & 48.35 & 53.24 & 356.98 & 25.87 & 2.17 & 15.68 \\
\hline $\mathbf{2 1}$ & 15.10 & 48.71 & 51.95 & 296.58 & 23.29 & 1.66 & 10.57 \\
\hline $\mathbf{2 2}$ & 15.00 & 42.34 & 44.70 & 178.78 & 19.71 & 1.34 & 6.46 \\
\hline $\mathbf{2 3}$ & 12.68 & 31.53 & 33.71 & 106.70 & 14.18 & 1.30 & 5.33 \\
\hline $\mathbf{2 4}$ & 1.38 & 25.73 & 28.41 & 96.89 & 12.46 & 1.39 & 5.54 \\
\hline
\end{tabular}

Note: 01 June 2000 - 31 May 2006 
Table 5-3: ADF Level Unit Root and KPSS Level Stationarity Tests Results

\begin{tabular}{|c|c|c|c|c|}
\hline \multirow[b]{3}{*}{ Hour } & \multicolumn{2}{|c|}{ ADF } & \multicolumn{2}{|c|}{ KPSS } \\
\hline & \multicolumn{2}{|c|}{$\mathrm{H}_{0}$ : level Unit Root } & \multicolumn{2}{|c|}{$\mathrm{H}_{0}$ : level Stationarity } \\
\hline & RT & DA & RT & DA \\
\hline 1 & $-5.141^{A}$ & $-5.019^{A}$ & 0.089 & 0.096 \\
\hline 2 & $-5.255^{A}$ & $-5.027^{A}$ & 0.081 & 0.091 \\
\hline 3 & $-5.636^{A}$ & $-5.097^{A}$ & 0.077 & 0.089 \\
\hline 4 & $-5.751^{A}$ & $-5.082^{A}$ & 0.070 & 0.088 \\
\hline 5 & $-3.717^{\mathrm{A}}$ & $-5.074^{A}$ & 0.088 & 0.090 \\
\hline 6 & $-3.017^{B}$ & $-4.759^{\mathrm{A}}$ & 0.104 & 0.100 \\
\hline 7 & $-3.173^{\mathrm{B}}$ & $-3.619^{A}$ & 0.126 & 0.116 \\
\hline 8 & $-2.894^{\mathrm{B}}$ & $-3.777^{A}$ & 0.131 & 0.122 \\
\hline 9 & $-3.219^{B}$ & $-3.872^{A}$ & 0.123 & 0.121 \\
\hline 10 & $-3.239^{B}$ & $-3.397^{B}$ & 0.132 & 0.125 \\
\hline 11 & $-3.936^{A}$ & $-3.847^{A}$ & 0.124 & 0.124 \\
\hline 12 & $-3.883^{A}$ & $-3.982^{A}$ & 0.097 & 0.116 \\
\hline 13 & $-4.871^{A}$ & $-4.351^{A}$ & 0.078 & 0.100 \\
\hline 14 & $-4.932^{\mathrm{A}}$ & $-4.844^{\mathrm{A}}$ & 0.070 & 0.083 \\
\hline 15 & $-5.282^{A}$ & $-4.962^{A}$ & 0.055 & 0.068 \\
\hline 16 & $-4.883^{A}$ & $-4.857^{A}$ & 0.073 & 0.067 \\
\hline 17 & $-4.819^{A}$ & $-4.460^{A}$ & 0.081 & 0.079 \\
\hline 18 & $-4.316^{A}$ & $-4.259^{A}$ & 0.103 & 0.110 \\
\hline 19 & $-3.948^{A}$ & $-3.647^{A}$ & 0.108 & 0.124 \\
\hline 20 & $-2.924^{\mathrm{B}}$ & $-3.851^{A}$ & 0.123 & 0.120 \\
\hline 21 & $-4.063^{A}$ & $-3.944^{A}$ & 0.103 & 0.119 \\
\hline 22 & $-3.334^{\mathrm{B}}$ & $-3.899^{A}$ & 0.128 & 0.125 \\
\hline 23 & $-3.352^{B}$ & $-4.429^{A}$ & 0.121 & 0.108 \\
\hline 24 & $-5.068^{A}$ & $-5.419^{A}$ & 0.089 & 0.090 \\
\hline
\end{tabular}

Note: A -- 1\% significance; $B$--- $5 \%$ significance

Critical values for level ADF test: -3.433 at $1 \%,-2.863$ at $5 \%$, and -2.567 at $10 \%$. Critical values for level KPSS test: 0.347 at $10 \%, 0.463$ at $5 \%$, and 0.739 at $1 \%$.

Real-Time (RT) and Day-Ahead (DA) are De-seasoned Prices

01 June 2000 - 31 May 2006 
Table 5-4: Complementary Test for Stationarity for Electricity Prices

\begin{tabular}{|c|c|c|}
\hline Hour & Real-Time & Day-Ahead \\
\hline 1 & $8.210^{A}$ & $7.359^{A}$ \\
\hline 2 & $9.009^{A}$ & $8.364^{A}$ \\
\hline 3 & $8.870^{A}$ & $8.402^{\mathrm{A}}$ \\
\hline 4 & $9.626^{\mathrm{A}}$ & $8.353^{\mathrm{A}}$ \\
\hline 5 & $9.807^{\mathrm{A}}$ & $8.534^{\mathrm{A}}$ \\
\hline 6 & $9.256^{\mathrm{A}}$ & $8.209^{A}$ \\
\hline 7 & $8.411^{\mathrm{A}}$ & $5.756^{\mathrm{A}}$ \\
\hline 8 & $6.395^{\mathrm{A}}$ & $4.263^{\mathrm{A}}$ \\
\hline 9 & $6.073^{A}$ & $3.788^{\mathrm{A}}$ \\
\hline 10 & $5.077^{\mathrm{A}}$ & $3.348^{A}$ \\
\hline 11 & $4.060^{\mathrm{A}}$ & $2.564^{\mathrm{A}}$ \\
\hline 12 & $10.311^{A}$ & $7.274^{\mathrm{A}}$ \\
\hline 13 & $14.678^{A}$ & $9.857^{\mathrm{A}}$ \\
\hline 14 & $15.596^{\mathrm{A}}$ & $13.322^{A}$ \\
\hline 15 & $16.254^{\mathrm{A}}$ & $15.519^{A}$ \\
\hline 16 & $13.037^{\mathrm{A}}$ & $14.505^{\mathrm{A}}$ \\
\hline 17 & $13.408^{\mathrm{A}}$ & $12.701^{\mathrm{A}}$ \\
\hline 18 & $14.420^{\mathrm{A}}$ & $8.769^{\mathrm{A}}$ \\
\hline 19 & $11.495^{\mathrm{A}}$ & $7.199^{A}$ \\
\hline 20 & $5.211^{\mathrm{A}}$ & $5.693^{\mathrm{A}}$ \\
\hline 21 & $10.816^{\mathrm{A}}$ & $4.355^{\mathrm{A}}$ \\
\hline 22 & $3.810^{\mathrm{A}}$ & $3.014^{\mathrm{A}}$ \\
\hline 23 & $4.918^{\mathrm{A}}$ & $3.345^{\mathrm{A}}$ \\
\hline 24 & $7.336^{A}$ & $4.053^{\mathrm{A}}$ \\
\hline
\end{tabular}

Note: A -- $1 \%$ significance; $B$--- $5 \%$ significance

Critical Values are 1.224 at $10 \%, 1.358$ at $5 \%$, and 1.628 at $1 \%$. Real-Time (RT) and Day-Ahead (DA) are De-seasoned Prices 01 June 2000 - 31 May 2006 


\section{Chapter 6 : Conclusion}

This dissertation has addressed various aspects of modeling equilibrium electricity prices. Each essay examines different characteristics of electricity prices and their derivatives as well as the influence of spatial and technical attributes of transmission line system and fuel storage inventories on wholesale electricity price dynamics.

Analysis of electricity prices currently receives a great deal of attention. In Chapter 2 we provide a detailed examination of the spatial interdependence of zonal electricity prices due to the transmission lines structure. The effect of spatial correlation and its gravity is empirically estimated using spatial autocorrelation (SAR) in errors model on Locational Marginal Prices from the PJM interconnection markets. We have demonstrated that the SAR in errors model is a potentially useful tool for analysis of electricity prices in interconnected and interdependent markets. Evidence that higher transmission congestion lessens spatial ties in the electricity market is found empirically. This may be of particular interest for market participants for constructing an effective hedging position. The problem of unobserved spatial correlation in the system must be addressed in price modeling to avoid bias and inconsistency in forecasting electricity prices in any particular zone, a result that can be explored in future research.

Chapter 3 discusses the proposition that any complete model of the electricity forward premium must include information about fuel storage inventories. An empirical model of the effect of natural gas storage on the electricity forward premium built based on the model of Bessembinder and Lemmon (2002) by incorporating insights about the source of electricity price skewness and variance similar to those of Routledge, Seppi, and Spatt (2001). Because of the financial importance of electricity price distribution moments, improving our understanding of the role of gas storage inventories on electricity price skewness and variance should be of value to practitioners. Understanding the link between natural gas storage inventories and electricity market price risk may also improve our ability to understand and quantify the value of gas storage facilities, both to the 
markets and to society at large. Our empirical results confirm the results reported in Longstaff and Wang (2004), and offer a methodological improvement. Moreover, they go beyond Bessembinder and Lemmon and Longstaff and Wang by showing strong support for the proposition that the availability of stored gas has a measurable effect on the electricity forward premium, and that information about gas storage inventories adds information to electricity price analysis beyond the information contained in the temperature data by itself. Further research is required on practical applications of our model to the models currently in use by utilities, power producers, gas storage managers, and other practitioners. For practical applications to the electricity markets and to the needs of practitioners, the empirical model will require modifications depending upon the availability of information and the characteristics of demand and supply.

Chapter 4 sheds some light on the economic efficiency of electricity markets. A number of econometric tests - such as a Portmanteau white noise test, unit root tests, and multivariate variance ratio (MVR) random walk test - is used to evaluated the level of informational efficiency the PJM electricity real-time and day-ahead markets. The results are mixed and disagree with each other. Only seven hours - all of them on-peak -- in both markets can be treated as random walks according to the MVR test, which constitutes about $29 \%$. This provides evidence against the conclusions drawn in other studies about electricity markets efficiency and has a very important impact on our understanding of the markets, their designs, and general effect of deregulation. Finding some level of informational inefficiency suggests that electricity prices may be forecasted in the short-run using autoregressive time series modeling methods. In addition, we comment on misuse of unit root tests in the presence of seasonal effect following empirical simulation found in Lopes (2006), and Demetrescu and Hassler (2007). It is shown that neglecting seasonal fluctuations makes the results from ADF-type tests incredible. This may compromise some inferences from current literature on electricity markets efficiency that heavily relies on unit root tests while ignoring seasonalities. There exist several possible extensions of this work: (1) incorporating seasonal effect into unit root and stationarity tests; or (2) analyzing electricity prices after seasonal filtering. 
Stationarity of time series is required for most modeling techniques. Chapter 5 analyzes stationarity of electricity prices. Ahamada (2003), Xiao and Lima (2007), and Liew et al (2007) show that classical stationarity tests may fail to detect nonstationarity that arises due to shifts in unconditional variance. Using the PJM data, I empirically show that de-seasoned electricity prices can be thought as being stationary based on ADF and KPSS tests. However, a complementary test - developed by Inclan and Tiao (1994) - detects changes in variance over time. 


\section{Bibliography}

- Akaike, H. (1976) "Canonical correlation analysis of time series and the use of the information criterion. In System Identification: Advances and Case Studies" (eds R. K. Mehra and D. G. Laintiotis), New York: Academic:27-96.

- Anselin, L. (1988). Spatial Econometrics: Methods and Models. Dordrecht: Kluwer Academic Publishers.

- Anselin, L. (2002). "Under the Hood: Issues in the Specification and Interpretation of Spatial Regression Models." Agricultural Economics: 27(3): 247-267.

- Anselin, Luc, and Rosina Moreno (2003). "Properties of tests for spatial error components" Regional Science and Urban Economics 33(5): 595-618.

- Anselin, L. (2003). "Spatial Externalities, Spatial Multipliers, and Spatial Econometrics," International Regional Science Review 26(2): 153-166.

- Arciniegas, I., Barret, C., and A. Marathe (2003). "Assessing the Efficiency of US Electricity Markets" Utilities Policy, 11: 75-86

- Baltagi, Badi and Dong Li (2001). "Double Length Artificial Regressions For Testing Spatial Dependence." Econometric Reviews 20(1): 31-40.

- Bessembinder, H., and M. Lemmon (2002), "Equilibrium Pricing and Optimal Hedging in Electricity Forward Markets," Journal of Finance 57(3), 1347-82.

- Bhanot, K. (2000). "Behavior of Power Prices: Implication for the Valuation and Hedging of Financial Contracts." Journal of Risk, 2(3): 43-62

- Bohn, R. E., Caramanis, M.C., and . C. Schweppe (1984). "Optimal Pricing in Electricity Networks over Space and Time." The RAND Journal of Economics 15(3), 360-376.

- Borenstein, S., Bushnell, J., Knittel, C., and C. Wolfram (2001). Trading Inefficiencies in California's Electricity Markets. UCEI, Working Paper No. PWP-086.

- Borenstein, S. (2002), "The Trouble With Electricity Markets: Understanding California's Restructuring Disaster," Journal of Economic Perspectives 16(1): 191-211,

- Borenstein, S, Bushnell, J., and F. Wolak (2002), "Measuring Market Inefficiencies in California's Restructured Wholesale Electricity Market," Center for the Study of Energy Markets, Working paper 102, June,

- Borenstein, S, Bushnell, J., and S. Stoft (2000), "The Competitive Effects of Transmission Capacity in a Deregulated Electricity Industry," RAND Journal of Economics 31 (summer):294-325,

- Borenstein, S., Busse, M. and R. Kellogg (2007), "Security of Supply and Forward Price Premia: Evidence from the Natural Gas Industry." Working paper, University of California. http://imio.haas.berkeley.edu/borenstein-busse-kellog030107-app.pdf

- Box, G.E.P., and D.A. Pierce (1970). "Distribution of residual autocorrelations in autoregressive-integrated moving average time series models." Journal of American Statistical Association, 65: 1509-26.

- Bunn, D. (2004). "Modeling Prices in Competitive Electricity Markets." John Wiley \& Sons, West Sussex, England

- Campbell, J.Y., Lo A.W., and A.C. MacKinlay, (1997): The Econometrics of Financial Markets. Princeton Univ. Press: Princeton.

- Campbell, J. Y., and P. Perron, (1991). "Pitfalls and Opportunities: What Macroeconomists Should Know About Unit Roots," NBER Working Papers 0100

- Chow, K.V., and K.C. Denning (1993). "A Simple Multiple Variance Ratio Test". Journal of Econometrics, 58: 385-401

- Cliff, A. D. and J. K. Ord (1973). Spatial Autocorrelation. London: Pion.

- Demetrescu, M. and U. Hassler (2007). "Effect of Neglecting Deterministic Seasonality on Unit Root Tests" Statistical Papers, 48: 385-402 
- Den Haan, W.J. and A. Levin (1997)."A Practitioner's Guide to Robust Covariance Matrix Estimation", IN: Handbook of Statistics 15: Robust Inference, Editors: Maddala, G.S., and Rao, C.R., Chapter 12, 291-341, Amsterdam, North-Holland, Elsevier

- Deng S.J. (1999). "Stochastic Models of Energy Commodity Prices and Their Applications: Mean-reversion with Jumps and Spikes," Working Paper, University of California at Berkeley

- De Vany, A.S., and W.D. Walls (1999a). "Price Dynamics in a Network of Decentralized Power Markets". Journal of Regulatory Economics, 15:123-140.

- De Vany, A.S., and W.D. Walls (1999b). "Co-integration Analysis of Spot Electricity Prices: Insights on Transmission Efficiency in the Western US." Energy Economics, 21:453-448.

- Dickey, D. A. and W. A. Fuller (1979). Distribution of the Estimators for Autoregressive Time Series with a Unit Root. Journal of the American Statistical Association, 74,427-431.

- Dickey, D. and W.A. Fuller (1981). "Likelihood Ratio Statistics for Autoregressive Time Series with a Unit Root," Econometrica, 49, 1057-1072.

- $\quad$ Dickey, D.A., Bell, W.R, and R.B. Miller (1986). "Unit Roots in Time Series Models: Tests

- Dubin, R. (1988). "Estimation of Regression Coefficients in the Presence of Spatially Autocorrelated Errors." Review of Economics and Statistics 70(3): 466-474

- Elliot, G., Rothenberg, T.J., and J.H. Stock (1996). "Efficient Tests for an Autoregressive Unit Root," Econometrica, 64, 813-836.

- Emery. G.W. and Q. Liu (2002). "An Analysis of the Relationship between Electricity and Natural Gas Futures Prices" The Journal of Futures Markets, 22(2): 95-122

- Enders, W. (1995). "Applied Econometric Time Series". New-York Wiley

- Engle, R.F., and C.W.J. Granger. (1987). "Co-integration and Error Correction: Representation, Estimation, and Testing." Econometrica, 55: 251-276.

- Engel, R. F., Granger, C.W.J., Rice, J., and A. Weiss (1986). "Semiparametric Estimates of the Relationship Between Weather and Electricity," Journal of the American Statistical Association 81(394), 310-320

- Energy Information Administration (EIA 2004), "The Basics of Underground Natural Gas Storage," last updated August 2004, available at http://www.eia.doe.gov.

- Energy Information Administration (EIA 2007a), "Annual Energy Review 2006," Report No. DOE/EIA-0384, updated June 27, 2007, available at http://www.eia.doe.gov.

- Energy Information Administration (EIA 2007b) "About Natural Gas Pipelines Transporting Natural Gas." June, available at http://www.eia.doe.gov.

- Fama, E.F., (1970) "Efficient Capital Markets: A Review of Theory and Empirical Work," Journal of Finance, 25: 383-417.

- Fama, E.F., (1990) “Efficient Capital Markets: II," Journal of Finance 46, 1575-1618.

- Fay, D., Ringwood, J., Condon, M. and M. Kelly (2003), "24-Hour Electrical load data - a sequential or parallel time series?" Neurocomputing 55 (October), 469-498.

- Feng W., Liu, S., Lai, M., and X. Deng (2007). "Empirical Research on Price Discovery Efficiency in Electricity Futures Market" Power Engineering Society General Meeting, IEEE: 1932-5517

- Ferreira, P., Soares, I., and M. Araujo (2005). "Liberalization, Consumption Heterogeneity and the Dynamics of Energy Prices". Energy Policy, 33:2244-2255

- Fuller, W. (1996). Introduction to Statistical Time Series, Second Edition. John Wiley, New York.

- Giacomini, R., and C.W.J. Granger (2004). "Aggregation of Space-time Process." Journal of Econometrics, 118: 7-26.

- Ghysels E., Lee, H.S., and J. Noh (1994). "Testing for Unit Root is Seasonal Time Series". Journal of Econometrics, 62: 415-442

- Gjolberg, O. (2001). "When (and How) Will the Markets for Oil and Electricity Become Integrated? Econometric Evidence and Trends 1993-99" ttp://www.umb.no/ios/Publikasjoner/d2001/d2001-20.PDF 
- Goto, M. and G. A. Karolyi (2004) "Understanding Electricity Price Volatility Within and Across Markets" Dice Center Working Paper No. 2004-12

- Hadsell, L., Marthe, A., and H.A. Shawky (2004) "Estimating the Volatility Of Wholesale Electricity Spot Prices in the US." The Energy Journal 25(4): 23-40.

- Hadsell, L. and H.A. Shawky (2006). "Electricity Price Volatility and the Marginal Cost of Congestion: An Empirical Study of Peak Hours on the NYISO Market, 2001-2004." The Energy Journal 27(2): 157-179.

- Haesun, P., Mjelde, J.W., and D.A. Bessler (2006). "Price Dynamics among US Electricity Spot Markets." Energy Economics, 28: 81-101

- Hall, A., (1994). "Testing for a unit root in time series with pretest data-based model selection." Journal of Business and Economic Statistics 12 (4): 461-470.

- Higgs, H., and A. Worthignton (2003). "evaluating the Informational Efficiency of Australian Electricity Spot Markets: Multiple Variance Ration Tests of Random Walks" Pacific and Asian Journal of Energy, 13(1): 1-16

- Huisamn, R., and R. Mahieu (2003). "Regime Jumps in Electricity Prices." Energy Economics, 25: 425-434

- Hogan,W. W. (1992). "Contract Networks for Electricity Power Transmission." Journal of Regulatory Economics 4(3): 211-242.

- Hogan, William W. (1993). "Markets in Real Electric Networks Require Reactive Prices." The Energy Journal 14(3): 171-200.

- Inclan, C. and G. Tiao (1994). "Use of Cumulative Sums of Squares for Retrospective Detection of Changes of Variance". Journal of American Statistical Association, 89(427): 913-923

- Jerko, C.A., Mjelde, J.W., and D.A. Bessler (2004). "Identifying Dynamic Interactions in Western US Spot Market" in Modeling Prices in Competitive Electricity Markets edited by Bunn(2004): 193-216

- Joskow PL and Kahn E. (2001) "A quantitative analysis of pricing behavior in California's wholesale electricity market during summer 2000" NBER, Working Paper 8157

- Joskow, P.L., and R. Schmalensee (1983). Markets for Power. An Analysis of Electric Utility Deregulation. Cambridge, Massachusetts, London, England: The MIT Press

- Kapoor, M. Kelejian, HH., and I. R. Prucha (2007), "Panel data models with spatially correlated error components" Journal of Econometrics, 140(1): 97-130

- Kaldor, N. (1939), "Speculation and Economic Stability," Review of Economic Studies 7, 127.

- Kelejian, H., Prucha, I.R.. (1999) "A Generalized Moments Estimator for the Autoregressive Parameter in a Spatial Model." International Economic Review 40: 509533.

- Knittel, C.R., and Roberts, M. (2005). "An Empirical Examination of Deregulated Electricity Prices." Energy Economics, 27: 791-817.

- Koehler; A.B., and E.S. Murphree (1988) "A Comparison of the Akaike and Schwarz Criteria for Selecting Model Order" Applied Statistics, 37 (2): 187-195

- Kwiatkowski, D., P.C.B. Phillips, P. Schmidt and Y. Shin (1992). "Testing the Null Hypothesis of Stationarity against the Alternative of a Unit Root," Journal of Econometrics, 54: 159-178.

- Lai, K.S., and M. Lai (1991). "A Co-integration Test for Market Efficiency." The Journal of Futures Markets, 11(5): 567-575.

- LeSage, J. P. (1999). Spatial Econometrics. Monograph, available online at the Web Book of Regional Science, http://www.rri.wvu.edu/WebBook/LeSage/spatial/spatial.html, Regional Research Institute, West Virginia University. Accompanying Matlab programs available at http://www.rri.wvu.edu/WebBook/LeSage/etoolbox/index.html

- LeSage, J. P., and R. K. Pace, eds. (2004). Advances in Econometrics, Volume 18: Spatial and Spatiotemporal Econometrics. Oxford: Elsevier. 
- LeSage, J. P., and R. K. Pace (2007), "A Matrix Exponential Spatial Specification." Journal of Econometrics, 140(1): 190-214

- Li, Y. and P.C. Flynn (2004). "Deregulated Power Prices: Comparison of Diurnal Patterns" Energy Policy, 32: 657-672

- $\quad$ Liew, V.K.S, Lau, S.H., and S.E. Ling (2007). "A Complementary Test for ADF Test with an Application to the Exchange Rates of Return". Munich Personal ReRec Archive paper N518.

- Ljung, G. M. and G.E.P. Box (1978) "On a Measure of Lack of Fit in Time Series Models," Biometrika, 65: 297-303.

- Lo, A.W. and A.C. MacKinlay (1988), "Stock Market Do Not Follow Random Walks: Evidence from a Simple Specification Test" Review of Financial Studies, 1(1): 41-66

- Lo, K.L. and Y.K. Wu (2004). "Analysis of Relationships Between Hourly Electricity Price And Load In Deregulated Real-Time Power Markets." Generation, Transmission and Distribution, IEEE Proceedings 151(4): 441-452.

- Lu, Z., and A. Y. Dong (2005). "Electricity Future Market Efficiency Testing: the Characteristics of Electricity Prices" Power Engineering Conference. The 7th International Volume, 2: 868-873

- Longstaff, F.A., and A.W. Wang (2004). "Electricity Forward Prices: A High-Frequency Empirical Analysis". Journal of Finance 59(4): 1877-1901.

- Lopes, A.C.B. da S.(2006). "Deterministic Seasonlaity in Dickey-Fuller Tests: Should WE Care?" Empirical Economics, 31: 165-182

- Lucia, J., and E.S. Schwartz (2002). "Electricity Prices and Power Derivatives: evidence from Nordic Power Exchange." Review of Derivatives Research, 5: 5-50

- MacKie-Mason J.K. (1995) "A Spatial "Smart Market" for Electric Power and Transmission" http://citeseer.ist.psu.edu/mackie-mason95spatial.html

- National Petroleum Council (NPC 2003), "Balancing Natural Gas Policy: Fueling the Demands of a Growing Economy." NPC Committee on Natural Gas, report requested by US Secretary of Energy. http://www.npc.org/reports/ng.html

- Ng, S., P. Perron (1995). "Unit root tests in ARMA models with data-dependent methods for the selection of the truncation lag." Journal American Statistical Association, 90 (429): 268-281.

- Ng, S. and P. Perron (2001). "Lag Length Selection and the Construction of Unit Root Tests with Good Size and Power." Econometrica, 69:1519-1554

- Ramanathan, R., R. Engel, C. W. J. Granger, and F. V. Araghi (1997), "Short-Run Forecast of Electricity Load and Peaks". International Journal of Forecasting 13, 161-174

- Roberts, H., (1967): "Statistical versus Clinical Prediction of the Stock Market," Unpublished manuscript, CRSP, University of Chicago.

- Ord, J. Keith (1975). "Estimation Methods for Models of Spatial Interaction." Journal of the American Statistical Association, 70:120-126.

- Paelinck, J. H. P. and L. H. Klaassen (1979). Spatial Econometrics. Saxon House, Farnborough.

- Park, H., J. W. Mjelde, and D. A. Bessler (2006) "Price Dynamics Among U.S. Electricity Spot Markets." Energy Economics 28: 81-101.

- Phillips, P.C.B., and S. Jin (2002). "The KPSS Test with Seasonal Dummies". Economic Letters, 77: 239-243

- $\quad$ Routledge, S., D.J. Seppi, and C.S. Spatt (2000), "Equilibrium Forward Curves for Commodities," Journal of Finance 55, 1297-338

- Routledge, S., D., J. Seppi, and C.S. Spatt (2001). "The "Spark Spread": An Equilibrium Model of Cross-Commodity Price Relationship in Electricity". Working paper, Carnegie Mellon University.

- Samuelson, P., (1965): "Proof that Properly Anticipated Prices Fluctuate Randomly," Industrial Management Review, 6: 41-49 
- Shawky, H.A., Marathe, A., and C, L. Barrett (2003). "A First Look at the Empirical Relation between Spot and Futures Electricity Prices in the United States." The Journal of Futures Markets, 23(10): 931-955

- Schwarz, G. (1978) "Estimating the dimension of a model". Ann. Statist., 6: 461464.

- Ullrich, C. J. (2006). "Constrained Capacity and Equilibrium Forward Premia in Electricity Markets." Working Paper, Pennsylvania State University. Available at SSRN: http://ssrn.com/abstract=923082 .

- Von der Fehr, N.H. and D. Harbord (1998) "Competition in Electricity Spot Markets. Economic Theory and International Experience," Memorandum 05/1998, Oslo University, Department of Economics.

- Weron, R. (2005). "Heavy Tails and Electricity Prices". The Deutsche Bundesbank's Annual Conference Proceedings

- Weron, R. (2006). "Modeling and Forecasting Electricity Loads and Prices: A Statistical Approach" The Wiley Finance Series

- Weron, R., Bierbrauer, M., and S. Truck (2004). "Modeling Electricity Prices: Jump Diffusion and Regime Switching." Physica A, 336: 39-48

- Weron, R., and B. Przybylowicz (2000). "Hurst Analysis of Electricity price Dynamics" Physica A, 283: 462-268

- Working, H. (1948), Theory of the inverse carrying charge in futures markets, Journal of Farm Economics 30, 1-28.

- Worthington, A., and H. Higgs (2004). "The Relationship Between Energy Spot and Futures Prices: Evidence from the Australian Electricity Market" ICFAL Journal of Applied Economics, 3(4):65-82

- Worthington, A., Kay-Spratley, A., and H. Higgs (2005). "Transmission of Prices and Price Volatility in Australian Electricity Spot Markets: A Multivariate GARCH Analysis." Energy Economics 27(2): 337-350.

- Wolfram, C., (1999), "Measuring Duopoly in the British Electricity Spot Market", American Economic Reviews, 89(4): 805-826.

- Xiao Z., and L.R. Lima (2007). "Testing Covariance Stationarity." Econometric Reviews, 26(6): 643-667 


\section{Curriculum Vitae JULIA N. POPOVA}

College of Business and Economics West Virginia University, P.O. Box 6025 Morgantown, WV 26505-6025
304-293-7964 (office); 304-906-7760 (cell)

jnpopova@mail.wvu.edu

http://www.be.wvu.edu/divecon/econ/jpopova

\section{Education}

PhD, Economics, West Virginia University, August 2008

M.A, Economics, CERGE_El, Czech Republic, August 2003

M.S., Mathematics, Novosibirsk State University, Russia, June 2001

\section{Professional Experience:}

Instructor: Department of Economics, WVU (2004-2008)

- Principles of Microeconomics (in-room, on-line)

- Principles of Macroeconomics (in-room, on-line)

- Survey of Finance and Personal Finance (in-room, on-line)

- Business Finance (in-room)

Research Assistant: West Virginia University, USA (2003 - 2005)

Teaching Assistant: CERGE-EI, Czech Republic (2001-2003)

- Mathematics for Economists and Graduate Macroeconomics

\section{Research}

o "Storage and the Electricity Forward Premium," (with Stratford Douglas), Energy Economics, 30(4):1712-1727, 2008

o "Econometric Estimation of Spatial Patterns in Electricity Prices," (with Stratford Douglas), resubmitted, The Energy Journal

o "Do Electricity Prices Follow Random Walk"

o "Note on Stationarity of Electricity Prices"

o "Analysis of Electricity Contracts Using the Principle Components Method"

\section{Honors and Awards}

o 2004 Best Student Paper Award US Association for Energy Economics

o 2005-2007 Dadisman Supplement Award, WVU

o 2004 Kaiser Best Doctoral Student Award, WVU

o 2003-2006 Swiger Supplement Award, WVU

o 2002- 2003 Phillip Morris Scholarships Award, CERGE-EI

\section{Conferences:}
o Midwest Finance Association Annual Meeting, 2007
o Southern Economic Association Annual Meeting 2006
- USAEE/IAEE North American Conference 2004, 2005, 2006
o 25th Annual Eastern Conference 2006
o 6th Annual Missouri Economics Conference 2006
o DOE/NSF EPSCoR Conference 2005

\section{Other Professional Experience: Referee: The Energy Journal}

